nlin.SI/0102010

\title{
Symmetries of Supersymmetric Integrable Hierarchies of KP Type
}

\author{
E. Nissimov ${ }^{1}$ and S. Pacheva ${ }^{1}$ \\ Institute for Nuclear Research and Nuclear Energy, Bulgarian Academy of Sciences \\ Boul. Tsarigradsko Chausee 72, BG-1784 Sofia, Bulgaria j
}

\begin{abstract}
This paper is devoted to the systematic study of additional (non-isospectral) symmetries of constrained (reduced) supersymmetric integrable hierarchies of KP type - the so called $S K P_{\left(R ; M_{B}, M_{F}\right)}$ models. The latter are supersymmetric extensions of ordinary constrained KP hierarchies which contain as special cases basic integrable systems such as (m)KdV, AKNS, FordyKulish, Yajima-Oikawa etc.. As a first main result it is shown that any $\operatorname{SKP}_{\left(R ; M_{B}, M_{F}\right)}$ hierarchy possesses two different mutually (anti-)commuting types of superloop superalgebra additional symmetries corresponding to the positive-grade and negative-grade parts of certain superloop superalgebras. The second main result is the systematic construction of the full algebra of additional Virasoro symmetries of $\operatorname{SKP}_{\left(R ; M_{B}, M_{F}\right)}$ hierarchies, which requires non-trivial modifications of the Virasoro flows known from the general case of unconstrained Manin-Radul super-KP hierarchies (the latter flows do not define symmetries for constrained $S K P_{\left(R ; M_{B}, M_{F}\right)}$ hierarchies). As a third main result we provide systematic construction of the supersymmetric analogues of multi-component (matrix) KP hierarchies and show that the latter contain among others the supersymmetric version of Davey-Stewartson system. Finally, we present an explicit derivation of the general Darboux-Bäcklund solutions for the $S K P_{\left(R ; M_{B}, M_{F}\right)}$ super-tau functions (supersymmetric "soliton"-like solutions) which preserve the additional (non-isospectral) symmetries.
\end{abstract}

PACS numbers 05.45.Y, 11.30.Pb

\footnotetext{
${ }^{1}$ E-mail: nissimov@inrne.bas.bg , svetlana@inrne.bas.bg
} 


\section{Introduction}

Supersymmetric generalization of integrable hierarchies of nonlinear evolution ( "soliton" or "solitonlike") equations is an actively developing subject whose main motivations come both from theoretical physics as well as mathematics. In theoretical physics supersymmetry is a fundamental symmetry principle unifying bosonic and fermionic degrees of freedom of infinite-dimensional dynamical (field-theoretic) systems which underly modern superstring theory as an ultimate candidate for an unified theory of all fundamental forces in Nature, including quantum gravity. In particular, supersymmetric generalizations of Kadomtsev-Petviashvili (KP) integrable hierarchy have been found [1, 2] to be of direct relevance for (multi-)matrix models of non-perturbative superstring theory. Historically, the first supersymmetric integrable system, which appeared in the literature, is the supersymmetric generalization of the Sine-Gordon equation [3]. Subsequently, the subject of supersymmetrization of KP hierarchy [4, 5, 6, 7] and other basic integrable systems (Korteveg-de Vries, nonlinear Schrödinger, Toda lattice etc.) [8, 9, 10, 11, 12, 13, 14, 15, 16] attracted a lot of interest from purely mathematical point of view, especially, the supersymmetric generalizations of the inverse scattering method, bi-Hamiltonian structures, tau-functions and Sato Grassmannian approach.

An important role in the theory of integrable systems is being played by the notion of additional (non-isospectral) symmetries whose systematic study started with the papers [17, 18]. For detailed reviews of the latter subject we refer to [19]; see also refs. 20, 21] for a systematic discussion of additional symmetries in the context of specific integrable models. Additional (non-isospectral) symmetries, by definition, consist of the set of all flows on the space of the Lax operators of the pertinent integrable hierarchy which commute with the ordinary isospectral flows, the latter being generated by the complete set of commuting integrals of motion. As shown in refs. 22] (see also [23]), there exists an equivalent definition of additional symmetries as vector fields acting on the space of $\tau$-functions (Sato Grassmannian) of the corresponding integrable hierarchy. This latter formulation allows to provide a simple interpretation of the crucial Virasoro (and $\mathbf{W}_{\mathbf{1}+\infty}$ ) constraints on partition functions of (multi-)matrix models of string theory as invariance of the $\tau$ functions (i.e., the string partition functions) under the Borel subalgebra of the Virasoro algebra of additional non-isospectral symmetries in the underlying integrable hierarchies of generalized $S L(r)$ Korteveg-de Vries (KdV) type (similarly for the $\mathbf{W}_{\mathbf{1}+\infty}$ constraints).

Recently, a deep relationship has been uncovered in ref.[24] between additional symmetries of KP hierarchy and fermionic representations of certain basic $q$-deformed ( $q$ " staying for "quantum group") hypergeometric functions playing the role of correlation functions of quantized integrable field theory models. Furthermore, the notion of additional (non-isospectral) symmetries allows to:

- Provide an alternative formulation of multi-component (matrix) KP hierarchies [28] as ordinary one-component (scalar) KP hierarchy supplemented with appropiate sets of mutually commuting additional symmetry flows (see refs. 225, 26]);

- Provide an alternative formulation of various physically relevant nonlinear evolution equations in two- and higher-dimensional space-time as additional-symmetry flows on ordinary (reduced) KP hierarchies, the most interesting examples being Davey-Stewartson and $N$-wave resonant systems (see refs.[25, 26, 27, 29]), as well as Wess-Zumino-Novikov-Witten models of group-coset-valued fields (see refs. [29, 30]) describing various ground states in string theory.

The main advantage of the above mentioned reformulation over the standard matrix pseudodifferential formulation of multi-component KP hierarchies (and their reductions) lies in the fact 
that the new formulation allows to employ the standard Darboux-Bäcklund techniques in ordinary scalar (constrained) KP hierarchies in order to generate soliton-like solutions for the more complicated multi-component (matrix) KP hierarchies [29].

The principal object of the present work is the important class of constrained (reduced) $N=1$ supersymmetric KP hierarchies introduced in ref. [14] and called " $S K P_{\left(R ; M_{B}, M_{F}\right)}$ models" (see Eq.(24) below). The latter are supersymmetric generalizations of the class $c K P_{R, M}$, called "constrained KP models", of reductions of ordinary ("bosonic") KP hierarchy containing among themselves a series of well-known integrable hierarchies such as (modified) KdV, AKNS, Fordy-Kulish, Yajima-Oikawa etc. Ð, which are collectively described by ordinary ("bosonic") Sato pseudodifferential Lax operators of the form:

$$
\mathcal{L} \equiv \mathcal{L}_{R, M}=\partial^{R}+\sum_{i=0}^{R-2} u_{i} \partial^{i}+\sum_{j=1}^{M} \Phi_{j} \partial^{-1} \Psi_{j}
$$

We will work within the framework of Sato super-pseudo-differential calculus in $N=1 \mathrm{su}-$ perspace [4, 5] (cf. Section 2 below). For alternative treatment of supersymmetric integrable hierarchies within the framework of the superspace generalization of Drinfeld-Sokolov Lie-algebraic scheme we refer to [35], see also [13, 36]. Our main task will be the systematic derivation of the full algebra of additional non-isospectral symmetries for $\operatorname{SKP}_{\left(R ; M_{B}, M_{F}\right)}$ hierarchies. Our present approach is based on a superspace extension of the approach employed in refs. [27, 29] where the full algebra of symmetries of the above mentioned ordinary bosonic constrained KP hierarchies $\mathrm{cKP}_{R, M}$ (11) has been explicitly constructed. The latter turns out to be a semi-direct product of Virasoro algebra (see also refs. 21]) with the loop algebra $(\widehat{U}(1) \oplus \widehat{S L}(M))_{+} \oplus(\widehat{S L}(M+R))_{-}$ where the subscripts $( \pm)$ indicate taking the positive/negative-grade parts of the corresponding loop algebras and the factor $(\widehat{U}(1))_{+}$corresponds to the usual isospectral flows. For the supersymmetric constrained KP hierarchies $\operatorname{SKP}_{\left(R ; M_{B}, M_{F}\right)}$ we find the full symmetry algebra, i.e., the algebra of Manin-Radul isospectral flows together with the additional non-isospectral symmetries to be a semi-direct product of Virasoro algebra with more complicated superloop superalgebras (with half-integer loop grading) of the form given in (70)-(72) and (107) below:

$$
\left(\widehat{G L}\left(M_{B}, M_{F}\right)\right)_{+} \oplus\left(\widehat{G L}^{\prime}(N+r+1, N+r+1)\right)_{-}
$$

for $\operatorname{SKP}_{\left(R ; M_{B}, M_{F}\right)}$ hierarchies which are defined by fermionic super-Lax operators with $R \equiv 2 r+1$, $M_{B}+M_{F} \equiv 2 N+1$, and:

$$
\left(\widehat{G L}_{M_{B}, M_{F}}\right)_{+} \oplus\left(\widehat{G L}_{N+r, N+r}^{\prime}\right)_{-}
$$

for $\operatorname{SKP}_{\left(R ; M_{B}, M_{F}\right)}$ hierarchies defined via bosonic super-Lax operators where $R \equiv 2 r, M_{B}+M_{F} \equiv$ $2 N$. Here again the subscripts $( \pm)$ indicate taking the positive/negative-grade parts of the corresponding superloop superalgebras, whereas the primes in (2)-(3) indicate factoring out the unit super-matrices in the pertinent integer-grade subspaces.

Let stress at this point that the superloop superalgebras $\widehat{G L}\left(N_{1}, N_{2}\right)$ (see (70)-(72) below) appearing in (2) and in what follows, are more general objects than the notion of superloop algebras

\footnotetext{
${ }^{1}$ Originally $\mathrm{cKP}_{R, M}$ hierarchies appeared in different disguises from various parallel developments: (i) symmetry reductions of the general unconstrained KP hierarchy [31, 32]; (ii) free-field realizations, in terms of finite number of fields, of both compatible first and second KP Hamiltonian structures [33]; (iii) a method of extracting continuum integrable hierarchies from generalized Toda-like lattice hierarchies underlying (multi-)matrix models in string theory $[34]$.
} 
introduced in ref. 37] in that the former possess one more Grassmannian grading. Also, let us point out that similarly to the ordinary "bosonic" case [21, 27], the construction of consistent additional Virasoro symmetries for $\operatorname{SKP}_{\left(R ; M_{B}, M_{F}\right)}$ hierarchies requires nontrivial modification of the straightforward superspace extension [2, 38] of the well-known Orlov-Schulman additional symmetry flows [18] for the general unconstrained (supersymmetric) KP hierarchy. The latter flows do not define symmetries for constrained (supersymmetric) KP hierarchies, since they do not preserve the constrained form of the pertinent (super-)Lax operators (see Section 9 below).

The plan of exposition in the present paper is as follows. In Section 2 we briefly recapitulate the main ingredients of the super-pseudo-differential operator formulation of the general unconstrained Manin-Radul $N=1$ supersymmetric KP hierarchy, including the superspace extension of such basic objects as (adjoint) super-eigenfunctions and supersymmetric squared eigenfunction potentials. In Section 3 we first briefly recall the main properties of the class $S K P_{\left(R ; M_{B}, M_{F}\right)}$ of reductions of the original unconstrained Manin-Radul super-KP hierarchy. In particular, we recall the nontrivial modification of the original Manin-Radul fermionic isospectral flows which is required for consistency of the latter with the constrained form of the pertinent super-Lax operators defining $\operatorname{SKP}_{\left(R ; M_{B}, M_{F}\right)}$ hierarchies. Also in Section 3 we present briefly the superspace extension of the pseudo-differential treatment [42] of inverse powers of (super-)Lax operators. In Section 4 we describe briefly the general formalism for studying of additional non-isospectral symmetries of supersymmetric integrable hierarchies which is the superspace extension of the formalism proposed in the first ref. 22] in the purely "bosonic" case.

The main results of the paper are contained in Sections $5-10$. In Section 5 we provide the explicit construction of positive-grade part of the superloop superalgebra of additional symmetries for constrained super-KP hierarchies defined by fermionic super-Lax operators, and the same construction is done in Section 6 for the case of constrained super-KP hierarchies defined by bosonic super-Lax operators. Section 7 presents the construction of supersymmetric analogues of multi-component (matrix) KP hierarchies out of one-component (scalar) $S K P_{\left(R ; M_{B}, M_{F}\right)}$ hierarchies by adding to the latter of an infinite subset of additional symmetry flows spanning Manin-Radul flow algebra. In particular, we find the supersymmetric extension of Davey-Stewartson system which is contained as superspace additional symmetry flow within $\operatorname{SKP}_{\left(R ; M_{B}, M_{F}\right)}$ hierarchies. Section 8 is devoted to the construction of the negative-grade part of the superloop superalgebra of additional symmetries for constrained super-KP hierarchies. In Section 9 we provide the correct Virasoro algebra of aditional symmetries for constrained $S_{K P}\left(R ; M_{B}, M_{F}\right)$ hierarchies via a non-trivial modification of the naive superspace generalization of Orlov-Schulman additional symmetry flows for ordinary "bosonic" KP hierarchy. Finally, in Section 10 we identify the explicit form of Darboux-Bäcklund transformations for constrained super-KP hierarchies which produce solutions to the same hierarchies, i.e., which are auto-Darboux-Bäcklund transformations. Further we construct the general supersymmetric Darboux-Bäcklund solutions for the superspace tau-functions of $\operatorname{SKP}_{\left(R ; M_{B}, M_{F}\right)}$ hierarchies with the additional property of preserving the additional non-isospectral symmetries. These solutions are the superspace analogues of the soliton-like solutions in the ordinary "bosonic" case.

\section{Brief Account of the General Manin-Radul Supersymmetric KP Hierarchy}

We shall use throughout the super-pseudo-differential calculus [4] as in ref. [14] with the following notations: $\partial \equiv \frac{\partial}{\partial x}$ and $\mathcal{D}=\frac{\partial}{\partial \theta}+\theta \frac{\partial}{\partial x}$ denote operators, whereas the symbols $\partial_{x}$ and $\mathcal{D}_{\theta}$ will indicate application of the corresponding operators on superfield functions. As usual, $(x, \theta)$ denote $N=1$ superspace coordinates and $\mathcal{D}^{2}=\partial$. For any super-pseudo-differential operator $\mathcal{A}=\sum_{j} a_{j / 2} \mathcal{D}^{j}$ the subscripts $( \pm)$ denote its purely differential part $\left(\mathcal{A}_{+}=\sum_{j \geq 0} a_{j / 2} \mathcal{D}^{j}\right)$ or its purely pseudo- 
differential part $\left(\mathcal{A}_{-}=\sum_{j \geq 1} a_{-j / 2} \mathcal{D}^{-j}\right)$, respectively. For any $\mathcal{A}$ the super-residuum is defined as $\operatorname{Res} \mathcal{A}=a_{-\frac{1}{2}}$. The rules of conjugation within the super-pseudo-differential formalism are as follows [12]: $(\mathcal{A B})^{*}=(-1)^{|A||B|} \mathcal{B}^{*} \mathcal{A}^{*}$ for any two elements with parities $|A|$ and $|B| ;\left(\partial^{k}\right)^{*}=$ $(-1)^{k} \partial^{k},\left(\mathcal{D}^{k}\right)^{*}=(-1)^{k(k+1) / 2} \mathcal{D}^{k}$ and $u^{*}=u$ for any coefficient superfield. Finally, in order to avoid confusion we shall also employ the following notations: for any super-(pseudo-)differential operator $\mathcal{A}$ and a superfield function $f$, the symbol $\mathcal{A}(f)$ will indicate application (action) of $\mathcal{A}$ on $f$, whereas the symbol $\mathcal{A} f$ will denote just operator product of $\mathcal{A}$ with the zero-order (multiplication) operator $f$.

The general unconstrained Manin-Radul $N=1$ supersymmetric KP (MR-SKP) hierarchy [⿶凵⿴囗十 given by a fermionic superspace Lax operator $\mathcal{L}$ :

$$
\mathcal{L}=\mathcal{D}+f_{0}+\sum_{j=1}^{\infty} b_{j} \partial^{-j} \mathcal{D}+\sum_{j=1}^{\infty} f_{j} \partial^{-j}
$$

expressed in terms of a bosonic "dressing" operator $\mathcal{W}$ :

$$
\mathcal{L}=\mathcal{W D}^{-1} \quad, \quad \mathcal{W}=1+\sum_{j=1}^{\infty} w_{\frac{j}{2}} \mathcal{D}^{-j} \equiv 1+\sum_{j=1}^{\infty} \alpha_{j} \partial^{-j} \mathcal{D}+\sum_{j=1}^{\infty} \beta_{j} \partial^{-j}
$$

where $b_{j}, \beta_{j}$ are bosonic superfield functions whereas $f_{j}, \alpha_{j}$ are fermionic ones and where:

$f_{0}=2 \alpha_{1} \quad, \quad b_{1}=-\mathcal{D}_{\theta} \alpha_{1} \quad, \quad f_{1}=2 \alpha_{2}-\alpha_{1} \mathcal{D}_{\theta} \alpha_{1}-2 \alpha_{1} \beta_{1}-\mathcal{D}_{\theta} \beta_{1} \quad, \quad b_{2}=\mathcal{D}_{\theta}\left(-\alpha_{2}+\alpha_{1} \beta_{1}\right)+\left(\mathcal{D}_{\theta} \alpha_{1}\right)^{2}$

and so on. The square of $\mathcal{L}$ (四) is a bosonic super-pseudo-differential operator of the form:

$$
\begin{gathered}
\mathcal{L}^{2}=\mathcal{W} \partial \mathcal{W}^{-1}=\partial+\sum_{j=1}^{\infty} u_{\frac{j}{2}} \mathcal{D}^{-j} \\
u_{\frac{1}{2}}=-\partial_{x} \alpha_{1}=\mathcal{D}_{\theta} b_{1} \quad, \quad u_{1}=-\partial_{x} \beta_{1}+\alpha_{1} \partial_{x} \alpha_{1}=2 b_{2}-b_{1}^{2}+\mathcal{D}_{\theta} f_{1}
\end{gathered}
$$

and so on.

The Lax evolution equations for MR-SKP read [4:

$$
\begin{aligned}
\frac{\partial}{\partial t_{l}} \mathcal{L} & =-\left[\mathcal{L}_{-}^{2 l}, \mathcal{L}\right]=\left[\mathcal{L}_{+}^{2 l}, \mathcal{L}\right] \\
D_{n} \mathcal{L} & =-\left\{\mathcal{L}_{-}^{2 n-1}, \mathcal{L}\right\}=\left\{\mathcal{L}_{+}^{2 n-1}, \mathcal{L}\right\}-2 \mathcal{L}^{2 n} \\
\frac{\partial}{\partial t_{l}} \mathcal{W} & =-\left(\mathcal{W} \partial^{l} \mathcal{W}^{-1}\right)_{-} \mathcal{W}, \quad D_{n} \mathcal{W}=-\left(\mathcal{W D}^{2 n-1} \mathcal{W}^{-1}\right)_{-} \mathcal{W}
\end{aligned}
$$

with the short-hand notations for the fermionic isospectral flows $D_{n}\left(\frac{\partial}{\partial t_{k}}\right.$ being the bosonic isospectral flows) :

$$
\begin{array}{r}
D_{n}=\frac{\partial}{\partial \theta_{n}}-\sum_{k=1}^{\infty} \theta_{k} \frac{\partial}{\partial t_{n+k-1}} \quad, \quad\left\{D_{k}, D_{l}\right\}=-2 \frac{\partial}{\partial t_{k+l-1}} \\
(t, \theta) \equiv\left(t_{1} \equiv x, t_{2}, \ldots ; \theta, \theta_{1}, \theta_{2}, \ldots\right)
\end{array}
$$

The super-Baker-Akhiezer (super-BA) and the adjoint super-BA wave functions are defined as:

$$
\psi_{B A}(t, \theta ; \lambda, \eta)=\mathcal{W}\left(\psi_{B A}^{(0)}(t, \theta ; \lambda, \eta)\right) \quad, \quad \psi_{B A}^{*}(t, \theta ; \lambda, \eta)=\mathcal{W}^{*-1}\left(\psi_{B A}^{*(0)}(t, \theta ; \lambda, \eta)\right)
$$


(with $\eta$ being a fermionic "spectral" parameter), in terms of the "free" super-BA functions:

$$
\begin{array}{r}
\psi_{B A}^{(0)}(t, \theta ; \lambda, \eta) \equiv e^{\xi(t, \theta ; \lambda, \eta)}, \quad \psi_{B A}^{*(0)}(t, \theta ; \lambda, \eta) \equiv e^{-\xi(t, \theta ; \lambda, \eta)} \\
\xi(t, \theta ; \lambda, \eta)=\sum_{l=1}^{\infty} \lambda^{l} t_{l}+\eta \theta+(\eta-\lambda \theta) \sum_{n=1}^{\infty} \lambda^{n-1} \theta_{n}
\end{array}
$$

For the latter it holds:

$$
\frac{\partial}{\partial t_{k}} \psi_{B A}^{(0)}=\partial_{x}^{k} \psi_{B A}^{(0)} \quad, \quad D_{n} \psi_{B A}^{(0)}=\mathcal{D}_{\theta}^{2 n-1} \psi_{B A}^{(0)}=\partial_{x}^{n-1} \mathcal{D}_{\theta} \psi_{B A}^{(0)}
$$

Because of (17) (adjoint) super-BA wave functions satisfy:

$$
\left(\mathcal{L}^{2}\right)^{(*)} \psi_{B A}^{(*)}=\lambda \psi_{B A}^{(*)} \quad, \quad \frac{\partial}{\partial t_{l}} \psi_{B A}^{(*)}= \pm\left(\mathcal{L}^{2 l}\right)_{+}^{(*)}\left(\psi_{B A}^{(*)}\right) \quad, \quad D_{n} \psi_{B A}^{(*)}= \pm\left(\mathcal{L}^{2 n-1}\right)_{+}^{(*)}\left(\psi_{B A}^{(*)}\right)
$$

Unlike the purely bosonic case [40], the superspace tau-function $\tau(t, \theta)$ is related to the superBA function in a more complicated manner [7]. On the other hand, $\tau(t, \theta)$ is simply expressed through super-residua of the pertinent super-Lax operator (44) as follows :

$$
\mathcal{R} e s \mathcal{L}^{2 k}=\frac{\partial}{\partial t_{k}} \mathcal{D}_{\theta} \ln \tau \quad, \quad \mathcal{R} e s \mathcal{L}^{2 k-1}=D_{k} \mathcal{D}_{\theta} \ln \tau
$$

A basic object in our construction is the notion of (adjoint) super-eigenfunctions $\Phi(t, \theta)$ and $\Psi(t, \theta)$, whose defining equations read:

$$
\frac{\partial}{\partial t_{l}} \Phi=\mathcal{L}_{+}^{2 l}(\Phi) \quad, \quad D_{n} \Phi=\mathcal{L}_{+}^{2 n-1}(\Phi) \quad ; \quad \frac{\partial}{\partial t_{l}} \Psi=-\left(\mathcal{L}^{2 l}\right)_{+}^{*}(\Psi) \quad, \quad D_{n} \Psi=-\left(\mathcal{L}^{2 n-1}\right)_{+}^{*}(\Psi)
$$

Following the line of argument in ref. 39] for the purely bosonic case, one can prove that any (adjoint) super-eigenfunction possesses a supersymmetric "spectral" representations:

$$
\Phi(t, \theta)=\int d \lambda d \eta \varphi(\lambda, \eta) \psi_{B A}(t, \theta ; \lambda, \eta) \quad, \quad \Psi(t, \theta)=\int d \lambda d \eta \varphi^{*}(\lambda, \eta) \psi_{B A}^{*}(t, \theta ; \lambda, \eta)
$$

with appropriate superspace "spectral" densities $\varphi(\lambda, \eta)$ and $\varphi^{*}(\lambda, \eta)$. In particular, from (18) we note that the (adjoint) super-BA functions are special examples of (adjoint) super-eigenfunctions which in addition to (20) satisfy spectral equations - the first Eqs.(18).

In what follows we shall encounter another basic object of the form $\mathcal{D}_{\theta}^{-1}(\Phi \Psi)=\mathcal{D}_{\theta} \partial_{x}^{-1}(\Phi \Psi)$ where $\Phi, \Psi$ is a pair of super-eigenfunction and adjoint super-eigenfunction. Similarly to the purely bosonic case [32] one can show that application of inverse derivative on such products is welldefined. Namely, there exists a unique superfield function - supersymmetric "squared eigenfunction potential" (super-SEP) $S(\Phi, \Psi)$ such that: $\mathcal{D}_{\theta} S(\Phi, \Psi)=\Phi \Psi$. More precisely the super-SEP satisfies the relations:

$$
\frac{\partial}{\partial t_{k}} S(\Phi, \Psi)=\mathcal{R} e s\left(\mathcal{D}^{-1} \Psi \mathcal{L}^{2 k} \Phi \mathcal{D}^{-1}\right) \quad, \quad D_{n} S(\Phi, \Psi)=\mathcal{R} e s\left(\mathcal{D}^{-1} \Psi \mathcal{L}^{2 n-1} \Phi \mathcal{D}^{-1}\right)
$$

In particular, eqs.(22) for $k=1$ and $n=1$ read:

$$
\partial_{x} S(\Phi, \Psi)=\mathcal{R} e s\left(\mathcal{D}^{-1} \Psi \mathcal{L}^{2} \Phi \mathcal{D}^{-1}\right)=\mathcal{D}_{\theta}(\Phi \Psi) \quad, \quad D_{1} S(\Phi, \Psi)=\mathcal{R} e s\left(\mathcal{D}^{-1} \Psi \mathcal{L} \Phi \mathcal{D}^{-1}\right)=\Phi \Psi
$$


It is in this well-defined sense that we will be using in the sequel inverse superspace derivatives $\mathcal{D}_{\theta}^{-1}=\mathcal{D}_{\theta} \partial^{-1}$ acting on products of super-eigenfunctions with adjoint super-eigenfunctions.

\section{Constrained Supersymmetric KP Hierarchies. Inverse Powers of Super-Lax Oper- ators}

\section{1 $\operatorname{SKP}_{\left(R ; M_{B}, M_{F}\right)}$ Hierarchies}

In ref. 14] we introduced a class of reductions of the original MR-SKP hierarchy, called $S K P_{\left(R ; M_{B}, M_{F}\right)}$ constrained super-KP models, which contain the supersymmetric extensions of various basic bosonic integrable hierarchies such as (modified) Korteveg-de-Vries, nonlinear Schrödinger (AKNS hierarchy in general), Yajima-Oikawa, coupled Boussinesq-type equations etc.. The $\operatorname{SKP}_{\left(R ; M_{B}, M_{F}\right)}$ hierarchies are defined by the following superspace Lax operators (we will use slighty different notations from [14) :

$$
\mathcal{L} \equiv \mathcal{L}_{\left(R ; M_{B}, M_{F}\right)}=\mathcal{D}^{R}+\sum_{j=0}^{R-1} v_{\frac{j}{2}} \mathcal{D}^{j}+\sum_{i=1}^{M} \Phi_{i} \mathcal{D}^{-1} \Psi_{i} \quad, \quad M \equiv M_{B}+M_{F}
$$

where $M_{B, F}$ indicate the number of bosonic/fermionic super-eigenfunctions $\Phi_{i}$ entering the purely pseudo-differential part of $\mathcal{L}_{\left(R ; M_{B}, M_{F}\right)}$. $S K P_{\left(R ; M_{B}, M_{F}\right)}$ hierarchies defined by fermionic/bosonic super-Lax operators (24), for which $R \equiv 2 r+1, M \equiv M_{B}+M_{F} \equiv 2 N+1$ and $R \equiv 2 r, M \equiv$ $M_{B}+M_{F} \equiv 2 N$, respectively, will be called in what follows "fermionic" / "bosonic" hierarchies for brevity.

One of the main results in [14] was to show that the original fermionic flows $D_{n}$ (10) for the general unconstrained MR-SKP hierarchy do not anymore define consistent flows on the space of fermionic constrained $S K P_{\left(R ; M_{B}, M_{F}\right)}$ hierarchies, i.e., the odd flows (10) do not preserve the constrained form of fermionic super-Lax operatores $\mathcal{L} \equiv \mathcal{L}_{\left(R ; M_{B}, M_{F}\right)}(24)$. We found the following consistent modification for $D_{n}$ :

$$
D_{n} \mathcal{L}=-\left\{\mathcal{L}_{-}^{2 n-1}-X_{2 n-1}, \mathcal{L}\right\}=\left\{\mathcal{L}_{+}^{2 n-1}+X_{2 n-1}, \mathcal{L}\right\}-2 \mathcal{L}^{2 n}
$$

where:

$$
\begin{gathered}
X_{2 n-1} \equiv 2 \sum_{i=1}^{M}(-1)^{|i|} \sum_{s=0}^{n-2} \mathcal{L}^{2(n-s)-3}\left(\Phi_{i}\right) \mathcal{D}^{-1}\left(\mathcal{L}^{2 s+1}\right)^{*}\left(\Psi_{i}\right) \\
D_{n} \Phi_{i}=\mathcal{L}_{+}^{2 n-1}\left(\Phi_{i}\right)-2 \mathcal{L}^{2 n-1}\left(\Phi_{i}\right)+X_{2 n-1}\left(\Phi_{i}\right) \\
D_{n} \Psi_{i}=-\left(\mathcal{L}^{2 n-1}\right)_{+}^{*}\left(\Psi_{i}\right)+2\left(\mathcal{L}^{2 n-1}\right)^{*}\left(\Psi_{i}\right)-\left(X_{2 n-1}\right)^{*}\left(\Psi_{i}\right)
\end{gathered}
$$

with the subscript $|i|$ in Eq.(26) and below denoting the Grassmann parity of the corresponding (adjoint) super-eigenfunction $\Phi_{i}, \Psi_{i}$. The modified fermionic isospectral flows $D_{n}$ (25)-(26) obey the anti-commutation algebra:

$$
\left\{D_{n}, D_{m}\right\}=-2 \partial / \partial t_{R(n+m-1)}
$$

where $\frac{\partial}{\partial t_{l}}$ are the bosonic isospectral flows for $\mathcal{L} \equiv \mathcal{L}_{\left(R ; M_{B}, M_{F}\right)}$ :

$$
\frac{\partial}{\partial t_{l}} \mathcal{L}=\left[\left(\mathcal{L}^{\frac{2 l}{R}}\right)_{+}, \mathcal{L}\right]
$$


In checking the consistency of the new $D_{n}$-flows an extensive use is made of the following superspace pseudo-differential operator identities:

$$
\mathcal{Z}_{(i, j)} \mathcal{Z}_{(k, l)}=\mathcal{Z}_{(i, j)}\left(\Phi_{k}\right) \mathcal{D}^{-1} \Psi_{l}+(-1)^{|j|(|k|+|l|+1)} \Phi_{i} \mathcal{D}^{-1} \mathcal{Z}_{(k, l)}^{*}\left(\Psi_{j}\right)
$$

where $\mathcal{Z}_{(k, l)} \equiv \Phi_{k} \mathcal{D}^{-1} \Psi_{l}$. They will turn very important in our symmetry-flows construction below. In particular, using identities (31) we get the relations:

$$
\left(\mathcal{L}^{K}\right)_{-} \equiv\left(\mathcal{L}_{\left(R ; M_{B}, M_{F}\right)}^{K}\right)_{-}=\sum_{i=1}^{M} \sum_{s=0}^{K-1}(-1)^{s|i|} \mathcal{L}^{K-1-s}\left(\Phi_{i}\right) \mathcal{D}^{-1}\left(\mathcal{L}^{s}\right)^{*}\left(\Psi_{i}\right)
$$

for the purely pseudo-differential part of arbitrary positive integer power of a fermionic super-Lax operator (24).

In the case of bosonic constrained $\operatorname{SKP}_{\left(R ; M_{B}, M_{F}\right)}$ (i.e., $R=2 r$ and $M \equiv M_{B}+M_{F}=2 N$ in (24)), we can split the set of super-eigenfunctions entering the negative pseudo-differential part of (24) in bosonic $\left\{\Phi_{a}, \Psi_{b}\right\}_{a, b=1}^{M_{B, F}}$ and femionic $\left\{\widetilde{\Phi}_{b}, \widetilde{\Psi}_{a}\right\}_{a, b=1}^{M_{B, F}}$ subsets, respectively, so that the expression (24) acquires the form:

$$
\left.L \equiv \mathcal{L}_{\left(2 r ; M_{B}, M_{F}\right)}\right|_{M_{B}+M_{F}=2 N}=L_{+}+\sum_{a=1}^{M_{B}} \Phi_{a} \mathcal{D}^{-1} \widetilde{\Psi}_{a}+\sum_{b=1}^{M_{F}} \widetilde{\Phi}_{b} \mathcal{D}^{-1} \Psi_{b}
$$

Henceforth we will use the short-hand notation $L$ to indicate bosonic super-Lax operators (33). Before proceeding let us recall that in the case of bosonic $\operatorname{SKP}_{\left(R ; M_{B}, M_{F}\right)}$ hierarchies there is no need of modification (25)-(26) of the original MR-SKP fermionic isospectral flows $D_{n}$ (10) since they preserve the constrained form of the bosonic super-Lax operator (33) unlike the case with fermionic $\operatorname{SKP}_{\left(R ; M_{B}, M_{F}\right)}$ hierarchies:

$$
\begin{gathered}
\frac{\partial}{\partial t_{l}} L=\left[(L)_{+}^{\frac{l}{r}}, L\right] \quad, \quad D_{n} \mathcal{L}_{B}=\left[(L)_{+}^{\frac{2 n-1}{2 r}}, L\right] \\
\left.D_{n} \stackrel{(\sim)}{\Phi_{a}}=(L)_{+}^{\frac{2 n-1}{2 r}} \stackrel{(\sim)}{\left(\Phi_{a}\right.}\right) \quad, \quad D_{n} \stackrel{(\sim)}{\Psi}_{a}=-\left(L^{\frac{2 n-1}{2 r}}\right)_{+}^{*}\left(\stackrel{(\sim)}{\Psi_{a}}\right)
\end{gathered}
$$

Note that the $2 r$-th root $L^{\frac{1}{2 r}}$ of the bosonic super-Lax operator (33) (and similarly for the higher $\frac{2 n-1}{2 r}$ powers thereof) is a fermionic super-pseudo-differential operator of the general Manin-Radul form (雨) whose coefficients are determined recursively from the relation $\left(L^{\frac{1}{2 r}}\right)^{2 r}=L$.

We will also need the explicit expressions for inverse powers of $\operatorname{SKP}_{\left(R ; M_{B}, M_{F}\right)}$ super-Lax operators (24). Following the same lines of the construction in ref. [42] of inverse powers of KP Lax operators in the purely bosonic case, we can represent the super-Lax operator (24) as a ratio of two purely super-differential operators $L_{\frac{1}{2}(R+M)}$ and $L_{\frac{1}{2} M}$ of orders $\frac{1}{2}(R+M)$ and $\frac{1}{2} M$, respectively:

$$
\mathcal{L}_{\left(R ; M_{B}, M_{F}\right)}=L_{\frac{1}{2}(R+M)} L_{\frac{1}{2} M}^{-1}=\left\{\begin{array}{lr}
L_{N+r+1} L_{N+\frac{1}{2}}^{-1} & M \equiv M_{B}+M_{F}=2 N+1, R=2 r+1 \\
L_{N+r} L_{N}^{-1} & M \equiv M_{B}+M_{F}=2 N, R=2 r
\end{array}\right.
$$

where the first line refers to fermionic super-Lax operator and the second line refers to bosonic superLax operator, respectively. According to [5] (see also refs. 110, 15]), any super-differential operators $L_{N}$ (bosonic, of integer order) and $L_{N+\frac{1}{2}}$ (fermionic, of half-integer order) can be parametrized through the elements of their respective kernels:

$$
\operatorname{Ker}\left(L_{N}\right)=\left\{\varphi_{0}, \varphi_{\frac{1}{2}}, \ldots, \varphi_{N-1}, \varphi_{N-\frac{1}{2}}\right\} \quad, \quad \operatorname{Ker}\left(L_{N+\frac{1}{2}}\right)=\left\{\varphi_{0}, \varphi_{\frac{1}{2}}, \ldots, \varphi_{N-1}, \varphi_{N-\frac{1}{2}}, \varphi_{N}\right\}
$$


as follows:

$$
L_{N}=\mathcal{T}_{\varphi_{N-\frac{1}{2}}}^{(2 N-1)} \mathcal{T}_{\varphi_{N-1}}^{(2 N-2)} \ldots \mathcal{T}_{\varphi_{\frac{1}{2}}}^{(1)} \mathcal{T}_{\varphi_{0}}^{(0)} \quad, \quad L_{N+\frac{1}{2}}=\mathcal{T}_{\varphi_{N}}^{(2 N)} \mathcal{T}_{\varphi_{N-\frac{1}{2}}}^{(2 N-1)} \ldots \mathcal{T}_{\varphi_{\frac{1}{2}}}^{(1)} \mathcal{T}_{\varphi_{0}}^{(0)}
$$

with the notations $(j=0,1, \ldots, 2 N)$ :

$$
\mathcal{T}_{\varphi_{\frac{j}{2}}}^{(j)} \equiv \varphi_{\frac{j}{2}}^{(j)} \mathcal{D}\left(\varphi_{\frac{j}{2}}^{(j)}\right)^{-1} \quad, \quad \varphi_{\frac{j}{2}}^{(j)} \equiv \mathcal{T}_{\frac{(j-1)}{2}}^{(j-1)} \mathcal{T}_{\varphi_{\frac{j}{2}-1}^{(j-2)}}^{\left(j-\mathcal{T}_{\varphi_{\frac{1}{2}}}^{(1)}\right.} \mathcal{T}_{\varphi_{0}}^{(0)}\left(\varphi_{\frac{j}{2}}\right)
$$

Integer/half-integer indices of the corresponding elements of the kernels indicate that the latter are bosonic/fermionic, respectively. On the other hand all super-functions $\varphi_{\frac{j}{2}}^{(j)}$ in (38) are bosonic for any index $j$. As shown in [10, 15], the objects $\varphi_{\frac{j}{2}}^{(j)}$ have explicit representations as ratios of Wronskian-like Berezinians (super-determinants) (see also Section 10 below).

Furthermore, as in the purely bosonic case 442] one can show that the inverse power of $L_{N}$ is given as:

$$
L_{N}^{-1}=\sum_{\alpha=1}^{N}\left[\varphi_{\alpha} \mathcal{D}^{-1} \widetilde{\psi}_{\alpha}+\widetilde{\varphi}_{\alpha} \mathcal{D}^{-1} \psi_{\alpha}\right]
$$

where the set of super-functions $\left\{\varphi_{\alpha}, \widetilde{\varphi}_{\alpha}\right\}_{\alpha=1}^{N}$ is spanning $\operatorname{Ker}\left(L_{N}\right)$, whereas the super-functions $\left\{\psi_{\alpha}, \widetilde{\psi}_{\alpha}\right\}_{\alpha=1}^{N}$ span $\operatorname{Ker}\left(L_{N}^{*}\right)$ - the kernel of the adjoint operator, and where we have split explicitly the corresponding kernel elements into bosonic and fermionic (indicated by "tilde") subsets.

\subsection{Inverse Powers of Bosonic Super-Lax Operators}

We are now ready to write the explicit expressions for inverse powers of the super-Lax operators (24) (cf. Eq.(36)). We start with the bosonic super-Lax operators $\mathcal{L}_{\left(R ; M_{B}, M_{F}\right)}$ (33) where $R=2 r$, $M \equiv M_{B}+M_{F}=2 N$. Henceforth we will use the short-hand notation $L \equiv \mathcal{L}_{\left(R ; M_{B}, M_{F}\right)}$ for the latter. Taking into account (39) and the identities (31) we obtain:

$$
L^{-1}=L_{N} L_{N+r}^{-1}=\sum_{\beta=1}^{N+r}\left[L_{N}\left(\varphi_{\beta}\right) \mathcal{D}^{-1} \widetilde{\psi}_{\beta}+L_{N}\left(\widetilde{\varphi}_{\beta}\right) \mathcal{D}^{-1} \psi_{\beta}\right]
$$

where the sets of super-functions $\left\{\varphi_{\beta}, \widetilde{\varphi}_{\beta}\right\}_{\beta=1}^{N+r}$ and $\left\{\psi_{\beta}, \widetilde{\psi}_{\beta}\right\}_{\beta=1}^{N+r}$ span the kernels $\operatorname{Ker}\left(L_{N+r}\right)$ and $\operatorname{Ker}\left(L_{N+r}^{*}\right)$, respectively. For later convenience it is useful to introduce the following short hand notations:

$$
\begin{array}{lll}
\Phi_{\beta}^{(-m)} \equiv L^{-(m-1)}\left(L_{N}\left(\varphi_{\beta}\right)\right) & , & \Psi_{\beta}^{(-m)} \equiv\left(L^{-(m-1)}\right)^{*}\left(\psi_{\beta}\right) \\
\widetilde{\Phi}_{\beta}^{(-m)} \equiv L^{-(m-1)}\left(L_{N}\left(\widetilde{\varphi}_{\beta}\right)\right) & , & \widetilde{\Psi}_{\beta}^{(-m)} \equiv\left(L^{-(m-1)}\right)^{*}\left(\widetilde{\psi}_{\beta}\right)
\end{array}
$$

where $m=1,2, \ldots$, and $\beta=1,2, \ldots, N+r$. Note that the superfunctions in (41) and (42) are bosonic and fermionnic, respectively. In terms of the short-hand notations (41)-(42), we can write the explicit expression for arbitrary integer $K \geq 1$ inverse power of $L$ generalizing (40) as:

$$
L^{-K}=\sum_{\beta=1}^{N+r} \sum_{s=1}^{K}\left[\Phi_{\beta}^{(-K-1+s)} \mathcal{D}^{-1} \widetilde{\Psi}_{\beta}^{(-s)}+\widetilde{\Phi}_{\beta}^{(-K-1+s)} \mathcal{D}^{-1} \Psi_{\beta}^{(-s)}\right]
$$


The latter equality is completely analogous to the expression for the purely pseudo-differential part of arbitrary positive integer powers of $L$ (cf. Eq.(32)):

$$
L^{K}=\sum_{s=1}^{K}\left[\sum_{a=1}^{M_{B}} \Phi_{a}^{(K+1-s)} \mathcal{D}^{-1} \widetilde{\Psi}_{a}^{(s)}+\sum_{b=1}^{M_{F}} \widetilde{\Phi}_{b}^{(K+1-s)} \mathcal{D}^{-1} \Psi_{b}^{(s)}\right]
$$

where we introduced another set of convenient short-hand notations similar to (41)-(42) :

$$
\Phi_{a}^{(m)} \equiv L^{m-1}\left(\Phi_{a}\right) \quad, \quad \Psi_{b}^{(m)} \equiv\left(L^{m-1}\right)^{*}\left(\Psi_{b}\right) \quad, \quad \widetilde{\Phi}_{b}^{(m)} \equiv L^{m-1}\left(\widetilde{\Phi}_{b}\right) \quad, \quad \widetilde{\Psi}_{a}^{(m)} \equiv\left(L^{m-1}\right)^{*}\left(\widetilde{\Psi}_{a}\right)
$$

with $m=1,2, \ldots, a=1, \ldots, M_{B}$ and $b=1, \ldots, M_{F}$. The derivation of both Eqs.(43)-(44) is based on systematic use of identities (31).

In what follows an essential use will be made of the following simple consequences from the definitions of the corresponding objects above:

$$
L\left(L_{N}\left(\stackrel{(\sim)}{\varphi}_{\beta}\right)\right)=0 \quad, \quad L^{*}\left(\stackrel{(\sim)}{\psi}_{\beta}\right)=0 \quad, \quad L^{-1}\left(\stackrel{(\sim)}{\Phi_{a}}\right)=0 \quad, \quad\left(L^{-1}\right)^{*}\left(\stackrel{(\sim)}{\Psi_{a}}\right)=0
$$

or, more generally, for the objects defined in (41)-(42) and (45) :

$$
\begin{aligned}
& L^{K}\left(\stackrel{(\sim)}{\Phi}_{\beta}^{(-m)}\right)=0 \quad, \quad\left(L^{*}\right)^{K}\left(\stackrel{(\sim)}{\Psi}_{\beta}^{(-m)}\right)=0 \quad, \\
& L^{-K}\left(\stackrel{(\sim)}{\Phi_{a}^{(m)}}\right)=0 \quad, \quad\left(L^{*}\right)^{-K}\left(\stackrel{(\sim)}{\Psi}_{a}^{(m)}\right)=0 \quad \text { for any } K \geq m
\end{aligned}
$$

For later use we also observe, that the supersymmetric isospectral flow equations written for the inverse $L^{-1}$ of the bosonic super-Lax operator (40) :

$$
\frac{\partial}{\partial t_{l}} L^{-1}=\left[\left(L^{\frac{l}{r}}\right)_{+}, L^{-1}\right] \quad, \quad D_{n} L^{-1}=\left[\left(L^{\frac{2 n-1}{2 r}}\right)_{+}, L^{-1}\right]
$$

straightforwardly imply (upon using identities (31)) that the super-functions $\left\{\stackrel{(\sim)}{\Phi}_{b}^{(-m)}, \stackrel{(\sim)}{\Psi}_{b}^{(-m)}\right\}$ (41) - (42) entering the various inverse powers (43) of $L$ (33) are (adjoint) super-eigenfunctions (20) of the latter:

$$
\begin{aligned}
& \frac{\partial}{\partial t_{l}} \stackrel{(\sim)}{\Phi}_{b}^{(-m)}=\left(L^{\frac{l}{r}}\right)_{+}\left(\stackrel{(\sim)}{\Phi}_{b}^{(-m)}\right) \quad, \quad D_{n} \stackrel{(\sim)}{\Phi}_{b}^{(-m)}=\left(L^{\frac{2 n-1}{2 r}}\right)_{+}\left(\stackrel{\left(\sim^{(}\right)}{(-m)}\right) \\
& \frac{\partial}{\partial t_{l}} \stackrel{(\sim)}{\Psi}_{b}^{(-m)}=-\left(L^{\frac{l}{r}}\right)_{+}^{*}\left(\stackrel{(\sim)}{\Phi_{b}^{(-m)}}\right) \quad, \quad D_{n} \stackrel{(\sim)}{\Phi}_{b}^{(-m)}=-\left(L^{\frac{2 n-1}{2 r}}\right)_{+}^{*}\left(\stackrel{(\sim)}{\Phi_{b}^{(-m)}}\right)
\end{aligned}
$$

(cf. Eqs.(57) below for the analogous result in the case of fermionic $\operatorname{SKP}_{\left(R ; M_{B}, M_{F}\right)}$ hierarchies).

\subsection{Inverse Powers of Fermionic Super-Lax Operators}

Repeating the same steps as in the derivation of Eqs.(40) and (43), we obtain the explicit expressions for the inverse powers of fermionic super-Lax operators (24):

$$
\begin{gathered}
\mathcal{L}^{-1}=L_{N+\frac{1}{2}} L_{N+r+1}^{-1}= \\
=\sum_{\alpha=1}^{N+r+1}\left[L_{N+\frac{1}{2}}\left(\varphi_{\alpha}\right) \mathcal{D}^{-1} \widetilde{\psi}_{\alpha}+L_{N+\frac{1}{2}}\left(\widetilde{\varphi}_{\alpha}\right) \mathcal{D}^{-1} \psi_{\alpha}\right] \equiv \sum_{I=1}^{2(N+r+1)} \phi_{I} \mathcal{D}^{-1} \psi_{I}
\end{gathered}
$$




$$
\mathcal{L}^{-K}=\sum_{I=1}^{2(N+r+1)} \sum_{s=0}^{K-1}(-1)^{s|I|} \phi_{I}^{(-(K-1-s) / 2)} \mathcal{D}^{-1} \psi_{I}^{(-s / 2)}
$$

where:

$$
\left\{\varphi_{\alpha}, \widetilde{\varphi}_{\alpha}\right\}_{\alpha=1}^{N+r+1} \equiv\left\{\varphi_{I}\right\}_{I=1}^{2(N+r+1)} \quad, \quad\left\{\psi_{\alpha}, \widetilde{\psi}_{\alpha}\right\}_{\alpha=1}^{N+r+1} \equiv\left\{\psi_{I}\right\}_{I=1}^{2(N+r+1)}
$$

span the kernels $\operatorname{Ker}\left(L_{N+r+1}\right)$ and $\operatorname{Ker}\left(L_{N+r+1}^{*}\right)$, respectively, and where we have introduced further short-hand notations analogous to (41)-(42) and (45) :

$$
\begin{gathered}
\phi_{I} \equiv L_{N+\frac{1}{2}}\left(\varphi_{I}\right) \quad, \quad \phi_{I}^{(-\ell / 2)} \equiv \mathcal{L}^{-\ell}\left(\phi_{I}\right) \quad, \quad \psi_{I}^{(-\ell / 2)} \equiv\left(\mathcal{L}^{-\ell}\right)^{*}\left(\psi_{I}\right) \\
\Phi_{i}^{(\ell / 2)} \equiv \mathcal{L}^{\ell}\left(\Phi_{i}\right) \quad, \quad \Psi_{i}^{(\ell / 2)} \equiv\left(\mathcal{L}^{\ell}\right)^{*}\left(\Psi_{i}\right)
\end{gathered}
$$

Furthermore, similar to (46)-(47) the following relations hold for fermionic $\operatorname{SKP}_{\left(R ; M_{B}, M_{F}\right)}$ hierarchies:

$$
\mathcal{L}\left(\phi_{I}\right)=0 \quad, \quad \mathcal{L}^{*}\left(\psi_{I}\right)=0 \quad, \quad \mathcal{L}^{-1}\left(\Phi_{i}\right)=0 \quad, \quad\left(\mathcal{L}^{-1}\right)^{*}\left(\Psi_{i}\right)=0
$$

Acting with the isospectral flows $\frac{\partial}{\partial t_{l}}$ and $D_{n}$ on the inverse powers of the fermionic super-Lax operator (50) (cf. (25) and (30)) :

$$
\frac{\partial}{\partial t_{l}} \mathcal{L}^{-1}=\left[\mathcal{L}_{+}^{\frac{2 l}{R}}, \mathcal{L}^{-1}\right] \quad, \quad D_{n} \mathcal{L}^{-1}=\left\{-\mathcal{L}_{-}^{2 n-1}+X_{2 n-1}, \mathcal{L}^{-1}\right\}
$$

and taking into account identities (31) together with (55), we deduce that the sets of super-functions $\left\{\phi_{I}\right\} \equiv\left\{L_{n+\frac{1}{2}}\left(\stackrel{(\sim)}{\varphi}_{\alpha}\right)\right\}$ and $\left\{\psi_{I}\right\} \equiv\{\stackrel{(\sim)}{\psi} \alpha\}$ are (adjoint) super-eigenfunctions of the fermionic $\operatorname{SKP}_{\left(R ; M_{B}, M_{F}\right)}$ hierarchy (cf. Eqs.(27)-(28)) :

$$
\begin{array}{r}
\frac{\partial}{\partial t_{l}} \phi_{I}=\mathcal{L}_{+}^{\frac{2 l}{R}}\left(\phi_{I}\right) \quad, \quad D_{n} \phi_{I}=\left[\mathcal{L}_{+}^{2 n-1}+X_{2 n-1}\right]\left(\phi_{I}\right) \\
\frac{\partial}{\partial t_{l}} \psi_{I}=-\left(\mathcal{L}^{\frac{2 l}{R}}\right)_{+}^{*}\left(\psi_{I}\right) \quad, \quad D_{n} \psi_{I}=-\left[\left(\mathcal{L}^{2 n-1}\right)_{+}^{*}+X_{2 n-1}^{*}\right]\left(\psi_{I}\right)
\end{array}
$$

Analogous result holds also for the super-functions $\stackrel{(\sim)}{\Phi}_{b}^{(-m)}$ (41) and $\stackrel{(\sim)}{\Psi}_{b}^{(-m)}$ (42), connected with the bosonic $\operatorname{SKP}_{\left(R ; M_{B}, M_{F}\right)}$ hierarchies (33) (see Eqs.(49) in Section 6 below).

\section{Additional Symmetries for Super-KP Hierarchies: General Formalism}

Bosonic/fermionic flows $\delta_{B, F}$ on the space of Sato super-pseudo-differential Lax operators $\mathcal{L}$ (41) or, equivalently, on the space of Sato super-dressing operators $\mathcal{W}$ (5) are defined (similarly to the purely bosonic case, see e.g. first ref. [22]) in terms of bosonic/fermionic super-pseudo-differential operators $\mathcal{M}_{B, F}$ by:

$$
\delta_{B} \mathcal{L}=\left[\mathcal{M}_{B}, \mathcal{L}\right] \quad, \quad \delta_{F} \mathcal{L}=\left\{\mathcal{M}_{F}, \mathcal{L}\right\} \quad ; \quad \delta_{B, F} \mathcal{W}=\mathcal{M}_{B, F} \mathcal{W}
$$

where $\mathcal{M}_{B, F}$ are bosonic/fermionic purely super-pseudo-differential operators. A flow $\delta_{B, F}$ (58) is a symmetry of MR-SKP hierarchy if and only if it (anti-)commutes with the isospectral Manin-Radul flows, which implies:

$$
\frac{\partial}{\partial t_{l}} \mathcal{M}_{B, F}=\left[\left(\mathcal{L}^{2 l}\right)_{+}, \mathcal{M}_{B, F}\right]_{-}
$$




$$
\begin{aligned}
& D_{n} \mathcal{M}_{B}=\left[\left(\mathcal{L}^{2 n-1}\right)_{+}+X_{2 n-1}, \mathcal{M}_{B}\right]_{-}+\delta_{B} X_{2 n-1} \\
& D_{n} \mathcal{M}_{F}=\left\{\left(\mathcal{L}^{2 n-1}\right)_{+}+X_{2 n-1}, \mathcal{M}_{F}\right\}_{-}-\delta_{F} X_{2 n-1}
\end{aligned}
$$

In Eqs.(61) we have taken into account the modification (25) $-(26)$ of the fermionic isospectral flows $D_{n}$ needed in the case of fermionic constrained $\operatorname{SKP}\left(R ; M_{B}, M_{F}\right)$ hierarchies (24).

Extending the construction in the first ref.[22] to the supersymmetric case, one can show that the general form of $\mathcal{M}_{B, F}$ obeying (59)-(61) can be represented in terms of as follows:

$$
\mathcal{M}_{B, F}=\int d \lambda d \eta_{1} d \mu d \eta_{2} \rho_{B, F}\left(\lambda, \eta_{1} ; \mu, \eta_{2}\right) \psi_{B A}\left(t, \theta ; \mu, \eta_{2}\right) \mathcal{D}^{-1} \psi_{B A}^{*}\left(t, \theta ; \lambda, \eta_{1}\right)=\sum_{P, Q \in \mathcal{E}} c_{P Q}^{(B, F)} \Phi_{Q} \mathcal{D}^{-1} \Psi_{P}
$$

where $\rho_{B, F}\left(\lambda, \eta_{1} ; \mu, \eta_{2}\right)$ is arbitrary (in the case of the general MR-SKP hierarchy (14)) double Laurent series in $\lambda$ and $\mu$. In the second equality above the sums run in general over an infinite set $\mathcal{E}$ of indices, and $\left\{\Phi_{P}, \Psi_{P}\right\}_{P \in \mathcal{E}}$ are (adjoint) super-eigenfunctions of the super-Lax operator $\mathcal{L}$, i.e., satisfying (20) for $\Phi=\Phi_{P}$ and $\Psi=\Psi_{P}$. The second equality in (62) arises from the general representation of the "bispectral" density:

$$
\rho_{B, F}\left(\lambda, \eta_{1} ; \mu, \eta_{2}\right)=\sum_{P, Q \in \mathcal{E}} c_{P Q}^{(B, F)} \varphi_{Q}\left(\mu, \eta_{2}\right) \psi_{P}\left(\lambda, \eta_{1}\right)
$$

with $c_{P Q}^{(B, F)}$ being constant matrices, in terms of basis of superspace functions $\left\{\varphi_{P}(\lambda, \eta)\right\}$ and $\left\{\psi_{P}(\lambda, \eta)\right\}$ (Laurent series in $\lambda$ ), taking into account the spectral representation for (adjoint) supereigenfunctions Eqs.(21).

We need furthermore to define the action of $\delta_{B, F}$-flows on (adjoint) super-eigenfunctions (20). First we note from (18) and the last Eqs.(58) that $\delta_{B, F} \psi_{B A}^{(*)}= \pm\left(\mathcal{M}_{B, F}\right)^{(*)}\left(\psi_{B A}^{(*)}\right)$. For general (adjoint) super-eigenfunctions we have:

$$
\delta_{B, F} \Phi=\mathcal{M}_{B, F}(\Phi)+\mathcal{F}^{B, F} \quad, \quad \delta_{B, F} \Psi=-\left(\mathcal{M}_{B, F}\right)^{*}(\Psi)+\mathcal{G}^{B, F}
$$

where the inhomogeneous terms $\mathcal{F}^{B, F}$ and $\mathcal{G}^{B, F}$ are other (adjoint) super-eigenfunctions (special examples are Eqs.(27)-(28)). The emergence of additional non-homogeneous terms on the r.h.s. of Eqs.(64) is due to the nontrivial (in general) action of $\delta_{B, F}$-flows on the pertinent spectral densities in (21). The form of the latter non-homogeneous terms is not arbitrary in general. Namely, when $\Phi$ and $\Psi$ are (adjoint) super-eigenfunctions entering the negative pseudo-differential parts of the super-Lax operator (24) or its inverse powers (50)-(51), then the additional terms $\mathcal{F}^{B, F}$ and $\mathcal{G}^{B, F}$ in (64) are determined uniquely from the consistency of the flow action (58) with the constrained form of $\mathcal{L}$ (24). Explicit construction of (64) with consistent non-homogeneous terms will be given in the next sections.

Finally, we find for the transformation of the super-tau-function (19) under the action of bosonic/fermionic symmetry flows :

$$
\delta_{B, F} \ln \tau=\mathcal{D}_{\theta}^{-1}\left(\mathcal{R e s} \mathcal{M}_{B, F}\right)=\sum_{P, Q \in \mathcal{E}} c_{P Q}^{(B, F)} \mathcal{D}_{\theta}^{-1}\left(\Phi_{Q} \Psi_{P}\right)
$$

\section{Superloop Superalgebra Symmetries of Constrained SKP Hierarchies: The Case of Fermionic Super-Lax Operators}


We now proceed by constructing the explicit form of additional symmetry generating super-pseudodifferential operators $\mathcal{M}_{B, F}$ (62) in the case of fermionic constrained $S K P_{\left(R ; M_{B}, M_{F}\right)}$ hierarchies (24), i.e., with super-Lax operators $\mathcal{L} \equiv \mathcal{L}_{\left(R ; M_{B}, M_{F}\right)}$ being fermionic (recall $R=2 r+1, M \equiv M_{B}+M_{F}=$ $2 N+1):$

$$
\begin{aligned}
& \mathcal{M}_{\mathcal{A}}^{(\ell / 2)} \equiv \sum_{i, j=1}^{M} \mathcal{A}_{i j}^{(\ell / 2)} \sum_{s=0}^{\ell-1}(-1)^{s(|j|+\ell)} \mathcal{L}^{\ell-1-s}\left(\Phi_{j}\right) \mathcal{D}^{-1}\left(\mathcal{L}^{s}\right)^{*}\left(\Psi_{i}\right) \\
& \mathcal{M}_{\mathcal{F}}^{(\ell / 2)} \equiv \sum_{i, j=1}^{M} \mathcal{F}_{i j}^{(\ell / 2)} \sum_{s=0}^{\ell-1}(-1)^{s(|j|+\ell)} \mathcal{L}^{\ell-1-s}\left(\Phi_{j}\right) \mathcal{D}^{-1}\left(\mathcal{L}^{s}\right)^{*}\left(\Psi_{i}\right)
\end{aligned}
$$

where $\ell=1,2, \ldots$.Here $\mathcal{A}^{(\ell / 2)}$ and $\mathcal{F}^{(\ell / 2)}$ are graded constant matrices of the following types:

(a) For $\ell=2 n$ the matrices $\mathcal{A}^{(n)}$ and $\mathcal{F}^{(n)}$ are purely bosonic and purely fermionic elements, respectively, belonging (as a vector space) to the superalgebra $G L\left(M_{B}, M_{F}\right)$ of graded $\left(M_{B}, M_{F}\right) \times$ $\left(M_{B}, M_{F}\right)$ matrices:

$$
\mathcal{A}^{(n)}=\left(\begin{array}{cc}
A^{(n)} & 0 \\
0 & D^{(n)}
\end{array}\right) \quad, \quad \mathcal{F}^{(n)}=\left(\begin{array}{cc}
0 & B^{(n)} \\
C^{(n)} & 0
\end{array}\right)
$$

Here the block matrices $A^{(n)}, B^{(n)}, C^{(n)}$ and $D^{(n)}$ are of sizes $M_{B} \times M_{B}, M_{B} \times M_{F}, M_{F} \times M_{B}$ and $M_{F} \times M_{F}$, respectively.

(b) For $\ell=2 n-1$ the matrices $\mathcal{A}^{\left(n-\frac{1}{2}\right)}$ and $\mathcal{F}^{\left(n-\frac{1}{2}\right)}$ are purely bosonic and purely fermionic elements, respectively, belonging (as a vector space) to $\widetilde{G L}\left(M_{B}, M_{F}\right)$ - the superalgebra of $\left(M_{B}, M_{F}\right) \times$ $\left(M_{B}, M_{F}\right)$ graded matrices in the "twisted" basis (the diagonal blocks are fermionic, whereas the off-diogonal blocks are bosonic; for a general discussion of non-standard formats of matrix superalgebras, see ref. 41]) :

$$
\mathcal{F}^{\left(n-\frac{1}{2}\right)}=\left(\begin{array}{cc}
B^{\left(n-\frac{1}{2}\right)} & 0 \\
0 & C^{\left(n-\frac{1}{2}\right)}
\end{array}\right) \quad, \quad \mathcal{A}^{\left(n-\frac{1}{2}\right)}=\left(\begin{array}{cc}
0 & A^{\left(n-\frac{1}{2}\right)} \\
D^{\left(n-\frac{1}{2}\right)} & 0
\end{array}\right)
$$

In this case the sizes of the block matrices $A^{\left(n-\frac{1}{2}\right)}, B^{\left(n-\frac{1}{2}\right)}, C^{\left(n-\frac{1}{2}\right)}$ and $D^{\left(n-\frac{1}{2}\right)}$ are $M_{B} \times M_{F}$, $M_{B} \times M_{B}, M_{F} \times M_{F}$ and $M_{F} \times M_{B}$, respectively.

Thus, all graded matrices (68)-(69) are special positive-grade elements of a superloop superalgebra $\widehat{G L}\left(M_{B}, M_{F}\right)$ with half-integer grading $\ell=0, \pm \frac{1}{2}, \pm 1, \pm \frac{3}{2}, \ldots$. More generally, $\widehat{G L}\left(N_{1}, N_{2}\right)$ here will denote an infinite-dimensional algebra with half-integer grading:

$$
\widehat{G L}\left(N_{1}, N_{2}\right)=\oplus_{\ell \in \mathbb{Z}^{G L^{\left(\frac{\ell}{2}\right)}}\left(N_{1}, N_{2}\right)}
$$

whose $\frac{\ell}{2}$-grade subspaces consist of super-matrices of the following form:

$$
\begin{gathered}
G L^{(n)}\left(N_{1}, N_{2}\right)=\left\{\left(\begin{array}{ll}
A^{(n)} & B^{(n)} \\
C^{(n)} & D^{(n)}
\end{array}\right) \in G L\left(N_{1}, N_{2}\right)\right\} \\
G L^{\left(n-\frac{1}{2}\right)}\left(N_{1}, N_{2}\right)=\left\{\left(\begin{array}{ll}
B^{\left(n-\frac{1}{2}\right)} & A^{\left(n-\frac{1}{2}\right)} \\
D^{\left(n-\frac{1}{2}\right)} & C^{\left(n-\frac{1}{2}\right)}
\end{array}\right) \in \widetilde{G} L\left(N_{1}, N_{2}\right)\right\}
\end{gathered}
$$

Note from Eq.(66) that for $\ell=2 n$ :

$$
\mathcal{M}_{\mathcal{A}=\mathbb{1}}^{(n)} \equiv \sum_{j=1}^{M} \sum_{s=0}^{2 n-1}(-1)^{s|j|} \mathcal{L}^{2 n-1-s}\left(\Phi_{j}\right) \mathcal{D}^{-1}\left(\mathcal{L}^{s}\right)^{*}\left(\Psi_{j}\right)=\left(\mathcal{L}^{2 n}\right)_{-}
$$


whereas for $\ell=2 n-1$ Eq.(67) implies:

$$
\mathcal{M}_{\mathcal{F}=\mathbb{1}}^{\left(n-\frac{1}{2}\right)} \equiv \sum_{j=1}^{M} \sum_{s=0}^{2 n-2}(-1)^{s(|j|+1)} \mathcal{L}^{2 n-2-s}\left(\Phi_{j}\right) \mathcal{D}^{-1}\left(\mathcal{L}^{s}\right)^{*}\left(\Psi_{j}\right)=\left(\mathcal{L}^{2 n-1}\right)_{-}-X_{2 n-1}
$$

with $X_{2 n-1}$ the same as in Eq.(26).

Now, we define the following infinite set of bosonic and fermionic flows, respectively:

$$
\delta_{\mathcal{A}}^{(\ell / 2)} \mathcal{L}=\left[\mathcal{M}_{\mathcal{A}}^{(\ell / 2)}, \mathcal{L}\right] \quad, \quad \delta_{\mathcal{F}}^{(\ell / 2)} \mathcal{L}=\left\{\mathcal{M}_{\mathcal{F}}^{(\ell / 2)}, \mathcal{L}\right\}
$$

One can show, using the superspace identities (31), that the flows (75) are well-defined, namely, that they preserve the specific constrained form of the superspace Lax operator (24) provided the action of these flows on the constituent (adjoint) super-eigenfunctions is given by:

$$
\begin{gathered}
\delta_{\mathcal{A}}^{(\ell / 2)} \Phi_{i}=\mathcal{M}_{\mathcal{A}}^{(\ell / 2)}\left(\Phi_{i}\right)-\sum_{j=1}^{M} \mathcal{A}_{i j}^{(\ell / 2)} \mathcal{L}^{\ell}\left(\Phi_{j}\right) \\
\delta_{\mathcal{A}}^{(\ell / 2)} \Psi_{i}=-\left(\mathcal{M}_{\mathcal{A}}^{(\ell / 2)}\right)^{*}\left(\Psi_{i}\right)+\sum_{j=1}^{M}(-1)^{\ell|j|} \mathcal{A}_{j i}^{(\ell / 2)}\left(\mathcal{L}^{\ell}\right)^{*}\left(\Psi_{j}\right) \\
\delta_{\mathcal{F}}^{(\ell / 2)} \Phi_{i}=\mathcal{M}_{\mathcal{F}}^{(\ell / 2)}\left(\Phi_{i}\right)+\sum_{j=1}^{M} \mathcal{F}_{i j}^{(\ell / 2)} \mathcal{L}^{\ell}\left(\Phi_{j}\right) \\
\delta_{\mathcal{F}}^{(\ell / 2)} \Psi_{i}=-\left(\mathcal{M}_{\mathcal{F}}^{(\ell / 2)}\right)^{*}\left(\Psi_{i}\right)-\sum_{j=1}^{M}(-1)^{(\ell+1)|j|} \mathcal{F}_{j i}^{(\ell / 2)}\left(\mathcal{L}^{\ell}\right)^{*}\left(\Psi_{j}\right)
\end{gathered}
$$

Furthermore, employing again identities (31), we find:

$$
\begin{gathered}
\delta_{\mathcal{A}_{1}}^{(\ell / 2)} \mathcal{M}_{\mathcal{A}_{2}}^{(m / 2)}-\delta_{\mathcal{A}_{2}}^{(m / 2)} \mathcal{M}_{\mathcal{A}_{1}}^{(\ell / 2)}-\left[\mathcal{M}_{\mathcal{A}_{1}}^{(\ell / 2)}, \mathcal{M}_{\mathcal{A}_{2}}^{(m / 2)}\right]=\mathcal{M}_{\left[\mathcal{A}_{1}, \mathcal{A}_{2}\right]}^{(\ell+m) / 2)} \\
\delta_{\mathcal{A}}^{(\ell / 2)} \mathcal{M}_{\mathcal{F}}^{(m / 2)}-\delta_{\mathcal{F}}^{(m / 2)} \mathcal{M}_{\mathcal{A}}^{(\ell / 2)}-\left[\mathcal{M}_{\mathcal{A}}^{(\ell / 2)}, \mathcal{M}_{\mathcal{F}}^{(m / 2)}\right]= \begin{cases}\mathcal{M}_{[\mathcal{A}, \mathcal{F}]}^{((\ell+m) / 2)} & \text { for } \ell=\text { even } \\
-\mathcal{M}_{\{\mathcal{A}, \mathcal{F}\}}^{(\ell+m) / 2)} & \text { for } \ell=\text { odd }\end{cases} \\
= \begin{cases}\delta_{\mathcal{F}_{1}}^{(\ell / 2)} \mathcal{M}_{\mathcal{F}_{2}}^{(m / 2)}+\delta_{\mathcal{F}_{2}}^{(m / 2)} \mathcal{M}_{\mathcal{F}_{1}}^{(\ell / 2)}-\left\{\mathcal{M}_{\mathcal{F}_{1}}^{(\ell / 2)}, \mathcal{M}_{\mathcal{F}_{2}}^{(m / 2)}\right\}= \\
\pm \mathcal{M}_{\left\{\mathcal{F}_{1}, \mathcal{F}_{2}\right\}}^{((\ell+m) / 2)} & \text { for }(\ell, m)=(\text { odd, odd }) /(\text { even, even }) \\
\left. \pm \mathcal{M}_{\left[\mathcal{F}_{1}, \mathcal{F}_{2}\right]}^{(\ell+m)}\right) & \text { for }(\ell, m)=(\text { odd, even }) /(\text { even }, \text { odd })\end{cases}
\end{gathered}
$$

which implies the following infinite-dimensional algebra of flows:

$$
\begin{gathered}
{\left[\delta_{\mathcal{A}_{1}}^{(\ell / 2)}, \delta_{\mathcal{A}_{2}}^{(m / 2)}\right]=\delta_{\left[\mathcal{A}_{1}, \mathcal{A}_{2}\right]}^{((\ell+m) / 2)}} \\
{\left[\delta_{\mathcal{A}}^{(\ell / 2)}, \delta_{\mathcal{F}}^{(m / 2)}\right]=\delta_{[\mathcal{A}, \mathcal{F}]}^{((\ell+m) / 2)} \quad \text { for } \ell=\text { even } \quad, \quad\left[\delta_{A}^{(\ell / 2)}, \delta_{F}^{(m / 2)}\right]=-\delta_{\{A, F\}}^{(\ell+m) / 2)} \text { for } \ell=\text { odd }} \\
\left\{\delta_{\mathcal{F}_{1}}^{(\ell / 2)}, \delta_{\mathcal{F}_{2}}^{(m / 2)}\right\}= \pm \delta_{\left\{\mathcal{F}_{1}, \mathcal{F}_{2}\right\}}^{(\ell+m) / 2)} \text { for }(\ell, m)=(\text { odd }, \text { odd }) /(\text { even }, \text { even }) \\
\left\{\delta_{\mathcal{F}_{1}}^{(\ell / 2)}, \delta_{\mathcal{F}_{2}}^{(m / 2)}\right\}= \pm \delta_{\left[\mathcal{F}_{1}, \mathcal{F}_{2}\right]}^{(\ell+2)} \text { for }(\ell, m)=(\text { odd }, \text { even }) /(\text { even }, \text { odd })
\end{gathered}
$$


Recall that $\mathcal{A}^{(n)}, \mathcal{A}_{1,2}^{(n)}$ and $\mathcal{F}^{(n)}, \mathcal{F}_{1,2}^{(n)}$ are constant graded matrices of the form (68)-(69).

From (73)-(74) we find that:

$$
\delta_{\mathcal{A}=\mathbb{1}}^{(n)}=-\frac{\partial}{\partial t_{n}} \quad, \quad \delta_{\mathcal{F}=\mathbb{1}}^{\left(n-\frac{1}{2}\right)}=-D_{n}
$$

are (upto an overall minus sign) the superspace isospectral flows of the corresponding $S K P_{\left(R ; M_{B}, M_{F}\right)}$ hierarchy, where the fermionic isospectral flows $D_{n}$ carry the relevant modification (see Eqs.(25)(26)) found in ref. 14 in order to preserve the specific constrained form of (24).

Relations (80)-(83) show that the algebra of symmetry flows (75) for fermionic $\operatorname{SKP}_{\left(R ; M_{B}, M_{F}\right)}$ hierarchy (24) (with $R=2 r+1, M \equiv M_{B}+M_{F}=2 N+1$ ), which contains also Manin-Radul isospectral flows according to (84), spans $\left(\widehat{G L}\left(M_{B}, M_{F}\right)\right)_{+}$- the positive grade part of superloop superalgebra $\widehat{G L}\left(M_{B}, M_{F}\right)$ with half-integer grading $(70)-(72)$.

It is also instructive to rewrite the definitions (66)-(67) and the flow Eqs.(76) $-(79)$ using the short-hand notations (54) for the pertinent (adjoint) super-eigenfuntions:

$$
\begin{gathered}
\mathcal{M}_{\mathcal{A}}^{(\ell / 2)} \equiv \sum_{i, j=1}^{M} \mathcal{A}_{i j}^{(\ell / 2)} \sum_{s=0}^{\ell-1}(-1)^{s(|j|+\ell)} \Phi_{j}^{(\ell-1-s) / 2} \mathcal{D}^{-1} \Psi_{i}^{(s / 2)} \\
\mathcal{M}_{\mathcal{F}}^{(\ell / 2)} \equiv \sum_{i, j=1}^{M} \mathcal{F}_{i j}^{(\ell / 2)} \sum_{s=0}^{\ell-1}(-1)^{s(|j|+\ell)} \Phi_{j}^{(\ell-1-s) / 2} \mathcal{D}^{-1} \Psi_{i}^{(s / 2)} \\
\delta_{\mathcal{A}}^{(\ell / 2)} \Phi_{i}^{(m / 2)}=\mathcal{M}_{\mathcal{A}}^{(\ell / 2)}\left(\Phi_{i}^{(m / 2)}\right)-\sum_{j=1}^{M} \mathcal{A}_{i j}^{(\ell / 2)} \Phi_{j}^{(\ell+m) / 2)} \\
\delta_{\mathcal{A}}^{(\ell / 2)} \Psi_{i}^{(m / 2)}=-\left(\mathcal{M}_{\mathcal{A}}^{(\ell / 2)}\right)^{*}\left(\Psi_{i}^{(m / 2)}\right)+\sum_{j=1}^{M}(-1)^{\ell(|j|+m-1)} \mathcal{A}_{j i}^{(\ell / 2)} \Psi_{j}^{((\ell+m) / 2)} \\
\delta_{\mathcal{F}}^{(\ell / 2)} \Phi_{i}^{(m / 2)}=\mathcal{M}_{\mathcal{F}}^{(\ell / 2)}\left(\Phi_{i}^{(m / 2)}\right)+(-1)^{m-1} \sum_{j=1}^{M} \mathcal{F}_{i j}^{(\ell / 2)} \Phi_{j}^{((\ell+m) / 2)} \\
\delta_{\mathcal{F}}^{(\ell / 2)} \Psi_{i}^{(m / 2)}=-\left(\mathcal{M}_{\mathcal{F}}^{(\ell / 2)}\right)^{*}\left(\Psi_{i}^{(m / 2)}\right)-\sum_{j=1}^{M}(-1)^{(\ell+1)(|j|+m-1)} \mathcal{F}_{j i}^{(\ell / 2)} \Psi_{j}^{((\ell+m) / 2)}
\end{gathered}
$$

Then, the construction of positive-grade superloop superalgebra symmetries of this Section can be straightforwardly carried over to the case of the general unconstrained MR-SKP hierarchy (四). In the latter case all pertinent (adjoint) super-eigenfunctions $\left\{\Phi_{i}^{\ell / 2}, \Psi_{i}^{\ell / 2}\right\}_{i=1, \ldots, M}^{\ell=0,1,2, \ldots}$ are arbitrary, i.e., not related to a finite subset of them unlike (54) and, moreover, their respective numbers $M_{B, F}$ $\left(M=M_{B}+M_{F}\right)$ are also arbitrary. Therefore, the general unconstrained MR-SKP hierarchy possesses $\left(\widehat{G L}\left(M_{B}, M_{F}\right)\right)_{+}$superloop superalgebra symmetries for any $M_{B, F}$.

Concluding this Section, let us also write down the $\delta_{\mathcal{A}, \mathcal{F}}^{(\ell / 2)}$-flow equations for the (adjoint) supereigenfunctions (53) entering the inverse powers of $\mathcal{L}$, which result from consistency of the flow actions (75) with the specific constrained form of $\mathcal{L}^{-K}$ (51) :

$$
\delta_{\mathcal{A}, \mathcal{F}}^{(\ell / 2)} \phi_{I}^{(-m / 2)}=\mathcal{M}_{\mathcal{A}, \mathcal{F}}^{(\ell / 2)}\left(\phi_{I}^{(-m / 2)}\right) \quad, \quad \delta_{\mathcal{A}, \mathcal{F}}^{(\ell / 2)} \psi_{I}^{(-m / 2)}=-\left(\mathcal{M}_{\mathcal{A}, \mathcal{F}}^{(\ell / 2)}\right)^{*}\left(\psi_{I}^{(-m / 2)}\right)
$$




\section{Superloop Superalgebra Symmetries of Constrained SKP Hierarchies: The Case of Bosonic Super-Lax Operators}

Now we will extend the construction of superloop superalgebra additional symmetries from the previous Section to the case of bosonic super-Lax operators $\mathcal{L} \equiv L(33)$.

We find for the counterparts of (66) $-(67)$ the following expressions:

$$
\begin{gathered}
\mathcal{M}_{\mathcal{A}}^{(n)}=\sum_{a, a^{\prime}=1}^{M_{B}} A_{a a^{\prime}}^{(n)} \sum_{k=0}^{n-1} L^{n-k-1}\left(\Phi_{a}^{\prime}\right) \mathcal{D}^{-1}\left(L^{k}\right)^{*}\left(\widetilde{\Psi}_{a}\right)+\sum_{b, b^{\prime}=1}^{M_{F}} D_{b b^{\prime}}^{(n)} \sum_{k=0}^{n-1} L^{n-k-1}\left(\widetilde{\Phi}_{b}^{\prime}\right) \mathcal{D}^{-1}\left(L^{k}\right)^{*}\left(\Psi_{b}\right) \\
\mathcal{M}_{\mathcal{F}}^{\left(n-\frac{1}{2}\right)}=-\sum_{a, b=1}^{M_{B}, M_{F}} B_{a b}^{\left(n-\frac{1}{2}\right)} \sum_{l=0}^{n-2} L^{n-l-2}\left(\widetilde{\Phi}_{b}\right) \mathcal{D}^{-1}\left(L^{l}\right)^{*}\left(\widetilde{\Psi}_{a}\right)+ \\
\quad+\sum_{a, b=1}^{M_{B}, M_{F}} C_{b a}^{\left(n-\frac{1}{2}\right)} \sum_{k=0}^{n-1} L^{n-k-1}\left(\Phi_{a}\right) \mathcal{D}^{-1}\left(L^{k}\right)^{*}\left(\Psi_{b}\right)
\end{gathered}
$$

Here $\mathcal{A}^{(n)}$ and $\mathcal{F}^{\left(n-\frac{1}{2}\right)}$ are constant supermatrices which are purely bosonic and purely fermionic elements belonging to the superloop superalgebra $\widehat{G}\left(M_{B}, M_{F}\right)(70)-(\sqrt[72]{ })$ with grades $n$ and $n-\frac{1}{2}$, respectively:

$$
\mathcal{A}^{(n)}=\left(\begin{array}{cc}
A^{(n)} & 0 \\
0 & D^{(n)}
\end{array}\right) \quad, \quad \mathcal{F}^{\left(n-\frac{1}{2}\right)}=\left(\begin{array}{cc}
0 & B^{\left(n-\frac{1}{2}\right)} \\
C^{\left(n-\frac{1}{2}\right)} & 0
\end{array}\right)
$$

(compare expressions (94) for bosonic super-Lax operators with expressions (68)- 69) for fermionic super-Lax operators).

In particular, we note that:

$$
\mathcal{M}_{\mathcal{A}=\mathbb{1}}^{(n)}=\sum_{a=1}^{N} \sum_{k=0}^{n-1}\left[L^{n-k-1}\left(\widetilde{\Phi}_{a}\right) \mathcal{D}^{-1}\left(L^{k}\right)^{*}\left(\Psi_{a}\right)+L^{n-k-1}\left(\Phi_{a}\right) \mathcal{D}^{-1}\left(L^{k}\right)^{*}\left(\widetilde{\Psi}_{a}\right)\right]=\left(L^{n}\right)_{-}
$$

In full analogy with Eqs. (75)-(79) we construct the following infinite set of bosonic and fermionic flows acting on the bosonic constrained SKP Lax operator $\mathcal{L} \equiv L$ :

$$
\delta_{A}^{(n)} L=\left[\mathcal{M}_{A}^{(n)}, L\right] \quad, \quad \delta_{F}^{\left(n-\frac{1}{2}\right)} L=\left[\mathcal{M}_{F}^{\left(n-\frac{1}{2}\right)}, L\right]
$$

Consistency of the flow actions (96) with the specific constrained form of $\mathcal{L} \equiv L(33)$ implies the following flow actions on the associated (adjoint) super-eigenfunctions:

$$
\begin{gathered}
\delta_{\mathcal{A}}^{(n)} \Phi_{a}=\mathcal{M}_{\mathcal{A}}^{(n)}\left(\Phi_{a}\right)-\sum_{a^{\prime}=1}^{M_{B}} A_{a a^{\prime}}^{(n)} L^{n}\left(\Phi_{a^{\prime}}\right) \quad, \quad \delta_{\mathcal{A}}^{(n)} \Psi_{b}=-\left(\mathcal{M}_{\mathcal{A}}^{(n)}\right)^{*}\left(\Psi_{b}\right)+\sum_{b^{\prime}=1}^{M_{F}} D_{b^{\prime} b}^{(n)}\left(L^{n}\right)^{*}\left(\Psi_{b^{\prime}}\right) \\
\delta_{\mathcal{A}}^{(n)} \widetilde{\Phi}_{b}=\mathcal{M}_{\mathcal{A}}^{(n)}\left(\widetilde{\Phi}_{b}\right)-\sum_{b^{\prime}=1}^{M_{F}} D_{b b^{\prime}}^{(n)} L^{n}\left(\widetilde{\Phi}_{b^{\prime}}\right) \quad, \quad \delta_{\mathcal{A}}^{(n)} \widetilde{\Psi}_{a}=-\left(\mathcal{M}_{\mathcal{A}}^{(n)}\right)^{*}\left(\widetilde{\Psi}_{a}\right)+\sum_{a^{\prime}=1}^{M_{B}} A_{a^{\prime} a}^{(n)}\left(L^{n}\right)^{*}\left(\widetilde{\Psi}_{a^{\prime}}\right) \\
\delta_{\mathcal{F}}^{\left(n-\frac{1}{2}\right)} \Phi_{a}=\mathcal{M}_{\mathcal{F}}^{\left(n-\frac{1}{2}\right)}\left(\Phi_{a}\right)+\sum_{b^{\prime}=1}^{M_{F}} B_{a b^{\prime}}^{\left(n-\frac{1}{2}\right)} L^{n-1}\left(\widetilde{\Phi}_{b^{\prime}}\right)
\end{gathered}
$$




$$
\begin{gathered}
\delta_{\mathcal{F}}^{\left(n-\frac{1}{2}\right)} \Psi_{b}=-\left(\mathcal{M}_{\mathcal{F}}^{\left(n-\frac{1}{2}\right)}\right)^{*}\left(\Psi_{b}\right)-\sum_{a^{\prime}=1}^{M_{B}} B_{a^{\prime} b}^{\left(n-\frac{1}{2}\right)}\left(L^{n-1}\right)^{*}\left(\widetilde{\Psi}_{a^{\prime}}\right) \\
\delta_{\mathcal{F}}^{\left(n-\frac{1}{2}\right)} \widetilde{\Phi}_{b}=\mathcal{M}_{\mathcal{F}}^{\left(n-\frac{1}{2}\right)}\left(\widetilde{\Phi}_{b}\right)-\sum_{a^{\prime}=1}^{M_{B}} C_{b a^{\prime}}^{\left(n-\frac{1}{2}\right)} L^{n}\left(\Phi_{a^{\prime}}\right) \\
\delta_{\mathcal{F}}^{\left(n-\frac{1}{2}\right)} \widetilde{\Psi}_{a}=-\left(\mathcal{M}_{\mathcal{F}}^{\left(n-\frac{1}{2}\right)}\right)^{*}\left(\widetilde{\Psi}_{a}\right)-\sum_{b^{\prime}=1}^{M_{F}} C_{b^{\prime} a}^{\left(n-\frac{1}{2}\right)}\left(L^{n}\right)^{*}\left(\Psi_{b^{\prime}}\right)
\end{gathered}
$$

Now, employing identities (31) we find that the symmetry generating super-pseudo-differential operators (92)-(93) for bosonic constrained super-KP hierarchies satisfy the same type of commutation relations as relations (80)-(82) in the case of fermionic constrained super-KP hierarchies upon replacing there the constant supermatrices of the form (68)-(69) with the corresponding constant supermatrices (94):

$$
\begin{gathered}
\delta_{\mathcal{A}_{1}}^{(n)} \mathcal{M}_{\mathcal{A}_{2}}^{(m)}-\delta_{\mathcal{A}_{2}}^{(m)} \mathcal{M}_{\mathcal{A}_{1}}^{(n)}-\left[\mathcal{M}_{\mathcal{A}_{1}}^{(n)}, \mathcal{M}_{\mathcal{A}_{2}}^{(m)}\right]=\mathcal{M}_{\left[\mathcal{A}_{1}, \mathcal{A}_{2}\right]}^{(n+m)} \\
\delta_{\mathcal{A}}^{(n)} \mathcal{M}_{\mathcal{F}}^{\left(m-\frac{1}{2}\right)}-\delta_{\mathcal{F}}^{\left(m-\frac{1}{2}\right)} \mathcal{M}_{\mathcal{A}}^{(n)}-\left[\mathcal{M}_{\mathcal{A}}^{(n)}, \mathcal{M}_{\mathcal{F}}^{\left(m-\frac{1}{2}\right)}\right]=\mathcal{M}_{[\mathcal{A}, \mathcal{F}]}^{\left(n+m-\frac{1}{2}\right)} \\
\delta_{\mathcal{F}_{1}}^{\left(n-\frac{1}{2}\right)} \mathcal{M}_{\mathcal{F}_{2}}^{\left(m-\frac{1}{2}\right)}+\delta_{\mathcal{F}_{2}}^{\left(m-\frac{1}{2}\right)} \mathcal{M}_{\mathcal{F}_{1}}^{\left(n-\frac{1}{2}\right)}-\left\{\mathcal{M}_{\mathcal{F}_{1}}^{\left(n-\frac{1}{2}\right)}, \mathcal{M}_{\mathcal{F}_{2}}^{\left(m-\frac{1}{2}\right)}\right\}=\mathcal{M}_{\left\{\mathcal{F}_{1}, \mathcal{F}_{2}\right\}}^{(n+m-1)}
\end{gathered}
$$

Therefore, the pertinent flows $\delta_{\mathcal{A}}^{(n)}$ and $\delta_{\mathcal{F}}^{\left(n-\frac{1}{2}\right)}(196)-(102)$ span the following infinite-dimensional superalgebra:

$$
\left[\delta_{\mathcal{A}_{1}}^{(n)}, \delta_{\mathcal{A}_{2}}^{(m)}\right]=\delta_{\left[\mathcal{A}_{1}, \mathcal{A}_{2}\right]}^{(n+m)} \quad, \quad\left[\delta_{\mathcal{A}}^{(n)}, \delta_{\mathcal{F}}^{\left(m-\frac{1}{2}\right)}\right]=\delta_{[\mathcal{A}, \mathcal{F}]}^{\left(n+m-\frac{1}{2}\right)} \quad, \quad\left\{\delta_{\mathcal{F}_{1}}^{\left(n-\frac{1}{2}\right)}, \delta_{\mathcal{F}_{2}}^{\left(m-\frac{1}{2}\right)}\right\}=\delta_{\left\{\mathcal{F}_{1}, \mathcal{F}_{2}\right\}}^{(n+m-1)}
$$

which we denote as $\left(\widehat{G L}_{M_{B}, M F}\right)_{+}$. Similarly to (83) we find that $\left(\widehat{G L}_{M_{B}, M F}\right)_{+}$is the positivegrade part of an infinite-dimensional superalgebra $\widehat{G L}_{M_{B}, M_{F}}$ with half-integer grading consisting of all $\left(M_{B}, M_{F}\right) \times\left(M_{B}, M_{F}\right)$ graded matrices of the form:

$$
\begin{array}{r}
\widehat{G L}_{N_{1}, N_{2}}=\oplus_{\ell \in \mathbb{Z}} G L_{N_{1}, N_{2}}^{(\ell)}, \quad N_{1} \equiv M_{B}, N_{2} \equiv M_{F} \\
G L_{N_{1}, N_{2}}^{(n)}=\left\{\left(\begin{array}{cc}
A^{(n)} & 0 \\
0 & D^{(n)}
\end{array}\right)\right\} \quad, \quad G L_{N_{1}, N_{2}}^{\left(n-\frac{1}{2}\right)}=\left\{\left(\begin{array}{cc}
0 & B^{\left(n-\frac{1}{2}\right)} \\
C^{\left(n-\frac{1}{2}\right)} & 0
\end{array}\right)\right\}
\end{array}
$$

In the present case of bosonic constrained super-KP hierarchies (33) the first relation in (84) is again satisfied, whereas the second relation (84) holds only for fermionic $\operatorname{SKP}_{\left(R ; M_{B}, M_{F}\right)}$ hierarchies.

For later use let us also write down explicitly the $\delta_{\mathcal{A}}^{(n)}$ and $\delta_{\mathcal{F}}^{\left(n-\frac{1}{2}\right)}\left(\left(\widehat{G L}_{M_{B}, M_{F}}\right)_{+}\right.$superloop superalgebra) flow equations for all pertinent (adjoint) super-eigenfunctions (45) :

$$
\begin{array}{ll}
\delta_{\mathcal{A}}^{(n)} \Phi_{a}^{(m)}=\mathcal{M}_{\mathcal{A}}^{(n)}\left(\Phi_{a}^{(m)}\right)-\sum_{a^{\prime}=1}^{M_{B}} A_{a a^{\prime}}^{(n)} \Phi_{a^{\prime}}^{(n+m)} \quad, \quad \delta_{\mathcal{A}}^{(n)} \Psi_{b}^{(m)}=-\left(\mathcal{M}_{\mathcal{A}}^{(n)}\right)^{*}\left(\Psi_{b}^{(m)}\right)+\sum_{b^{\prime}=1}^{M_{F}} D_{b^{\prime} b}^{(n)} \Psi_{b^{\prime}}^{(n+m)} \\
\delta_{\mathcal{A}}^{(n)} \widetilde{\Phi}_{b}^{(m)}=\mathcal{M}_{\mathcal{A}}^{(n)}\left(\widetilde{\Phi}_{b}^{(m)}\right)-\sum_{b^{\prime}=1}^{M_{F}} D_{b b^{\prime}}^{(n)} \widetilde{\Phi}_{b^{\prime}}^{(n+m)} \quad, \quad \delta_{\mathcal{A}}^{(n)} \widetilde{\Psi}_{a}^{(m)}=-\left(\mathcal{M}_{\mathcal{A}}^{(n)}\right)^{*}\left(\widetilde{\Psi}_{a}^{(m)}\right)+\sum_{b^{\prime}=1}^{M_{B}} A_{a^{\prime} a}^{(n)} \widetilde{\Psi}_{a^{\prime}}^{(n+m)}
\end{array}
$$




$$
\begin{gathered}
\delta_{\mathcal{F}}^{\left(n-\frac{1}{2}\right)} \Phi_{a}^{(m)}=\mathcal{M}_{\mathcal{F}}^{\left(n-\frac{1}{2}\right)}\left(\Phi_{a}^{(m)}\right)+\sum_{b^{\prime}=1}^{M_{F}} B_{a b^{\prime}}^{\left(n-\frac{1}{2}\right)} \widetilde{\Phi}_{b^{\prime}}^{(n+m-1)} \\
\delta_{\mathcal{F}}^{\left(n-\frac{1}{2}\right)} \Psi_{b}^{(m)}=-\left(\mathcal{M}_{\mathcal{F}}^{\left(n-\frac{1}{2}\right)}\right)^{*}\left(\Psi_{b}^{(m)}\right)-\sum_{a^{\prime}=1}^{M_{B}} B_{a^{\prime} b}^{\left(n-\frac{1}{2}\right)} \widetilde{\Psi}_{a^{\prime}}^{(n+m-1)} \\
\delta_{\mathcal{F}}^{\left(n-\frac{1}{2}\right)} \widetilde{\Phi}_{b}^{(m)}=\mathcal{M}_{\mathcal{F}}^{\left(n-\frac{1}{2}\right)}\left(\widetilde{\Phi}_{b}^{(m)}\right)-\sum_{a^{\prime}=1}^{M_{B}} C_{b a^{\prime}}^{\left(n-\frac{1}{2}\right)} \Phi_{a^{\prime}}^{(n+m)} \\
\delta_{\mathcal{F}}^{\left(n-\frac{1}{2}\right)} \widetilde{\Psi}_{a}^{(m)}=-\left(\mathcal{M}_{\mathcal{F}}^{\left(n-\frac{1}{2}\right)}\right)^{*}\left(\widetilde{\Psi}_{a}^{(m)}\right)-\sum_{b^{\prime}=1}^{M_{F}} C_{b^{\prime} a}^{\left(n-\frac{1}{2}\right)} \Psi_{b^{\prime}}^{(n+m)}
\end{gathered}
$$

which generalize Eqs. (97)-(102) and where relations (47) have been taken into account. Accordingly, the consistency conditions for the flow Eqs.(96) written in terms of $L^{-K}$ with the specific super-pseudodifferential form of the latter (43) imply for (41)-(42) the following $\left(\widehat{G L}_{M_{B}, M_{F}}\right)_{+}$ superloop superalgebra flow equations:

$$
\begin{aligned}
& \delta_{\mathcal{A}}^{(n)} \stackrel{(\sim)}{\Phi}_{\beta}^{(-m)}=\mathcal{M}_{\mathcal{A}}^{(n)}\left(\stackrel{(\sim)}{\Phi}_{\beta}^{(-m)}\right) \quad, \quad \delta_{\mathcal{A}}^{(n)} \stackrel{(\sim)}{\Psi}_{\beta}^{(-m)}=-\left(\mathcal{M}_{\mathcal{A}}^{(n)}\right) *\left(\stackrel{(\sim)}{\Psi}_{\beta}^{(-m)}\right) \\
& \delta_{\mathcal{F}}^{\left(n-\frac{1}{2}\right)} \stackrel{(\sim)}{\Phi}_{\beta}^{(-m)}=\mathcal{M}_{\mathcal{F}}^{\left(n-\frac{1}{2}\right)}\left(\stackrel{\left(\sim^{\prime}\right.}{\Phi_{\beta}^{(-m)}}\right) \quad, \quad \delta_{\mathcal{F}}^{\left(n-\frac{1}{2}\right)} \stackrel{(\sim)}{\Psi}_{\beta}^{(-m)}=-\left(\mathcal{M}_{\mathcal{F}}^{\left(n-\frac{1}{2}\right)}\right)^{*}\left(\stackrel{(\sim)}{\Psi}_{\beta}^{(-m)}\right)
\end{aligned}
$$

where again relations (47) have been accounted for.

\section{Multi-Component (Matrix) SKP Hierarchies: Supersymmetric Extension of Davey- Stewartson System}

Let us now consider the following subalgebra of the superloop superalgebra symmetry flows for bosonic $\operatorname{SKP}_{(2 r ; N, N)}$ hierarchies (i.e., $R=2 r, M_{B}=M_{F}=N$; cf. (92)-(93) and (96)) which are defined as:

$$
\begin{aligned}
& \delta_{\mathcal{A}=\mathcal{E}_{k}}^{(n)} \equiv-\partial / \partial \stackrel{(k)}{t_{n}}, \quad \delta_{\mathcal{F}=\mathcal{E}_{k}}^{\left(n-\frac{1}{2}\right)} \equiv-\stackrel{(k)}{D_{n}} \\
& \mathcal{E}_{k}^{(n)}=\left(\begin{array}{cc}
E_{k} & 0 \\
0 & E_{k}
\end{array}\right) \quad, \quad \mathcal{E}_{k}^{\left(n-\frac{1}{2}\right)}=\left(\begin{array}{cc}
0 & E_{k} \\
E_{k} & 0
\end{array}\right) \quad \text { with } E_{k} \equiv \operatorname{diag}(0, \ldots, 0, \stackrel{(k)}{1}, 0, \ldots, 0)
\end{aligned}
$$

where $k=1, \ldots, N$. The flows (116) span a direct sum of $N$ copies of the original Manin-Radul isospectral flow algebra (12):

$$
\left\{\stackrel{(k)}{D}_{n}, \stackrel{(l)}{D}_{m}\right\}=-\delta_{k l} \partial / \partial^{(k)}{ }_{n+m-1} \quad, \quad \text { rest }=0 \quad ; \quad k, l=1, \ldots, N, n, m=1,2, \ldots
$$

which justifies their representation in a form similar to (12):

$$
\stackrel{(k)}{D_{n}}=\partial / \partial \stackrel{(k)}{\theta}_{n}-\sum_{s=1}^{\infty} \stackrel{(k)}{\theta}_{s} \partial / \partial \stackrel{(k)}{t}_{n+s-1}
$$

Now, we can construct the following supersymmetric extended integrable hierarchy built on the original bosonic $\operatorname{SKP}_{(2 r ; N, N)}$ supersymmetric hierarchy (33) by supplementing the latter with 
the set of additional superloop superalgebra supersymmetric Manin-Radul-like flows (116)-(119) (recall here $\left.L \equiv \mathcal{L}_{(2 r ; N, N)}(33)\right)$ :

$$
\partial / \partial{ }_{t}{ }_{n} L=-\left[\mathcal{M}_{k}^{(n)}, L\right] \quad, \quad \stackrel{(k)}{D_{n}} L=-\left[\mathcal{M}_{k}^{\left(n-\frac{1}{2}\right)}, L\right]
$$

with:

$$
\begin{aligned}
\mathcal{M}_{k}^{(n)} & \equiv \sum_{s=0}^{n-1}\left[L^{n-s-1}\left(\widetilde{\Phi}_{k}\right) \mathcal{D}^{-1}\left(L^{s}\right)^{*}\left(\Psi_{k}\right)+L^{n-s-1}\left(\Phi_{k}\right) \mathcal{D}^{-1}\left(L^{s}\right)^{*}\left(\widetilde{\Psi}_{k}\right)\right] \\
\mathcal{M}_{k}^{\left(n-\frac{1}{2}\right)} & \equiv \sum_{s=0}^{n-1} L^{n-s-1}\left(\Phi_{k}\right) \mathcal{D}^{-1}\left(L^{s}\right)^{*}\left(\Psi_{k}\right)-\sum_{s=0}^{n-2} L^{n-s-2}\left(\widetilde{\Phi}_{k}\right) \mathcal{D}^{-1}\left(L^{s}\right)^{*}\left(\widetilde{\Psi}_{k}\right)
\end{aligned}
$$

where the flow action on the constituent (adjoint) super-eigenfunctions is given by (cf. Eqs.97)(102)) :

$$
\begin{aligned}
& \partial / \partial \stackrel{(k)}{t}_{n} \stackrel{(\sim)}{\Phi_{a}}=-\mathcal{M}_{k}^{(n)}\left(\stackrel{(\sim)}{\Phi_{a}}\right) \quad, \quad \partial / \partial \stackrel{(k)}{t} \stackrel{(\sim)}{\Psi}_{a}=\left(\mathcal{M}_{k}^{(n)}\right)^{*}\left(\stackrel{(\sim)}{\Psi_{a}}\right) \quad, \quad a \neq k \\
& \partial / \partial \stackrel{(k)}{t}_{n} \stackrel{(\sim)}{\Phi_{k}}=-\mathcal{M}_{k}^{(n)}\left(\stackrel{(\sim)}{\Phi_{k}}\right)+L^{n}\left(\stackrel{(\sim)}{\Phi}_{k}\right) \quad, \quad \partial / \partial \stackrel{(k)}{t}_{n}{\stackrel{(\sim)}{\Psi_{k}}}_{k}=\left(\mathcal{M}_{k}^{(n)}\right)^{*}\left(\stackrel{(\sim)}{\Psi}_{k}\right)-\left(L^{n}\right)^{*}\left(\stackrel{(\sim)}{\Phi}_{k}\right)
\end{aligned}
$$

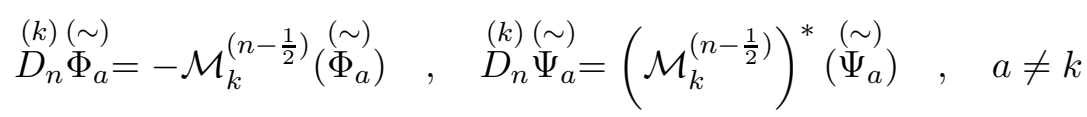

$$
\begin{aligned}
& \stackrel{(k)}{D_{n}} \Phi_{k}=-\mathcal{M}_{k}^{\left(n-\frac{1}{2}\right)}\left(\Phi_{k}\right)+L^{n-1}\left(\widetilde{\Phi}_{k}\right) \quad, \quad \stackrel{(k)}{D_{n}} \Psi_{k}=\left(\mathcal{M}_{k}^{\left(n-\frac{1}{2}\right)}\right)^{*}\left(\Psi_{k}\right)+\left(L^{n-1}\right)^{*}\left(\widetilde{\Psi}_{k}\right) \\
& \stackrel{(k)}{D_{n}} \widetilde{\Phi}_{k}=-\mathcal{M}_{k}^{\left(n-\frac{1}{2}\right)}\left(\widetilde{\Phi}_{k}\right)+L^{n}\left(\Phi_{k}\right) \quad, \quad{\stackrel{(k)}{D_{n}}}^{\Psi} \widetilde{\Psi}_{k}=\left(\mathcal{M}_{k}^{\left(n-\frac{1}{2}\right)}\right)^{*}\left(\widetilde{\Psi}_{k}\right)+\left(L^{n}\right)^{*}\left(\Psi_{k}\right)
\end{aligned}
$$

The above construction is the superspace analog of our construction in refs. [25, 26], where the corresponding ordinary bosonic scalar (one-component) KP hierarchy, supplemented with the flows belonging to the Cartan subalgebra of additional loop-algebra symmetries, was identified as a matrix (multi-component) KP hierarchy. Therefore it is natural to call the supersymmetric extended KP hierarchy defined by Eqs.(120)-(127) $N$-component constrained super-KP hierarchy.

It is well-known that ordinary bosonic multi-component KP hierarchies contain various physically interesting nonlinear systems such as 2-dimensional Toda lattice, Davey-Stewartson and $N$ wave resonant systems. As a non-trivial illustration of the properties of the new $N$-component constrained super-KP hierarchies (120)-127) we will show that the $\operatorname{SKP}_{(2 ; 2,2)}$ model contains a supersymmetric version of ordinary Davey-Stewartson system.

Thus, we consider the special case $N=2$ in Eqs. 120)-127). We take for convenience the following two mutually (anti-)commuting infinite sets of Manin-Radul-like flows: $\left\{\partial / \partial t_{n}, D_{n}\right\}$ (the original MR-SKP isospectral flows) and $\left\{\partial / \partial \bar{t}_{n} \equiv-\partial / \partial \stackrel{(1)}{t}_{n}, \stackrel{(1)}{D}_{n}\right\} \quad\left(\right.$ recall $\partial / \partial t_{n}=\partial / \partial t_{1}+\partial / \partial t_{2}^{(1)}$ according to first Eq.(84)). In particular, we will use the short-hand notation $\bar{\partial} \equiv \partial / \partial \bar{t}_{1} \equiv-\partial / \partial t_{1}$. We will need the following explicit expressions (cf. the general notations and relations from Section 2) :

$$
L \equiv L_{(2 ; 2,2)}=\partial+\sum_{a=1}^{2}\left(\widetilde{\Phi}_{a} \mathcal{D}^{-1} \Psi_{a}+\Phi_{a} \mathcal{D}^{-1} \widetilde{\Psi}_{a}\right) \equiv \mathcal{W} \partial \mathcal{W}^{-1} \equiv \partial+u_{\frac{1}{2}} \mathcal{D}^{-1}+u_{1} \mathcal{D}^{-1}+\ldots
$$




$$
\begin{gathered}
u_{\frac{1}{2}}=-\partial \alpha_{1} \equiv \sum_{a=1}^{2}\left(\widetilde{\Phi}_{a} \Psi_{a}-\Phi_{a} \widetilde{\Psi}_{a}\right) \quad, \quad u_{1}=-\beta_{1}-\alpha_{1} \partial \alpha_{1} \equiv \sum_{a=1}^{2}\left(\widetilde{\Phi}_{a} \mathcal{D}_{\theta} \Psi_{a}+\Phi_{a} \mathcal{D}_{\theta} \widetilde{\Psi}_{a}\right) \\
\left(L^{2}\right)_{+}=\partial^{2}+2 u_{\frac{1}{2}} \mathcal{D}+2 u_{1} \quad, \quad\left(L^{2}\right)_{+}^{*}=\partial^{2}+2 u_{\frac{1}{2}} \mathcal{D}+2\left(u_{1}-\mathcal{D}_{\theta} u_{\frac{1}{2}}\right) \\
\mathcal{M}_{1}^{(1)}=\widetilde{\Phi}_{1} \mathcal{D}^{-1} \Psi_{1}+\Phi_{1} \mathcal{D}^{-1} \widetilde{\Psi}_{1} \\
\mathcal{M}_{1}^{(2)}=L\left(\widetilde{\Phi}_{1}\right) \mathcal{D}^{-1} \Psi_{1}+L\left(\Phi_{1}\right) \mathcal{D}^{-1} \widetilde{\Psi}_{1}+\widetilde{\Phi}_{1} \mathcal{D}^{-1} L^{*}\left(\Psi_{1}\right)+\Phi_{1} \mathcal{D}^{-1} L^{*}\left(\widetilde{\Psi}_{1}\right)
\end{gathered}
$$

For the first additional symmetry flow $\bar{\partial}$ we have:

$$
\begin{gathered}
\bar{\partial} \stackrel{(\sim)}{\Phi}_{1}=\mathcal{M}_{1}^{(1)}\left(\stackrel{(\sim)}{\left.\Phi_{1}\right)}-L \stackrel{(\sim)}{\left(\Phi_{1}\right)} \quad, \quad \bar{\partial} \stackrel{(\sim)}{\Psi_{1}}=-\left(\mathcal{M}_{1}^{(1)}\right)^{*}\left(\stackrel{(\sim)}{\Psi_{1}}\right)+L^{*}\left(\stackrel{(\sim)}{\left(\Psi_{1}\right)}\right)\right. \\
\bar{\partial} \alpha_{1}=\widetilde{\Phi}_{1} \Psi_{1}-\Phi_{1} \widetilde{\Psi}_{1} \quad, \quad \bar{\partial} \beta_{1}=\Phi_{1} \mathcal{D}_{\theta} \widetilde{\Psi}_{1}+\widetilde{\Phi}_{1} \mathcal{D}_{\theta} \Psi_{1}+\alpha_{1}\left(\widetilde{\Phi}_{1} \Psi_{1}-\Phi_{1} \widetilde{\Psi}_{1}\right)
\end{gathered}
$$

where the last two Eqs. (134) are obtained from $\bar{\partial} \mathcal{W}=\mathcal{M}_{1}^{(1)} \mathcal{W}$ upon inserting there the expansion for $\mathcal{W}$ (5). Recall also that $\alpha_{1}$ and $\beta_{1}$ are expressed in terms of the constituent (adjoint) supereigenfunctions of the super-Lax operator (128) through Eqs.(129). Further, taking the superresiduum of the superspace operator flow equation $\bar{\partial} L=\left[\mathcal{M}_{1}^{(1)}, L\right]$ we get:

$$
\bar{\partial} u_{\frac{1}{2}}=-\partial\left(\widetilde{\Phi}_{1} \Psi_{1}-\Phi_{1} \widetilde{\Psi}_{1}\right)
$$

We now consider the following system of flow equations:

$$
\left.\left.\frac{\partial}{\partial t_{2}} \stackrel{(\sim)}{\Phi_{1}}=\left(L^{2}\right)_{+} \stackrel{(\sim)}{\left(\Phi_{1}\right.}\right) \quad, \quad \frac{\partial}{\partial t_{2}} \stackrel{(\sim)}{\Psi_{1}}=-\left(L^{2}\right)_{+}^{*} \stackrel{(\sim)}{\left(\Psi_{1}\right.}\right)
$$

which are the standard (adjoint) super-eigenfunction equations (20) for the second isospectral bosonic flow $\partial / \partial t_{2}$ where the super-differential operators on the r.h.s. are given by (130), and:

$$
\partial / \partial \bar{t}_{2} \stackrel{(\sim)}{\Phi_{1}}=\mathcal{M}_{1}^{(2)}\left(\stackrel{(\sim)}{\Phi_{1}}\right)-L^{2}\left(\stackrel{(\sim)}{\left.\Phi_{1}\right)} \quad, \quad \partial / \partial \bar{t}_{2} \stackrel{(\sim)}{\Psi}_{1}=-\left(\mathcal{M}_{1}^{(2)}\right)^{*}\left(\stackrel{(\sim)}{\Psi}_{1}\right)+\left(L^{2}\right)^{*}\left(\stackrel{(\sim)}{\Psi_{1}}\right)\right.
$$

which are the flow equations for the second bosonic additional-symmetry flow $\partial / \partial \bar{t}_{2}$ with $\mathcal{M}_{1}^{(2)}$ as in (132). The explicit form of Eqs.1136-1137) reads:

$$
\begin{gathered}
\frac{\partial}{\partial t_{2}} \Phi_{1}=\left[\partial^{2}+2 u_{\frac{1}{2}} \mathcal{D}+2 u_{1}\right] \Phi_{1} \quad, \quad \partial / \partial \bar{t}_{2} \Phi_{1}=\left[-\bar{\partial}^{2}+2 \bar{\partial}\left(\mathcal{D}_{\theta}^{-1}\left(\Phi_{1} \widetilde{\Psi}_{1}\right)\right)\right] \Phi_{1}-2 \bar{\partial}\left(\mathcal{D}_{\theta}^{-1}\left(\Phi_{1} \Psi_{1}\right)\right) \widetilde{\Phi}_{1} \\
\frac{\partial}{\partial t_{2}} \widetilde{\Phi}_{1}=\left[\partial^{2}+2 u_{\frac{1}{2}} \mathcal{D}+2 u_{1}\right] \widetilde{\Phi}_{1} \quad, \quad \partial / \partial \bar{t}_{2} \widetilde{\Phi}_{1}=\left[-\bar{\partial}^{2}+2 \bar{\partial}\left(\mathcal{D}_{\theta}^{-1}\left(\widetilde{\Phi}_{1} \Psi_{1}\right)\right)\right] \widetilde{\Phi}_{1}-2 \bar{\partial}\left(\mathcal{D}_{\theta}^{-1}\left(\widetilde{\Phi}_{1} \widetilde{\Psi}_{1}\right)\right) \Phi_{1} \\
\frac{\partial}{\partial t_{2}} \Psi_{1}=-\left[\partial^{2}+2 u_{\frac{1}{2}} \mathcal{D}+2\left(u_{1}-\mathcal{D}_{\theta} u_{\frac{1}{2}}\right)\right] \Psi_{1} \\
\partial / \partial \bar{t}_{2} \Psi_{1}=\left[\bar{\partial}^{2}-2 \bar{\partial}\left(\mathcal{D}_{\theta}^{-1}\left(\widetilde{\Phi}_{1} \Psi_{1}\right)\right)\right] \Psi_{1}+2 \bar{\partial}\left(\mathcal{D}_{\theta}^{-1}\left(\Phi_{1} \Psi_{1}\right)\right) \widetilde{\Psi}_{1} \\
\frac{\partial}{\partial t_{2}} \widetilde{\Psi}_{1}=\left[\partial^{2}+2 u_{\frac{1}{2}} \mathcal{D}+2\left(u_{1}-\mathcal{D}_{\theta} u_{\frac{1}{2}}\right)\right] \widetilde{\Psi}_{1} \\
\partial / \partial \bar{t}_{2} \widetilde{\Psi}_{1}=\left[\bar{\partial}^{2}-2 \bar{\partial}\left(\mathcal{D}_{\theta}^{-1}\left(\Phi_{1} \widetilde{\Psi}_{1}\right)\right)\right] \widetilde{\Psi}_{1}-2 \bar{\partial}\left(\mathcal{D}_{\theta}^{-1}\left(\widetilde{\Phi}_{1} \widetilde{\Psi}_{1}\right)\right) \Psi_{1}
\end{gathered}
$$


Introducing a new time variable $T=t_{2}-\bar{t}_{2}$ and subtracting $\partial / \partial \bar{t}_{2}$-flow equations from the corresponding $\partial / \partial t_{2}$-flow equations above, we arrive at the following system of super-differential evolution equations for $\Phi_{1}, \widetilde{\Phi}_{1}, \Psi_{1}, \widetilde{\Psi}_{1}$ regarded as functions of $\left(T, x \equiv t_{1}, y \equiv \bar{t}_{1}, \theta\right)$ (i.e., functions on superspace $\mathbb{R}^{2 \mid 1}$ with coordinates $\left.(x, y, \theta)\right)$ and suppressing the dependence on the rest of the bosonic and ferminionic flow parameters:

$$
\begin{gathered}
\frac{\partial}{\partial T} \Phi_{1}=\left[\frac{1}{2}\left(\partial^{2}+\bar{\partial}^{2}\right)+u_{\frac{1}{2}} \mathcal{D}+\left(\mathcal{G}+2\left(\Phi_{1} \mathcal{D}_{\theta} \widetilde{\Psi}_{1}+\widetilde{\Phi}_{1} \mathcal{D}_{\theta} \Psi_{1}\right)\right)\right] \Phi_{1}+\mathcal{F} \widetilde{\Phi}_{1} \\
\frac{\partial}{\partial T} \widetilde{\Phi}_{1}=\left[\frac{1}{2}\left(\partial^{2}+\bar{\partial}^{2}\right)+u_{\frac{1}{2}} \mathcal{D}+\left(\widetilde{\mathcal{G}}+2\left(\Phi_{1} \mathcal{D}_{\theta} \widetilde{\Psi}_{1}+\widetilde{\Phi}_{1} \mathcal{D}_{\theta} \Psi_{1}\right)\right)\right] \widetilde{\Phi}_{1}+\widetilde{\mathcal{F}} \Phi_{1} \\
\frac{\partial}{\partial T} \Psi_{1}=-\left[\frac{1}{2}\left(\partial^{2}+\bar{\partial}^{2}\right)+u_{\frac{1}{2}} \mathcal{D}+\left(\widetilde{\mathcal{G}}-\mathcal{D}_{\theta} u_{\frac{1}{2}}+2\left(\Phi_{1} \mathcal{D}_{\theta} \widetilde{\Psi}_{1}+\widetilde{\Phi}_{1} \mathcal{D}_{\theta} \Psi_{1}\right)\right)\right] \Psi_{1}-\mathcal{F} \widetilde{\Psi}_{1} \\
\frac{\partial}{\partial T} \widetilde{\Psi}_{1}=-\left[\frac{1}{2}\left(\partial^{2}+\bar{\partial}^{2}\right)+u_{\frac{1}{2}} \mathcal{D}+\left(\mathcal{G}-\mathcal{D}_{\theta} u_{\frac{1}{2}}+2\left(\Phi_{1} \mathcal{D}_{\theta} \widetilde{\Psi}_{1}+\widetilde{\Phi}_{1} \mathcal{D}_{\theta} \Psi_{1}\right)\right)\right] \widetilde{\Psi}_{1}+\widetilde{\mathcal{F}} \Psi_{1}
\end{gathered}
$$

The coefficient super-functions $u_{\frac{1}{2}}, \mathcal{F}, \widetilde{\mathcal{F}}, \mathcal{G}, \widetilde{\mathcal{G}}$ in Eqs. (142)-145) are related with $\Phi_{1}, \widetilde{\Phi}_{1}, \Psi_{1}, \widetilde{\Psi}_{1}$ through non-dynamical super-differential relations as follows:

$$
\begin{gathered}
\bar{\partial} u_{\frac{1}{2}}=-\partial\left(\widetilde{\Phi}_{1} \Psi_{1}-\Phi_{1} \widetilde{\Psi}_{1}\right) \quad, \quad \mathcal{D}_{\theta} \mathcal{F}=\bar{\partial}\left(\Phi_{1} \Psi_{1}\right) \quad, \quad \mathcal{D}_{\theta} \widetilde{\mathcal{F}}=\bar{\partial}\left(\widetilde{\Phi}_{1} \widetilde{\Psi}_{1}\right) \\
\partial \bar{\partial} \mathcal{G}+(\partial+\bar{\partial})^{2}\left(\Phi_{1} \mathcal{D}_{\theta} \widetilde{\Psi}_{1}+\widetilde{\Phi}_{1} \mathcal{D}_{\theta} \Psi_{1}\right)= \\
2 \partial\left(u_{\frac{1}{2}}\left(\widetilde{\Phi}_{1} \Psi_{1}-\Phi_{1} \widetilde{\Psi}_{1}\right)\right)+\bar{\partial}^{2}\left(\widetilde{\Phi}_{1} \mathcal{D}_{\theta} \Psi_{1}+\widetilde{\Psi}_{1} \mathcal{D}_{\theta} \Phi_{1}\right) \\
\mathcal{D}_{\theta}(\mathcal{G}-\widetilde{\mathcal{G}})=\bar{\partial}\left(\widetilde{\Phi}_{1} \Psi_{1}-\Phi_{1} \widetilde{\Psi}_{1}\right)
\end{gathered}
$$

The first relation in (146) coincides with Eq.(135). Relations (147) and (148) result from the definitions of $\mathcal{G}$ and $\widetilde{\mathcal{G}}$ :

$$
\begin{aligned}
& \mathcal{G} \equiv u_{1}-\bar{\partial}\left(\mathcal{D}_{\theta}^{-1}\left(\Phi_{1} \widetilde{\Psi}_{1}\right)\right)-2\left(\Phi_{1} \mathcal{D}_{\theta} \widetilde{\Psi}_{1}+\widetilde{\Phi}_{1} \mathcal{D}_{\theta} \Psi_{1}\right) \\
& \widetilde{\mathcal{G}} \equiv u_{1}-\bar{\partial}\left(\mathcal{D}_{\theta}^{-1}\left(\widetilde{\Phi}_{1} \Psi_{1}\right)\right)-2\left(\Phi_{1} \mathcal{D}_{\theta} \widetilde{\Psi}_{1}+\widetilde{\Phi}_{1} \mathcal{D}_{\theta} \Psi_{1}\right)
\end{aligned}
$$

with $u_{1}$ as in (8) and (129), upon taking into account Eqs.(134).

The system of evolution Eqs. (142)-(145) together with the non-dynamical relations (146)-(148) is the supersymetric extension of the ordinary bosonic Davey-Stewartson system. Indeed, let us take the bosonic limit in (142)-(148), meaning that we set all fermionic component fields in the pertinent superfields equal to zero and in addition we put the fermionic superspace coordinate $\theta=0$. Then, the only surviving functions are:

$$
\phi_{1}=\left.\Phi_{1}\right|_{\theta=0}, \quad \psi_{1}=\left.\mathcal{D}_{\theta} \widetilde{\Psi}_{1}\right|_{\theta=0} \quad, \quad G=\left.\mathcal{G}\right|_{\theta=0}=\left.\widetilde{\mathcal{G}}\right|_{\theta=0}
$$

and the superspace system (142)-(148) reduces to the ordinary bosonic system of nonlinear equations:

$$
\begin{aligned}
\frac{\partial}{\partial T} \phi_{1} & =\left[\frac{1}{2}\left(\partial^{2}+\bar{\partial}^{2}\right)+G+2 \phi_{1} \psi_{1}\right] \phi_{1} \\
\frac{\partial}{\partial T} \psi_{1} & =-\left[\frac{1}{2}\left(\partial^{2}+\bar{\partial}^{2}\right)+G+2 \phi_{1} \psi_{1}\right] \psi_{1}
\end{aligned}
$$




$$
\partial \bar{\partial} G+(\partial+\bar{\partial})^{2}\left(\phi_{1} \psi_{1}\right)=0
$$

which is precisely the standard Davey-Stewartson system.

\section{8. "Negative"-Grade Superloop Superalgebra Symmetries}

\section{1 "Negative"-Grade Symmetries of Bosonic $S K P_{\left(R ; M_{B}, M_{F}\right)}$ Hierarchies}

Using the same technique as in Sections 5 and 6, we can construct a "negative"-grade superloop superalgebra of additional symmetries for constrained super-KP hierarchies (24). First we will consider explicitly the case of $S K P_{\left(R ; M_{B}, M_{F}\right)}$ hierarchies with bosonic super-Lax operators (33).

Following the pattern in Eqs.(92)-(93) we consider the following set of additional symmetry generating super-pseudo-differential operators:

$$
\begin{array}{r}
\mathcal{M}_{\overline{\mathcal{A}}}^{(-n)}=\sum_{a, b=1}^{N+r} \sum_{s=1}^{n}\left[\bar{A}_{a b}^{(-n)} \Phi_{b}^{(-(n-s+1))} \mathcal{D}^{-1} \widetilde{\Psi}_{a}^{(-s)}+\bar{D}_{a b}^{(-n)} \widetilde{\Phi}_{b}^{(-(n-s+1))} \mathcal{D}^{-1} \Psi_{a}^{(-s)}\right] \\
\mathcal{M}_{\overline{\mathcal{F}}}^{\left(-n+\frac{1}{2}\right)}=\sum_{a, b=1}^{N+r} \sum_{s=1}^{n}\left[\bar{C}_{a b}^{\left(-n+\frac{1}{2}\right)} \Phi_{b}^{(-(n-s+1))} \mathcal{D}^{-1} \Psi_{a}^{(-s)}-\bar{B}_{a b}^{\left(-n+\frac{1}{2}\right)} \widetilde{\Phi}_{b}^{(-(n-s))} \mathcal{D}^{-1} \widetilde{\Psi}_{a}^{(-s)}\right]
\end{array}
$$

and their associated supersymmetric flows:

$$
\delta_{\overline{\mathcal{A}}}^{(-n)} L=\left[\mathcal{M}_{\overline{\mathcal{A}}}^{(-n)}, L\right] \quad, \quad \delta_{\overline{\mathcal{F}}}^{\left(-n+\frac{1}{2}\right)} L=\left[\mathcal{M}_{\overline{\mathcal{F}}}^{\left(-n+\frac{1}{2}\right)}, L\right]
$$

where the short-hand notations (41)--(42) have been employed. Here $\overline{\mathcal{A}}^{(-n)}$ and $\bar{F}^{\left(-n-\frac{1}{2}\right)}$ are constant supermatrices - elements of superloop superalgebra $\widehat{G}_{N+r, N+r}$ (cf. (107)) having a form similar to (94):

$$
\overline{\mathcal{A}}^{(-n)}=\left(\begin{array}{cc}
\bar{A}^{(-n)} & 0 \\
0 & \bar{D}^{(-n)}
\end{array}\right) \quad, \quad \mathcal{F}^{\left(-n+\frac{1}{2}\right)}=\left(\begin{array}{cc}
0 & \bar{B}^{\left(-n+\frac{1}{2}\right)} \\
\bar{C}^{\left(-n+\frac{1}{2}\right)} & 0
\end{array}\right)
$$

where now all matrix blocks $\bar{A}^{(-n)}, \bar{B}^{\left(-n+\frac{1}{2}\right)}, \bar{C}^{\left(-n+\frac{1}{2}\right)}, \bar{D}^{(-n)}$ are of size $(N+r) \times(N+r)$. Furthermore, $\overline{\mathcal{A}}^{(-n)} \neq \mathbb{1}$ in Eqs. 155), (157) since $\mathcal{M}_{\overline{\mathcal{A}}=\mathbb{1}}^{(-n)}=L^{-n}$ (cf. Eq.(43)), so that $\delta_{\overline{\mathcal{A}}=\mathbb{1}}^{(-n)}$ does not generate any flow according to first Eq.(157). Recall also, that according to (49) all superfunctions entering the super-pseudo-differential operators (155)-(156) are again (adjoint) supereigenfunctions of the bosonic $\operatorname{SKP}_{\left(R ; M_{B}, M_{F}\right)}$ hierarchy (33).

Consistency of the flows action (157) with the constrained form (33) of $L$ and its inverse (40) (or (43)) requires that the "negative-grade" flows act on the pertinent (adjoint) super-eigenfunctions as follows:

$$
\begin{aligned}
& \delta_{\overline{\mathcal{A}}}^{(-n)} \stackrel{(\sim)}{\Phi_{a}^{(m)}}=\mathcal{M}_{\overline{\mathcal{A}}}^{(-n)}\left(\stackrel{(\sim)}{\Phi_{a}^{(m)}}\right) \quad, \quad \delta_{\overline{\mathcal{A}}}^{(-n)} \stackrel{(\sim)}{\Psi}_{a}^{(m)}=-\left(\mathcal{M}_{\overline{\mathcal{A}}}^{(-n)}\right)^{*}\left(\stackrel{(\sim)}{\Psi}_{a}^{(m)}\right) \\
& \delta_{\overline{\mathcal{F}}}^{\left(-n+\frac{1}{2}\right)} \stackrel{(\sim)}{\Phi}_{a}^{(m)}=\mathcal{M}_{\overline{\mathcal{F}}}^{\left(-n+\frac{1}{2}\right)}\left(\stackrel{(\sim)}{\Phi_{a}^{(m)}}\right) \quad, \quad \delta_{\overline{\mathcal{F}}}^{\left(-n+\frac{1}{2}\right)} \stackrel{(\sim)}{\Psi}_{a}^{(m)}=-\left(\mathcal{M}_{\overline{\mathcal{F}}}^{\left(-n+\frac{1}{2}\right)}\right)^{*}\left(\stackrel{(\sim)}{\Psi}_{a}^{(m)}\right) \\
& \delta_{\overline{\mathcal{A}}}^{(-n)} \stackrel{(\sim)}{\Phi}_{a}^{(-m)}=\mathcal{M}_{\overline{\mathcal{A}}}^{(-n)}\left(\stackrel{(\sim)}{\Phi_{a}^{(-m)}}\right)-\sum_{b=1}^{N+r} \bar{A}_{a b}^{(-n)}{\stackrel{(\sim)}{\Phi_{b}^{(}}}^{(-n-m)} \\
& \delta_{\overline{\mathcal{A}}}^{(-n)} \stackrel{(\widetilde{\sim}}{\Psi}_{a}^{(-m)}=-\left(\mathcal{M}_{\overline{\mathcal{A}}}^{(-n)}\right)^{*}\left(\stackrel{(\widetilde{\Psi}}{\Psi}_{a}^{(-m)}\right)+\sum_{b=1}^{N+r} \bar{D}_{b a}^{(-n)} \stackrel{(\sim)}{\Psi}_{b}^{(-n-m)}
\end{aligned}
$$




$$
\begin{aligned}
& \delta_{\overline{\mathcal{A}}}^{(-n)} \stackrel{\left.(\sim)^{(}\right)}{(-m)}=\mathcal{M}_{\overline{\mathcal{A}}}^{(-n)}\left(\stackrel{(\sim)}{\Phi_{a}^{(-m)}}\right)-\sum_{b=1}^{N+r} \bar{D}_{a b}^{(-n)}{\stackrel{(\sim)}{\left.\Phi_{b}^{(}-n-m\right)}}^{(-n)} \\
& \delta_{\overline{\mathcal{A}}}^{(-n)} \stackrel{(\sim)}{\Psi}_{a}^{(-m)}=-\left(\mathcal{M}_{\overline{\mathcal{A}}}^{(-n)}\right)^{*}\left(\stackrel{(\sim)}{\Psi}_{a}^{(-m)}\right)+\sum_{b=1}^{N+r} \bar{A}_{b a}^{(-n)} \stackrel{(\sim)}{\Psi}_{b}^{(-n-m)} \\
& \delta_{\overline{\mathcal{F}}}^{\left(-n+\frac{1}{2}\right)} \Phi_{a}^{(-m)}=\mathcal{M}_{\overline{\mathcal{F}}}^{\left(-n+\frac{1}{2}\right)}\left(\Phi_{a}^{(-m)}\right)+\sum_{b=1}^{N+r} \bar{B}_{a b}^{\left(-n+\frac{1}{2}\right)} \widetilde{\Phi}_{b}^{(-n-m+1)} \\
& \delta_{\overline{\mathcal{F}}}^{\left(-n+\frac{1}{2}\right)} \Psi_{a}^{(-m)}=-\left(\mathcal{M}_{\overline{\mathcal{F}}}^{\left(-n+\frac{1}{2}\right)}\right)^{*}\left(\Psi_{a}^{(-m)}\right)-\sum_{b=1}^{N+r} \bar{B}_{b a}^{\left(-n+\frac{1}{2}\right)} \widetilde{\Psi}_{b}^{(-n-m+1)} \\
& \delta_{\overline{\mathcal{F}}}^{\left(-n+\frac{1}{2}\right)} \widetilde{\Phi}_{a}^{(-m)}=\mathcal{M}_{\overline{\mathcal{F}}}^{\left(-n+\frac{1}{2}\right)}\left(\widetilde{\Phi}_{a}^{(-m)}\right)-\sum_{b=1}^{N+r} \bar{C}_{a b}^{\left(-n+\frac{1}{2}\right)} \Phi_{b}^{(-n-m)} \\
& \delta_{\overline{\mathcal{F}}}^{\left(-n+\frac{1}{2}\right)} \widetilde{\Psi}_{a}^{(-m)}=-\left(\mathcal{M}_{\overline{\mathcal{F}}}^{\left(-n+\frac{1}{2}\right)}\right)^{*}\left(\widetilde{\Psi}_{a}^{(-m)}\right)-\sum_{b=1}^{N+r} \bar{C}_{b a}^{\left(-n+\frac{1}{2}\right)} \Psi_{b}^{(-n-m)}
\end{aligned}
$$

Following the same steps as in the derivation of (80)-(83) and (106) we obtain:

$$
\begin{array}{r}
\delta_{\overline{\mathcal{A}}_{1}}^{(-n)} \mathcal{M}_{\overline{\mathcal{A}}_{2}}^{(-m)}-\delta_{\overline{\mathcal{A}}_{2}}^{(-m)} \mathcal{M}_{\overline{\mathcal{A}}_{1}}^{(-n)}-\left[\mathcal{M}_{\overline{\mathcal{A}}_{1}}^{(-n)}, \mathcal{M}_{\overline{\mathcal{A}}_{2}}^{(-m)}\right]=\mathcal{M}_{\left[\overline{\mathcal{A}}_{1}, \overline{\mathcal{A}}_{2}\right]}^{(-n-m)} \\
\delta_{\overline{\mathcal{A}}}^{(-n)} \mathcal{M}_{\overline{\mathcal{F}}}^{\left(-m+\frac{1}{2}\right)}-\delta_{\overline{\mathcal{F}}}^{\left(-m+\frac{1}{2}\right)} \mathcal{M}_{\overline{\mathcal{A}}}^{(-n)}-\left[\mathcal{M}_{\overline{\mathcal{A}}}^{(-n)}, \mathcal{M}_{\overline{\mathcal{F}}}^{\left(-m+\frac{1}{2}\right)}\right]=\mathcal{M}_{[\overline{\mathcal{A}}, \overline{\mathcal{F}}]}^{\left(-n-m+\frac{1}{2}\right)} \\
\delta_{\overline{\mathcal{F}}_{1}}^{\left(-n+\frac{1}{2}\right)} \mathcal{M}_{\overline{\mathcal{F}}_{2}}^{\left(-m+\frac{1}{2}\right)}+\delta_{\overline{\mathcal{F}}_{2}}^{\left(-m+\frac{1}{2}\right)} \mathcal{M}_{\overline{\mathcal{F}}_{1}}^{\left(-n+\frac{1}{2}\right)}-\left\{\mathcal{M}_{\overline{\mathcal{F}}_{1}}^{\left(-n+\frac{1}{2}\right)}, \mathcal{M}_{\overline{\mathcal{F}}_{2}}^{\left(-m+\frac{1}{2}\right)}\right\}=\mathcal{M}_{\left\{\overline{\mathcal{F}}_{1}, \overline{\mathcal{F}}_{2}\right\}}^{(-n+1)}
\end{array}
$$

In the present case, as explained above, the supermatrices $\overline{\mathcal{A}}^{(n)}, \overline{\mathcal{A}}_{1,2}^{(n)}$ are subject to the condition $\overline{\mathcal{A}}^{(n)} \neq \mathbb{1}, \overline{\mathcal{A}}_{1,2}^{(n)} \neq \mathbb{1}$. Relations (167)-(169) imply, in complete analogy with (106), that the corresponding infinite-dimensional algebra of the "negative-grade" flows (155)-(157) :

$$
\begin{gathered}
{\left[\delta_{\overline{\mathcal{A}}_{1}}^{(-n)}, \delta_{\overline{\mathcal{A}}_{2}}^{(-m)}\right]=\delta_{\left[\overline{\mathcal{A}}_{1}, \overline{\mathcal{A}}_{2}\right]}^{(-n)}, \quad, \quad\left[\delta_{\overline{\mathcal{A}}}^{(-n)}, \delta_{\overline{\mathcal{F}}}^{\left(-m+\frac{1}{2}\right)}\right]=\delta_{[\overline{\mathcal{A}}, \overline{\mathcal{F}}]}^{\left(-n-m+\frac{1}{2}\right)}} \\
\left\{\delta_{\overline{\mathcal{F}}_{1}}^{\left(-n+\frac{1}{2}\right)}, \delta_{\overline{\mathcal{F}}_{2}}^{\left(-m+\frac{1}{2}\right)}\right\}=\delta_{\left\{\overline{\mathcal{F}}_{1}, \overline{\mathcal{F}}_{2}\right\}}^{(-n+1)}
\end{gathered}
$$

is the superloop superalgebra $\left(\widehat{G L}_{N+r, N+r}^{\prime}\right)_{-}$- the negative-grade part of $\widehat{G L}_{N+r, N+r}^{\prime}$ (cf. (107)), where the prime indicates factoring out of the unit matrix in any integer-grade subspace, i.e. $\overline{\mathcal{A}}^{(-n)} \neq \mathbb{1}$, whereas $\overline{\mathcal{F}}^{\left(-n+\frac{1}{2}\right)}$ is arbitrary.

From relations (159)-(160) and (114) $-(115)$ it is straighforward to check that in bosonic constrained $\operatorname{SKP}_{\left(R ; M_{B}, M_{F}\right)}$ supersymmetric hierarchies (33) the positive-grade $\left(\widehat{G L}_{M_{B}, M_{F}}\right)_{+}$(Eqs. (106) with (92)-(96)) and negative-grade $\left(\widehat{G L}_{N+r, N+r}^{\prime}\right)_{-}$(Eqs.(170) with (155)-(157)) superloop superalgebra additional symmetries (anti-)commute among themselves.

\section{2 "Negative"-Grade Symmetries of Fermionic $S K P_{\left(R ; M_{B}, M_{F}\right)}$ Hierarchies}

Now we turn to the construction of "negative-grade" superloop superalgebra additional symmetries for $\operatorname{SKP}_{\left(R ; M_{B}, M_{F}\right)}$ hierarchies with fermionic super-Lax operators (24) (where $R=2 r+1, M \equiv$ 
$M_{B}+M_{F}=2 N+1$ ). Employing short-hand notations (53) we introduce the infinite set of superpseudo-differential operators:

$$
\begin{aligned}
\mathcal{M}_{\overline{\mathcal{A}}}^{(-\ell / 2)} & =\sum_{I, J=1}^{2(N+r+1)} \overline{\mathcal{A}}_{I J}^{(-\ell / 2)} \sum_{s=0}^{\ell-1}(-1)^{s(\ell+|J|)} \phi_{J}^{(-(\ell-s-1) / 2)} \mathcal{D}^{-1} \psi_{I}^{(-s / 2)} \\
\mathcal{M}_{\overline{\mathcal{F}}}^{(-\ell / 2)} & =\sum_{I, J=1}^{2(N+r+1)} \overline{\mathcal{F}}_{I J}^{(-\ell / 2)} \sum_{s=0}^{\ell-1}(-1)^{s(\ell+|J|)} \phi_{J}^{(-(\ell-s-1) / 2)} \mathcal{D}^{-1} \psi_{I}^{(-s / 2)}
\end{aligned}
$$

defining the supersymmetric flows:

$$
\delta_{\overline{\mathcal{A}}}^{(-\ell / 2)} \mathcal{L}=\left[\mathcal{M}_{\overline{\mathcal{A}}}^{(-\ell / 2)}, \mathcal{L}\right] \quad, \quad \delta_{\overline{\mathcal{F}}}^{(-\ell / 2)} \mathcal{L}=\left[\mathcal{M}_{\overline{\mathcal{F}}}^{(-\ell / 2)}, \mathcal{L}\right]
$$

Here $\overline{\mathcal{A}}_{I J}^{(-\ell / 2)}$ and $\overline{\mathcal{F}}_{I J}^{(-\ell / 2)}$ are constant graded matrices belonging to $\widehat{G L}(N+r+1, N+r+1)$ (cf. (70)-(72)). Furthermore, $\overline{\mathcal{A}}^{(-n)} \neq \mathbb{1}$ in Eqs.(171), (173) since $\mathcal{M}_{\overline{\mathcal{A}}=\mathbb{1}}^{(-n)}=\mathcal{L}^{-2 n}$ (cf. Eq.(32)), so that the flow $\delta_{\overline{\mathcal{A}}=\mathbb{1}}^{(-n)}$ identically vanishes according to the first Eq.(173). Recall also, that according to (57) all super-functions entering the super-pseudo-differential operators (171)-(172) are again (adjoint) super-eigenfunctions of fermionic $\operatorname{SKP}_{\left(R ; M_{B}, M_{F}\right)}$ hierachies (24).

Consistency of $\delta_{\overline{\mathcal{A}}, \overline{\mathcal{F}}}^{(-\ell / 2)}$-flow action (173) with the constrained form of $L$ (24) implies:

$$
\delta_{\overline{\mathcal{A}}, \overline{\mathcal{F}}}^{(-\ell / 2)} \Phi_{i}=\mathcal{M}_{\overline{\mathcal{A}}, \overline{\mathcal{F}}}^{(-\ell / 2)}\left(\Phi_{i}\right) \quad, \quad \delta_{\overline{\mathcal{A}}, \overline{\mathcal{F}}}^{(-\ell / 2)} \Psi_{i}=-\left(\mathcal{M}^{(-\ell / 2)}\right)_{\overline{\mathcal{A}}, \overline{\mathcal{F}}}^{*}\left(\Psi_{i}\right)
$$

or, more generally using short-hand notations (53) :

$$
\delta_{\overline{\mathcal{A}}, \overline{\mathcal{F}}}^{(-\ell / 2)} \Phi_{i}^{(m / 2)}=\mathcal{M}_{\overline{\mathcal{A}}, \overline{\mathcal{F}}}^{(-\ell / 2)}\left(\Phi_{i}^{(m / 2)}\right) \quad, \quad \delta_{\overline{\mathcal{A}}, \overline{\mathcal{F}}}^{(-\ell / 2)} \Psi_{i}^{(m / 2)}=-\left(\mathcal{M}^{(-\ell / 2)}\right)_{\overline{\mathcal{A}}, \overline{\mathcal{F}}}^{*}\left(\Psi_{i}^{(m / 2)}\right)
$$

On the other hand, consistency of $\delta_{\overline{\mathcal{A}}, \overline{\mathcal{F}}}^{(-\ell / 2)}$-flow action (173) with the constrained form of $\mathcal{L}^{-1}$ and, more generally, of $\mathcal{L}^{-K}$ (51) yields:

$$
\begin{gathered}
\delta_{\overline{\mathcal{A}}}^{(-\ell / 2)} \phi_{I}^{(-m / 2)}=\mathcal{M}_{\overline{\mathcal{A}}}^{(-\ell / 2)}\left(\phi_{I}^{(-m / 2)}\right)-\sum_{J=1}^{2(N+r+1)} \overline{\mathcal{A}}_{I J}^{(-\ell / 2)} \phi_{J}^{(-(m+\ell) / 2)} \\
\delta_{\overline{\mathcal{A}}}^{(-\ell / 2)} \psi_{I}^{(-m / 2)}=-\left(\mathcal{M}_{\overline{\mathcal{A}}}^{(-\ell / 2)}\right)^{*}\left(\psi_{I}^{(-m)}\right)+\sum_{J=1}^{2(N+r+1)}(-1)^{\ell(|J|+m)} \overline{\mathcal{A}}_{J I}^{(-\ell / 2)} \psi_{J}^{(-(m+\ell) / 2)} \\
\delta_{\overline{\mathcal{F}}}^{(-\ell / 2)} \phi_{I}^{(-m / 2)}=\mathcal{M}_{\overline{\mathcal{F}}}^{(-\ell / 2)}\left(\phi_{I}^{(-m / 2)}\right)+(-1)^{m} \sum_{J=1}^{2(N+r+1)} \overline{\mathcal{F}}_{I J}^{(-\ell / 2)} \phi_{J}^{(-(m+\ell) / 2)} \\
\delta_{\overline{\mathcal{F}}}^{(-\ell / 2)} \psi_{I}^{(-m / 2)}=-\left(\mathcal{M}_{\overline{\mathcal{F}}}^{(-\ell / 2)}\right)^{*}\left(\psi_{I}^{(-m / 2)}\right)-\sum_{J=1}^{2(N+r+1)}(-1)^{(\ell+1)(|J|+m)} \overline{\mathcal{F}}_{J I}^{(-\ell / 2)} \psi_{J}^{(-(m+\ell) / 2)}
\end{gathered}
$$

Using (176)-(179) and repeating the steps in the derivation of 80$)-(83)$ we find:

$$
\delta_{\overline{\mathcal{A}}_{1}}^{(-\ell / 2)} \mathcal{M}_{\overline{\mathcal{A}}_{2}}^{(-m / 2)}-\delta_{\overline{\mathcal{A}}_{2}}^{(-m / 2)} \mathcal{M}_{\overline{\mathcal{A}}_{1}}^{(-\ell / 2)}-\left[\mathcal{M}_{\overline{\mathcal{A}}_{1}}^{(-\ell / 2)}, \mathcal{M}_{\overline{\mathcal{A}}_{2}}^{(-m / 2)}\right]=\mathcal{M}_{\left[\overline{\mathcal{A}}_{1}, \overline{\mathcal{A}}_{2}\right]}^{(-(\ell+m) / 2)}
$$




$$
\begin{gathered}
\delta_{\overline{\mathcal{A}}}^{(-\ell / 2)} \mathcal{M}_{\overline{\mathcal{F}}}^{(-m / 2)}-\delta_{\overline{\mathcal{F}}}^{(-m / 2)} \mathcal{M}_{\overline{\mathcal{A}}}^{(-\ell / 2)}-\left[\mathcal{M}_{\overline{\mathcal{A}}}^{(-\ell / 2)}, \mathcal{M}_{\overline{\mathcal{F}}}^{(-m / 2)}\right]= \begin{cases}\mathcal{M}_{[\overline{\mathcal{A}}, \overline{\mathcal{F}}]}^{(-\ell) / 2)} & \text { for } \ell=\text { even } \\
-\mathcal{M}_{\{\overline{\mathcal{A}}, \overline{\mathcal{F}}\}}^{(-(\ell+m) / 2)} & \text { for } \ell=\text { odd }\end{cases} \\
\delta_{\overline{\mathcal{F}}_{1}}^{(-\ell / 2)} \mathcal{M}_{\overline{\mathcal{F}}_{2}}^{(-m / 2)}+\delta_{\overline{\mathcal{F}}_{2}}^{(-m / 2)} \mathcal{M}_{\overline{\mathcal{F}}_{1}}^{(-\ell / 2)}-\left\{\mathcal{M}_{\overline{\mathcal{F}}_{1}}^{(-\ell / 2)}, \mathcal{M}_{\overline{\mathcal{F}}_{2}}^{(-m / 2)}\right\}= \\
= \begin{cases} \pm \mathcal{M}_{\left\{\overline{\mathcal{F}}_{1}, \overline{\mathcal{F}}_{2}\right\}}^{(-\ell+m) / 2)} & \text { for }(\ell, m)=(\text { odd }, \text { odd }) /(\text { even, even }) \\
\pm \mathcal{M}_{\left[\overline{\mathcal{F}}_{1}, \overline{\mathcal{F}}_{2}\right]}^{(-\ell+m) / 2)} & \text { for }(\ell, m)=(\text { odd, even }) /(\text { even }, \text { odd })\end{cases}
\end{gathered}
$$

which has the same form as $(80)-(82)$, but now $\overline{\mathcal{A}}, \overline{\mathcal{A}}_{1,2}, \overline{\mathcal{F}}, \overline{\mathcal{F}}_{1,2}$ are graded matrices of bigger size belonging to $\widehat{G L}(N+r+1, N+r+1)$. Relations 180-182 imply the following infinite-dimensional algebra of flows (cf. (83)) :

$$
\begin{aligned}
& {\left[\delta_{\overline{\mathcal{A}}_{1}}^{(-\ell / 2)}, \delta_{\overline{\mathcal{A}}_{2}}^{(-m / 2)}\right]=\delta_{\left[\overline{\mathcal{A}}_{1}, \overline{\mathcal{A}}_{2}\right]}^{(-(\ell+m) / 2)}} \\
& {\left[\delta_{\overline{\mathcal{A}}}^{(-\ell / 2)}, \delta_{\overline{\mathcal{F}}}^{(-m / 2)}\right]=\delta_{[\overline{\mathcal{A}}, \overline{\mathcal{F}}]}^{(-(\ell+m) / 2)} \quad \text { for } \ell=\text { even } \quad, \quad\left[\delta_{A}^{(-\ell / 2)}, \delta_{F}^{(-m / 2)}\right]=-\delta_{\{A, F\}}^{(-(\ell+m) / 2)} \quad \text { for } \ell=\text { odd }} \\
& \left\{\delta_{\overline{\mathcal{F}}_{1}}^{(-\ell / 2)}, \delta_{\overline{\mathcal{F}}_{2}}^{(-m / 2)}\right\}= \pm \delta_{\left\{\overline{\mathcal{F}}_{1}, \overline{\mathcal{F}}_{2}\right\}}^{(-(\ell+m) / 2)} \quad \text { for }(\ell, m)=(\text { odd }, \text { odd }) /(\text { even, even }) \\
& \left\{\delta_{\overline{\mathcal{F}}_{1}}^{(-\ell / 2)}, \delta_{\overline{\mathcal{F}}_{2}}^{(-m / 2)}\right\}= \pm \delta_{\left[\overline{\mathcal{F}}_{1}, \overline{\mathcal{F}}_{2}\right]}^{(-(\ell+m) / 2)} \quad \text { for }(\ell, m)=(\text { odd, even }) /(\text { even }, \text { odd })
\end{aligned}
$$

which is isomorphic to $\left(\widehat{G L}^{\prime}(N+r+1, N+r+1)\right)_{-}$. The latter is the negative-grade part of $\widehat{G L}(N+r+1, N+r+1)\left(\mathrm{cf} .(70)-(\sqrt[72]{ })\right.$ ), where the prime indicates factoring out $\overline{\mathcal{A}}^{(-n)}=\mathbb{1}$ in each integer-grade subspace (recall that the flows $\delta_{\overline{\mathcal{A}}=\mathbb{1}}^{(-n)}(173)$ vanish identically).

Finally, using (91) and (175), (176)-(179), we get:

$$
\begin{aligned}
& \delta_{\mathcal{A}}^{(\ell / 2)} \mathcal{M}_{\overline{\mathcal{A}}}^{(-m / 2)}-\delta_{\overline{\mathcal{A}}_{2}}^{(-m / 2)} \mathcal{M}_{\mathcal{A}}^{(\ell / 2)}-\left[\mathcal{M}_{\mathcal{A}}^{(\ell / 2)}, \mathcal{M}_{\overline{\mathcal{A}}}^{(-m / 2)}\right]=0 \\
& \delta_{\mathcal{A}}^{(\ell / 2)} \mathcal{M}_{\overline{\mathcal{F}}}^{(-m / 2)}-\delta_{\overline{\mathcal{F}}_{2}}^{(-m / 2)} \mathcal{M}_{\mathcal{A}}^{(\ell / 2)}-\left[\mathcal{M}_{\mathcal{A}}^{(\ell / 2)}, \mathcal{M}_{\overline{\mathcal{F}}}^{(-m / 2)}\right]=0 \\
& \delta_{\mathcal{F}}^{(\ell / 2)} \mathcal{M}_{\overline{\mathcal{A}}}^{(-m / 2)}-\delta_{\overline{\mathcal{A}}_{2}}^{(-m / 2)} \mathcal{M}_{\mathcal{F}}^{(\ell / 2)}-\left[\mathcal{M}_{\mathcal{F}}^{(\ell / 2)}, \mathcal{M}_{\overline{\mathcal{A}}}^{(-m / 2)}\right]=0 \\
& \delta_{\mathcal{F}}^{(\ell / 2)} \mathcal{M}_{\overline{\mathcal{F}}}^{(-m / 2)}+\delta_{\overline{\mathcal{F}}_{2}}^{(-m / 2)} \mathcal{M}_{\mathcal{F}}^{(\ell / 2)}-\left\{\mathcal{M}_{\mathcal{F}}^{(\ell / 2)}, \mathcal{M}_{\overline{\mathcal{F}}}^{(-m / 2)}\right\}=0
\end{aligned}
$$

Relations (184)-(187) imply that in fermionic constrained $\operatorname{SKP}_{\left(R ; M_{B}, M_{F}\right)}$ supersymmetric hierarchies (24) positive-grade $\left(\widehat{G L}\left(M_{B}, M_{F}\right)\right)_{+}$symmetry flows (anti-)commute with negative-grade $(\widehat{G L}(N+r+1, N+r+1))_{-}$symmetry flows (recall $\left.M \equiv M_{B}+M_{F}=2 N+1\right)$ :

$$
\left[\delta_{\mathcal{A}}^{(\ell / 2)}, \delta_{\overline{\mathcal{A}}}^{(-m / 2)}\right]=0 \quad, \quad\left[\delta_{\mathcal{A}, \mathcal{F}}^{(\ell / 2)}, \delta_{\overline{\mathcal{F}}, \overline{\mathcal{A}}}^{(-m / 2)}\right]=0 \quad, \quad\left\{\delta_{\mathcal{F}}^{(\ell / 2)}, \delta_{\overline{\mathcal{F}}}^{(-m / 2)}\right\}=0
$$

\subsection{Full Superloop Superalgebra Additional Symmetries}

Collecting the results from Sections 5,6 and the present section we conclude that:

- Fermionic constrained $\operatorname{SKP}_{\left(R ; M_{B}, M_{F}\right)}$ supersymmetric hierarchies (24) (where $R=2 r+1$, $\left.M \equiv M_{B}+M_{F}=2 N+1\right)$ possess the following superloop superalgebra symmetries:

$$
\left(\widehat{G L}\left(M_{B}, M_{F}\right)\right)_{+} \oplus\left(\widehat{G L}^{\prime}(N+r+1, N+r+1)\right)_{-}
$$


- Bosonic constrained $\operatorname{SKP}_{\left(R ; M_{B}, M_{F}\right)}$ supersymmetric hierarchies (33) (where $R=2 r, M \equiv$ $\left.M_{B}+M_{F}=2 N\right)$ possess the following superloop superalgebra symmetries:

$$
\left(\widehat{G L}_{M_{B}, M_{F}}\right)_{+} \oplus\left(\widehat{G L}_{N+r, N+r}^{\prime}\right)_{-}
$$

\section{Virasoro Symmetries of Constrained SKP Hierarchies}

The action of the operators - (multiplication by) $\lambda$ and $\eta$ as well as $\frac{\partial}{\partial \lambda}$ and $\frac{\partial}{\partial \eta}$ on the "free" BA super-function $\psi_{B A}^{(0)}$ (15)-(16) can be expressed as the action of the following superspace operators (cf. third ref. [1]) :

$$
\lambda \psi_{B A}^{(0)}=\partial \psi_{B A}^{(0)} \quad, \quad \frac{\partial}{\partial \lambda} \psi_{B A}^{(0)}=\Gamma_{0} \psi_{B A}^{(0)} \quad, \quad \eta \psi_{B A}^{(0)}=-\left(Q+\Gamma_{1} \partial\right) \psi_{B A}^{(0)} \quad, \quad \frac{\partial}{\partial \eta} \psi_{B A}^{(0)}=\Gamma_{1} \psi_{B A}^{(0)}
$$

where $Q=\frac{\partial}{\partial \theta}-\theta \partial$ is the standard super-charge operator and:

$$
\begin{gathered}
\Gamma_{0} \equiv \sum_{l=1}^{\infty} l t_{l} \partial^{l-1}+\sum_{n=1}^{\infty}\left(n-\frac{1}{2}\right) \theta_{n} \partial^{n-2} \mathcal{D}-\frac{1}{2} \sum_{n=1}^{\infty} \theta_{n} \partial^{n-2} Q+\frac{1}{2} \sum_{n, l=1}^{\infty}(n-l) \theta_{n} \theta_{l} \partial^{n+l-2} \\
\Gamma_{1} \equiv \theta+\sum_{n=1}^{\infty} \theta_{n} \partial^{n-1} \quad, \quad Q+\Gamma_{1} \partial=\frac{\partial}{\partial \theta}+\sum_{n=1}^{\infty} \theta_{n} \partial^{n}
\end{gathered}
$$

Dressing arbitrary products of powers of the above "free" superspace operators by means of Sato superspace dressing operator $\mathcal{W}(5)$ :

$$
\mathcal{M}_{k, \ell, m, n}=\left(\mathcal{W} \Gamma_{0}^{k} \partial^{\ell} \Gamma_{1}^{m}\left(Q+\Gamma_{1} \partial\right)^{n} \mathcal{W}^{-1}\right)_{-}
$$

defines via Eqs.(58) an infinite set of bosonic and fermionic symmetry flows for the general unconstrained MR-SKP hierarchy (4) which span the supersymmetric version of $W_{1+\infty}$ algebra [1]. For the class of reduced $S K P_{\left(R ; M_{B}, M_{F}\right)}$ hierarchies (24), however, the flows constructed by (194) $d o$ not define symmetries since they do not preserve the constrained form of the pertinent super-Lax operators. This is a superspace analog of the problem with the usual Orlov-Schulman operators [18], which do not yield symmetries in the case of constrained KP hierarchies in the purely bosonic case. In the present Section we will follow our approach from refs. [21] where the latter problem has been solved via appropriate modification of the standard additional-symmetry generating Orlov-Schulman operators.

In fact, we will construct here the Virasoro additional symmetries for bosonic constrained $\operatorname{SKP}_{\left(R ; M_{B}, M_{F}\right)}$ hierarchies (33). This same construction based on the super-pseudo-differential formalism does not, however, carry over to the case of the fermionic part of the full super-Virasoro and the rest of super- $W_{1+\infty}$ symmetries, as well to the case of (super-)Virasoro and super- $W_{1+\infty}$ symmetries for fermionic constrained $\operatorname{SKP}_{\left(R ; M_{B}, M_{F}\right)}$ hierarchies (24).

Similarly to the purely bosonic case [21], the action of Virasoro flows on super-Lax and superdressing operators are given by (henceforth $L \equiv \mathcal{L}_{\left(2 r ; M_{B}, M_{F}\right)}$ and we employ notations from Section 6 above) :

$$
\delta_{n}^{V} L=\left[-\left(\mathcal{W} \stackrel{(0)}{\mathcal{M}}_{n} \mathcal{W}^{-1}\right)_{-}+\mathcal{X}_{n}, L\right] \quad, \quad \delta_{n}^{V} \mathcal{W}=\left(-\left(\mathcal{W} \stackrel{(0)}{\mathcal{M}} \mathcal{W}^{-1}\right)_{-}+\mathcal{X}_{n}\right) \mathcal{W}
$$


or, equivalently:

$$
\delta_{n}^{V} L=\left[\left(\mathcal{W} \stackrel{(0)}{\mathcal{M}} \mathcal{W}^{-1}\right)_{+}+\mathcal{X}_{n}, L\right]+L^{n}
$$

where $\delta_{n}^{V} \simeq-L_{n-1}$ (in terms of standard Virasoro notations). Here:

$$
\stackrel{(0)}{\mathcal{M}} n \equiv \Gamma_{0} \partial^{n}+\frac{n}{2} \Gamma_{1}\left(Q+\Gamma_{1} \partial\right) \partial^{n-1}
$$

are the "bare" (undressed) Virasoro operators and the additional operators $\mathcal{X}_{n}$ are to be chosen in such a way that the flows (195) define a symmetry, i.e., they must preserve the constrained form of $L \equiv L_{r, N}$ (33).

For non-negative Virasoro flows $\left(n \geq 0\right.$ in (195)) we find the following expression for $\mathcal{X}_{n}$ :

$$
\mathcal{X}_{n} \equiv \sum_{s=1}^{n-1}\left(s-\frac{n}{2}\right)\left[\sum_{a=1}^{M_{B}} \Phi_{a}^{(n-s)} D^{-1} \widetilde{\Psi}_{a}^{(s)}+\sum_{b=1}^{M_{F}} \widetilde{\Phi}_{b}^{(n-s)} D^{-1} \Psi_{b}^{(s)}\right]
$$

where the short-hand notations (45) are used. Consistency of (195) with the constrained form of $L$ (33) and its inverse powers (47) dictates the specific form of the action of $\delta_{n}^{V}$-flows (for $n \geq 0$ ) on the pertinent (adjoint) super-eigenfunctions (45) and (41)-(42), which reads accordingly:

$$
\begin{aligned}
& \delta_{n}^{V} \stackrel{(\sim)}{\Phi_{a}^{(m)}}=\left[\left(\mathcal{W} \stackrel{(0)}{\mathcal{M}}_{n} \mathcal{W}^{-1}\right)_{+}+\mathcal{X}_{n}\right]\left(\stackrel{(\sim)}{\Phi}_{a}^{(m)}\right)+\left(\frac{n}{2}+m-1\right) \stackrel{\left.(\sim)^{(}\right)}{(n+m-1)} \\
& \left.\delta_{n}^{V} \stackrel{(\sim)}{\Psi}_{a}^{(m)}=-\left[\left(\mathcal{W} \stackrel{(0)}{\mathcal{M}}_{n} \mathcal{W}^{-1}\right)_{+}^{*}+\mathcal{X}_{n}^{*}\right] \stackrel{\left(\sim^{\Psi}\right.}{\Psi_{a}^{(m)}}\right)+\left(\frac{n}{2}+m-1\right) \stackrel{(\sim)}{\Psi}_{a}^{(n+m-1)} \\
& \delta_{n}^{V} \stackrel{(\sim)}{\Phi_{b}^{(-m)}}=\left[\left(\mathcal{W} \stackrel{(0)}{\mathcal{M}}{ }_{n} \mathcal{W}^{-1}\right)_{+}+\mathcal{X}_{n}\right]\left(\stackrel{(\sim)}{\Phi}_{b}^{(-m)}\right)-(m-1) \stackrel{\left.(\sim)^{(}\right)}{(-(m-n+1))} \quad \text { for } m \geq n \\
& \delta_{n}^{V} \stackrel{(\sim)}{\Phi_{b}^{(-m)}}=\left[\left(\mathcal{W} \stackrel{(0)}{\mathcal{M}}{ }_{n} \mathcal{W}^{-1}\right)_{+}+\mathcal{X}_{n}\right]\left(\stackrel{(\sim)}{\Phi}_{b}^{(-m)}\right) \text { for } m \leq n-1 \\
& \delta_{n}^{V} \stackrel{(\sim)}{\Psi}_{b}^{(-m)}=-\left[\left(\mathcal{W} \stackrel{(0)}{\mathcal{M}}_{n} \mathcal{W}^{-1}\right)_{+}^{*}+\mathcal{X}_{n}^{*}\right]\left(\stackrel{(\sim)}{\Psi}_{b}^{(-m)}\right)-(m-1) \stackrel{(\sim)}{\Psi}_{b}^{-(m-n+1))} \text { for } m \geq n \\
& \delta_{n}^{V} \stackrel{(\sim)}{\Psi}_{b}^{(-m)}=-\left[\left(\mathcal{W} \stackrel{(0)}{\mathcal{M}}_{n} \mathcal{W}^{-1}\right)_{+}^{*}+\mathcal{X}_{n}^{*}\right]\left(\stackrel{(\sim)}{\Psi}_{b}^{(-m)}\right) \text { for } m \leq n-1
\end{aligned}
$$

where relations (47) are taken into account.

For negative Virasoro flows $(n<0$ in (195)) we obtain (employing again notations (41)-(42)) :

$$
\mathcal{X}_{(-|n|)}=\sum_{b=1}^{N+r} \sum_{j=0}^{|n|}\left(\frac{|n|}{2}-j\right)\left[\Phi_{b}^{-(|n|-j+1)} \mathcal{D}^{-1} \widetilde{\Psi}_{b}^{-(j+1)}+\widetilde{\Phi}_{b}^{-(|n|-j+1)} \mathcal{D}^{-1} \Psi_{b}^{-(j+1)}\right]
$$

Consistency of (195) (for $n<0$ ) with the constrained form of $L(33)$ and its inverse powers (43) implies that the flows $\delta_{-|n|}^{V}$ act on the constituent (adjoint) super-eigenfunctions (45) and (41)-(42) as follows (taking into account (47)) :

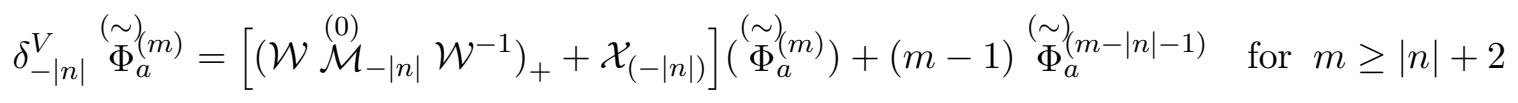

$$
\begin{aligned}
& \delta_{-|n|}^{V} \stackrel{(\sim)}{\Phi_{a}^{(m)}}=\left[\left(\mathcal{W} \stackrel{(0)}{\mathcal{M}}_{-|n|} \mathcal{W}^{-1}\right)_{+}+\mathcal{X}_{(-|n|)}\right] \stackrel{\left(\stackrel{(\sim)}{\Phi}_{a}^{(m)}\right)}{\text { for }} m \leq|n|+1
\end{aligned}
$$




$$
\begin{aligned}
& \delta_{-|n|}^{V} \stackrel{(\sim)}{\Psi}_{a}^{(m)}=-\left[\left(\mathcal{W} \stackrel{(0)}{\mathcal{M}}_{-|n|} \mathcal{W}^{-1}\right)_{+}^{*}+\mathcal{X}_{(-|n|)}^{*}\right]\left(\stackrel{(\sim)}{\Phi}_{a}^{(m)}\right)+(m-1) \stackrel{(\sim)}{\Psi}_{a}^{(m-|n|-1)} \quad \text { for } m \geq|n|+2 \\
& \delta_{-|n|}^{V} \stackrel{(\sim)}{\Psi}_{a}^{(m)}=-\left[\left(\mathcal{W} \stackrel{(0)}{\mathcal{M}}_{-|n|} \mathcal{W}^{-1}\right)_{+}^{*}+\mathcal{X}_{(-|n|)}^{*}\right]\left(\stackrel{(\sim)}{\Phi}_{a}^{(m)}\right) \quad \text { for } m \leq|n|+1
\end{aligned}
$$

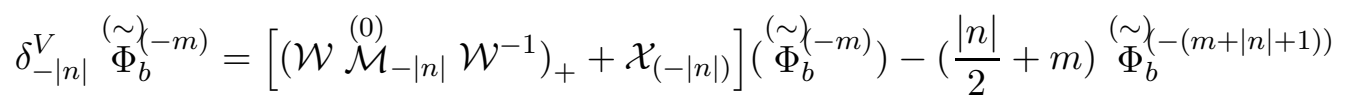

$$
\begin{aligned}
& \delta_{-|n|}^{V} \stackrel{(\sim \sim}{\Psi}_{b}^{(-m)}=-\left[\left(\mathcal{W} \stackrel{(0)}{\mathcal{M}}_{-|n|} \mathcal{W}^{-1}\right)_{+}^{*}+\mathcal{X}_{(-|n|)}^{*}\right]\left(\stackrel{(\sim)}{\Psi}_{b}^{(-m)}\right)-\left(\frac{|n|}{2}+m\right) \stackrel{(\sim)}{\Psi}_{b}^{(-(m+|n|+1))}
\end{aligned}
$$

The consistency of the negative flow definitions (195) (or (196)) with $n<0$, where $\mathcal{X}_{(-|n|)}$ is as in Eq. 205), crucially depends on relations (47). Also, in the process of derivation of (198)-(211) essential use is made of the super-pseudo-differential operator identities (31).

What is left is to check that the flows (195) indeed satisfy the commutation relations of the standard Virasoro algebra. To this end let us consider the commutator of the Virasoro flows $\delta_{n}^{V} \simeq-L_{n-1}$ and $\delta_{m}^{V} \simeq-L_{m-1}$ (with $L_{n}$ being the standard notations for the basis of Virasoro algebra) acting on the bosonic super-Lax operator $L$ (where $(n, m)$ are arbitrary non-negative or negative indices) which yields:

$$
\begin{array}{r}
\delta_{n}^{V}\left(-\left(\mathcal{W} \stackrel{(0)}{\mathcal{M}} \mathcal{W}_{m}^{-1}\right)_{-}+\mathcal{X}_{m}\right)-\delta_{m}^{V}\left(-\left(\mathcal{W} \stackrel{(0)}{\mathcal{M}}_{n} \mathcal{W}^{-1}\right)_{-}+\mathcal{X}_{n}\right) \\
-\left[-\left(\mathcal{W} \stackrel{(0)}{\mathcal{M}}_{n} \mathcal{W}^{-1}\right)_{-}+\mathcal{X}_{n},-\left(\mathcal{W} \stackrel{(0)}{\mathcal{M}}_{m} \mathcal{W}^{-1}\right)_{-}+\mathcal{X}_{m}\right]
\end{array}
$$

Using the identity:

$$
\begin{array}{r}
\delta_{n}^{V}\left(\mathcal{W} \stackrel{(0)}{\mathcal{M}}_{m} \mathcal{W}^{-1}\right)_{-}-\delta_{m}^{V}\left(\mathcal{W} \stackrel{(0)}{\mathcal{M}}_{n} \mathcal{W}^{-1}\right)_{-}=-(n-m)\left(\mathcal{W} \stackrel{(0)}{\mathcal{M}}_{n+m-1} \mathcal{W}^{-1}\right)_{-} \\
-\left[\left(\mathcal{W} \stackrel{(0)}{\mathcal{M}}_{n} \mathcal{W}^{-1}\right)_{-},\left(\mathcal{W} \stackrel{(0)}{\mathcal{M}}_{m} \mathcal{W}^{-1}\right)_{-}\right]+\left[\mathcal{X}_{n}, \mathcal{W} \stackrel{(0)}{\mathcal{M}}_{m} \mathcal{W}^{-1}\right]_{-}-\left[\mathcal{X}_{m}, \mathcal{W} \stackrel{(0)}{\mathcal{M}}_{n} \mathcal{W}^{-1}\right]_{-}
\end{array}
$$

the r.h.s. of Eq.(212) can be rewritten in the form:

$$
\begin{array}{r}
(n-m)\left(\mathcal{W} \stackrel{(0)}{\mathcal{M}}{ }_{n+m-1} \mathcal{W}^{-1}\right)_{-}+\delta_{n}^{V} \mathcal{X}_{m}-\left[\left(\mathcal{W} \stackrel{(0)}{\mathcal{M}}_{n} \mathcal{W}^{-1}\right)_{+}, \mathcal{X}_{m}\right]_{-} \\
-\delta_{m}^{V} \mathcal{X}_{n}+\left[\left(\mathcal{W} \stackrel{(0)}{\mathcal{M}} \mathcal{W}^{-1}\right)_{+}, \mathcal{X}_{n}\right]_{-}-\left[\mathcal{X}_{n}, \mathcal{X}_{m}\right]
\end{array}
$$

Now, employing again the super-pseudo-differential identities (31) we find, taking into account (199)-(204) and (206)-(211), that the sum of all terms in (214) involving $\mathcal{X}_{n, m}$ yield:

$\delta_{n}^{V} \mathcal{X}_{m}-\left[\left(\mathcal{W} \stackrel{(0)}{\mathcal{M}} \mathcal{W}^{-1}\right)_{+}, \mathcal{X}_{m}\right]_{-}-\delta_{m}^{V} \mathcal{X}_{n}+\left[\left(\mathcal{W} \stackrel{(0)}{\mathcal{M}} \mathcal{W}^{-1}\right)_{+}, \mathcal{X}_{n}\right]_{-}-\left[\mathcal{X}_{n}, \mathcal{X}_{m}\right]=-(n-m) \mathcal{X}_{n+m-1}$

Thus, we verify the closure of the full Virasoro algebra of additional symmetries without central extension:

$$
\left[\delta_{n}^{V}, \delta_{m}^{V}\right]=-(n-m) \delta_{n+m-1}^{V}
$$

\section{Superspace Darboux-Bäcklund Transformations and Wronskian-like Super-Determinant Solutions}

10.1 Darboux-Bäcklund Transformations for Constrained Super-KP Hierarchies 
In what follows we shall consider Darboux-Bäcklund (DB) transformations for the whole class $\operatorname{SKP}_{\left(R ; M_{B}, M_{F}\right)}$ of constrained (reduced) supersymmetric KP integrable hierarchies (24). For definiteness we shall explicitly discuss the case of $S K P_{\left(R ; M_{B}, M_{F}\right)}$ hierarchies defined by fermionic super-Lax operators. DB and adjoint-DB transformed objects will be indicated by tilde and hat, respectively, on top of the corresponding symbol.

In analogy with the ordinary "bosonic" case, DB transformations within the Sato super-pseudodifferential operator approach are defined as "gauge" transformations of special kind on the pertinent super-Lax operator of the supersymmetric integrable hierarchy:

$$
\mathcal{L} \quad \rightarrow \quad \widetilde{\mathcal{L}}=\mathcal{T}_{\phi} \mathcal{L}_{\phi}^{-1} \quad, \quad \mathcal{T}_{\phi} \equiv \phi \mathcal{D} \phi^{-1}
$$

with $\phi$ being a bosonic superfunction, which obey the following requirements:

(A) Super-DB transformations (217) have to preserve the specific constrained form (24) of $\mathcal{L}$ (or (33) for bosonic $\operatorname{SKP}_{\left(R ; M_{B}, M_{F}\right)}$ hierarchies), i.e., the transformed super-Lax operator $\widetilde{\mathcal{L}}$ (217) must be again of the form:

$$
\widetilde{\mathcal{L}} \equiv \widetilde{\mathcal{L}}_{\left(R ; \widetilde{M}_{B}, \widetilde{M}_{F}\right)}=\mathcal{D}^{R}+\sum_{j=0}^{R-1} \widetilde{v}_{\frac{j}{2}} \mathcal{D}^{j}+\sum_{i=1}^{M} \widetilde{\Phi}_{i} \mathcal{D}^{-1} \widetilde{\Psi}_{i} \quad, \quad M=\widetilde{M}_{B}+\widetilde{M}_{F}
$$

where $\widetilde{M}_{B, F}$ are the numbers of DB-transformed bosonic/fermionic (adjoint) super-eigenfunctions $\widetilde{\Phi}_{i}, \widetilde{\Psi}_{i}$. Let us stress that we require the total number $M$ of negative super-pseudo-differential terms in $\widetilde{\mathcal{L}}$ to be the same as in the initial super-Lax operator $\mathcal{L}(24)$. Let us also note that, using the superspace pseudo-differential operator identities (31), the DB-transformed fermionic $\operatorname{SKP}_{\left(R ; M_{B}, M_{F}\right)}$ super-Lax operator 217) acquires the form:

$$
\widetilde{\mathcal{L}}=(\widetilde{\mathcal{L}})_{+}+\left(\mathcal{T}_{\phi} \mathcal{L}(\phi)\right) \mathcal{D}^{-1} \phi^{-1}+\sum_{i=1}^{M}(-1)^{|i|} \mathcal{T}_{\phi}\left(\Phi_{i}\right) \mathcal{D}^{-1}\left(\mathcal{T}_{\phi}^{-1}\right)^{*}\left(\Psi_{i}\right)
$$

Therefore, one of the $M+1$ negative super-pseudo-differential terms on the r.h.s. of (219) has to vanish.

(B) Super-DB transformations (217) have to preserve the bosonic (30) and fermionic (25) isospectral evolution equations (in the case of fermionic $S K P_{\left(R ; M_{B}, M_{F}\right)}$ hierarchies) or Eqs.(34) (for bosonic $\operatorname{SKP}_{\left(R ; M_{B}, M_{F}\right)}$ hierarchies). As we will see below, the fermionic isospectral flows (25) can be strictly preserved under super-DB transformations only for the subclass $S K P_{(R ; 1,0)}$ of constrained super-KP hierarchies $S K P\left(R ; M_{B}, M_{F}\right)$. In the more general case we will require preservation of fermionic isospectral flows under super-DB transformations up to an overall sign change.

Similarly, we can define adjoint-DB transformations:

$$
\mathcal{L} \rightarrow \widehat{\mathcal{L}}=\left(-\mathcal{T}_{\psi}^{-1}\right)^{*} \mathcal{L} \mathcal{T}_{\psi}^{*} \quad, \quad \mathcal{T}_{\psi} \equiv \psi \mathcal{D} \psi^{-1}
$$

obeying the same requirements (A) and (B). In this case the counterpart of Eq.(219) now reads:

$$
\widehat{\mathcal{L}}=(\widehat{\mathcal{L}})_{+}+\psi^{-1} \mathcal{D}^{-1}\left(\mathcal{T}_{\psi} \mathcal{L}^{*}(\psi)\right)+\sum_{i=1}^{M}(-1)^{|i|}\left(-\mathcal{T}_{\psi}^{-1}\right)^{*}\left(\Phi_{i}\right) \mathcal{D}^{-1} \mathcal{T}_{\psi}\left(\Psi_{i}\right)
$$

As in (219), one of the $M+1$ negative super-pseudo-differential terms on the r.h.s. of (221) has to vanish. 
Comparing (219) with (218) (and similarly for the adjoint-DB transformations (221)), and taking into account relations (55) (for fermionic super-Lax operators) or (46) (for bosonic superLax operators), we find that condition (A) above can be satisfied for two different choices of the (adjoint-)DB generating superfunctions $\phi$ and $\psi$ :

(i) First choice: $\phi=\Phi_{i_{0}}$ where $\Phi_{i_{0}}$ is some fixed bosonic super-eigenfunction entering the negative pseudo-differential part of the original super-Lax operator (24). In this case we obtain:

$$
\begin{gathered}
\widetilde{\Phi}_{i_{0}}=\mathcal{T}_{\phi} \mathcal{L}(\phi) \quad, \quad \widetilde{\Psi}_{i_{0}}=\phi^{-1} \quad, \quad \phi \equiv \Phi_{i_{0}} \\
\widetilde{\Phi}_{i}=\mathcal{T}_{\phi}\left(\Phi_{i}\right) \quad, \quad \widetilde{\Psi}_{i}=(-1)^{|i|}\left(\mathcal{T}_{\phi}^{-1}\right)^{*}\left(\Psi_{i}\right) \quad, \quad i \neq i_{0}
\end{gathered}
$$

Similarly, the first choice for adjoint-DB transformations is $\psi=\Psi_{i_{0}}$ where $\Psi_{i_{0}}$ is some fixed bosonic adjoint super-eigenfunction entering the negative pseudo-differential part of the original super-Lax operator (24). Accordingly, for the adjoint-DB transformed (adjoint) super-eigenfunctions we have:

$$
\begin{gathered}
\widehat{\Phi}_{i_{0}}=-\psi^{-1}, \quad \widehat{\Psi}_{i_{0}}=-\mathcal{T}_{\psi} \mathcal{L}^{*}(\psi) \quad, \quad \psi \equiv \Psi_{i_{0}} \\
\widehat{\Phi}_{i}=(-1)^{|i|}\left(\mathcal{T}_{\psi}^{-1}\right)^{*}\left(\Phi_{i}\right) \quad, \quad \widehat{\Psi}_{i}=-\mathcal{T}_{\psi}\left(\Psi_{i}\right) \quad, \quad i \neq i_{0}
\end{gathered}
$$

Let us note that the Grassmann parity of the DB transformed (adjoint) super-eigenfunctions $\widetilde{\Phi}_{i}$ and $\widetilde{\Psi}_{i}(223)$ for $i \neq i_{0}$ changes from $|i|$ to $|i|+1$, and similarly for the adjoint-DB transformed ones (225).

(ii) Second choice: $\phi=L_{N+\frac{1}{2}}\left(\widetilde{\varphi}_{\alpha_{0}}\right)$ (for super-DB transformations) and $\psi=\psi_{\alpha_{0}}$ (for adjoint super-DB transformations) where $L_{N+\frac{1}{2}}\left(\widetilde{\varphi}_{\alpha_{0}}\right)$ and $\psi_{\alpha_{0}}$ are some fixed bosonic (adjoint) supereigenfunctions (52)-(53) entering the expression (50) for the inverse power of the super-Lax operator $\mathcal{L}$. Since according to (55) the defined above $\phi$ and $\psi$ obey the relations $\mathcal{L}(\phi)=0$ and $\mathcal{L}^{*}(\psi)=0$, we get for the (adjoint) DB-transformed (adjoint) super-eigenfunctions in (219) and (221) :

$$
\begin{gathered}
\widetilde{\Phi}_{i}=\mathcal{T}_{\phi}\left(\Phi_{i}\right) \quad, \quad \widetilde{\Psi}_{i}=(-1)^{|i|}\left(\mathcal{T}_{\phi}^{-1}\right)^{*}\left(\Psi_{i}\right) \quad, \quad \phi \equiv L_{N+\frac{1}{2}}\left(\widetilde{\varphi}_{\alpha_{0}}\right) \\
\widehat{\Phi}_{i}=(-1)^{|i|}\left(\mathcal{T}_{\psi}^{-1}\right)^{*}\left(\Phi_{i}\right) \quad, \quad \widehat{\Psi}_{i}=-\mathcal{T}_{\psi}\left(\Psi_{i}\right) \quad, \quad \psi=\psi_{\alpha_{0}}
\end{gathered}
$$

for all $i=1, \ldots, M$.

For later use let us write down the (adjoint) super-DB transfomations for the whole series of (adjoint) super-eigenfunctions (54)-(53) entering in the definition of additional non-isospectral symmetry flow generating operators $(66)-(67),(75)$ and (171)-(173) :

- For the first choice (i) of DB-generating (adjoint) eigenfunctions (cf. 222)-(225)) we have:

$$
\begin{gathered}
\widetilde{\Phi}_{i_{0}}^{(\ell / 2)}=\mathcal{T}_{\phi}\left(\Phi_{i_{0}}^{((\ell+1) / 2)}\right) \quad, \quad \widetilde{\Psi}_{i_{0}}^{(\ell / 2)}=(-1)^{\ell-1}\left(\mathcal{T}_{\phi}^{-1}\right)^{*}\left(\Psi_{i_{0}}^{((\ell-1) / 2)}\right) \quad \text { for } n \geq 2 \\
\widetilde{\Psi}_{i_{0}}=\frac{1}{\phi} \equiv \frac{1}{\Phi_{i_{0}}} \\
\widetilde{\Phi}_{i}^{(\ell / 2)}=\mathcal{T}_{\phi}\left(\Phi_{i}^{(\ell / 2)}\right) \quad, \quad \widetilde{\Psi}_{i}^{(\ell / 2)}=(-1)^{|i|}\left(\mathcal{T}_{\phi}^{-1}\right)^{*}\left(\Psi_{i}^{(\ell / 2)}\right) \quad \text { for } i \neq i_{0} \\
\widetilde{\phi}_{I}^{(-\ell / 2)}=\mathcal{T}_{\phi}\left(\phi_{I}^{(-\ell / 2)}\right) \quad, \quad \widetilde{\psi}_{I}^{(-\ell / 2)}=\left(\mathcal{T}_{\phi}^{-1}\right)^{*}\left(\psi_{I}^{(-\ell / 2)}\right)
\end{gathered}
$$




$$
\begin{gathered}
\widehat{\Phi}_{i_{0}}=-\frac{1}{\psi} \equiv-\frac{1}{\Psi_{i_{0}}} \quad, \quad \widehat{\Phi}_{i_{0}}^{(\ell / 2)}=-\left(\mathcal{T}_{\psi}^{-1}\right)^{*}\left(\Phi_{i_{0}}^{((\ell-1) / 2)}\right) \quad \text { for } \ell \geq 1 \\
\widehat{\Psi}_{i_{0}}^{(\ell)}=-\mathcal{T}_{\psi}\left(\Psi_{i_{0}}^{((\ell+1) / 2)}\right) \\
\widehat{\Phi}_{i}^{(\ell / 2)}=-\left(\mathcal{T}_{\psi}^{-1}\right)^{*}\left(\Phi_{i}^{(\ell / 2)}\right) \quad, \quad \widehat{\Psi}_{i}^{(\ell / 2)}=-\mathcal{T}_{\psi}\left(\Psi_{i}^{(\ell / 2)}\right) \quad, \quad i \neq i_{0} \\
\widehat{\phi}_{I}^{(-\ell / 2)}=-\left(\mathcal{T}_{\psi}^{-1}\right)^{*}\left(\phi_{I}^{(-\ell / 2)}\right) \quad, \quad \widehat{\psi}_{I}^{(-\ell / 2)}=-\mathcal{T}_{\psi}\left(\psi_{I}^{(-\ell / 2)}\right)
\end{gathered}
$$

- For the second choice (ii) of DB-generating (adjoint) eigenfunctions (cf. (226)-(227)) we obtain:

$$
\begin{gathered}
\widetilde{\Phi}_{i}^{(\ell / 2)}=\mathcal{T}_{\phi}\left(\Phi_{i}^{(\ell / 2)}\right), \quad \widetilde{\Psi}_{i}^{(\ell / 2)}=(-1)^{|i|}\left(\mathcal{T}_{\phi}^{-1}\right)^{*}\left(\Psi_{i}^{(\ell / 2)}\right), \quad \phi \equiv L_{N+\frac{1}{2}}\left(\widetilde{\varphi}_{\alpha_{0}}\right) \equiv \phi_{\alpha_{0}}^{(0)} \\
\widetilde{\phi}_{\alpha_{0}}^{(-\ell / 2)}=\mathcal{T}_{\phi}\left(\phi_{\alpha_{0}}^{(-(\ell+1) / 2)}\right), \widetilde{\psi}_{\alpha_{0}}^{(-\ell / 2)}=\left(\mathcal{T}_{\phi}^{-1}\right)^{*}\left(\psi_{\alpha_{0}}^{(-(\ell-1) / 2)}\right) \text { for } \ell \geq 1, \quad \widetilde{\psi}_{\alpha_{0}}^{(0)}=\frac{1}{\phi} \equiv \frac{1}{\phi_{\alpha_{0}}^{(0)}} \\
\widetilde{\phi}_{I}^{(-\ell / 2)}=\mathcal{T}_{\phi}\left(\phi_{I}^{(-\ell / 2)}\right), \quad \widetilde{\psi}_{I}^{(-\ell / 2)}=(-1)^{|I|}\left(\mathcal{T}_{\phi}^{-1}\right)^{*}\left(\psi_{I}^{(-\ell / 2)}\right) \quad \text { for } I \neq \alpha_{0} \\
\widehat{\Phi}_{i}^{(\ell / 2)}=(-1)^{|i|}\left(\mathcal{T}_{\psi}^{-1}\right)^{*}\left(\Phi_{i}^{(\ell / 2)}\right), \quad \widehat{\Psi}_{i}^{(\ell / 2)}=-\mathcal{T}_{\psi}\left(\Psi_{i}^{(\ell / 2)}\right), \quad \psi \equiv \psi_{\alpha_{0}} \equiv \psi_{\alpha_{0}}^{(0)} \\
\widehat{\psi}_{\alpha_{0}}^{(0)}=-\frac{1}{\psi} \equiv-\frac{1}{\psi_{\alpha_{0}}^{(0)}}, \widehat{\phi}_{\alpha_{0}}^{(-\ell / 2)}=-\left(\mathcal{T}_{\psi}^{-1}\right)^{*}\left(\phi_{\alpha_{0}}^{(-(\ell-1) / 2}\right) \text { for } \ell \geq 1 \\
\widehat{\psi}_{\alpha_{0}}^{(-\ell / 2)}=-\mathcal{T}_{\psi}\left(\Psi_{\alpha_{0}}^{(-(\ell+1) / 2)}\right) \\
\widehat{\phi}_{I}^{(-\ell / 2)}=(-1)^{|I|}\left(\mathcal{T}_{\psi}^{-1}\right)^{*}\left(\Phi_{a}^{(-\ell / 2)}\right) \quad, \quad \widehat{\psi}_{I}^{(-\ell / 2)}=-\mathcal{T}_{\psi}\left(\psi_{I}^{(-\ell / 2)}\right) \quad \text { for } I \neq \alpha_{0}
\end{gathered}
$$

Let us now study the fulfillment of condition (B) above by the (adjoint) DB transformations (222)-(223) and (224)-(225). It is straightforward to check, using the super-pseudo-differential identities (31), that the latter preserve the bosonic isospectral flow Eqs.(30) :

$$
\frac{\partial}{\partial t_{l}} \widetilde{\mathcal{L}}=\left[\frac{\partial}{\partial t_{l}} \mathcal{T}_{\phi} \mathcal{T}_{\phi}^{-1}+\mathcal{T}_{\phi}\left(\mathcal{L}^{\frac{2 l}{R}}\right)_{+} \mathcal{T}_{\phi}^{-1}, \widetilde{\mathcal{L}}\right]=\left[\left(\widetilde{\mathcal{L}}^{\frac{2 l}{R}}\right)_{+}, \widetilde{\mathcal{L}}\right]
$$

for fermionic super-Lax operators, and similarly for bosonic super-Lax operators (first Eqs.(34)). Next, we compute the action of the fermionic isospectral flows $D_{n}$ on the DB-transformed bosonic super-Lax operator $\widetilde{L}=\mathcal{T}_{\phi} L \mathcal{T}_{\phi}^{-1}$ (217) taking into account the second Eq. (34) and using the identities (31) to obtain:

$$
D_{n} \widetilde{L}=\left[D_{n} \mathcal{T}_{\phi} \mathcal{T}_{\phi}^{-1}-\mathcal{T}_{\phi}\left(L^{\frac{2 n-1}{2 r}}\right)_{+} \mathcal{T}_{\phi}^{-1}, \widetilde{L}\right]=-\left[\left(\widetilde{L}^{\frac{2 n-1}{2 r}}\right)_{+}, \widetilde{L}\right]
$$

Comparing (241) with second Eqs.(34) we note that $D_{n}$ fermionic flows for bosonic $\operatorname{SKP}\left(R ; M_{B}, M_{F}\right)$ hierarchies are preserved under (adjoit-) DB transformations up to an overall sign.

Let us now discuss the case of fermionic $\operatorname{SKP}_{\left(R ; M_{B}, M_{F}\right)}$ hierarchies. The action of the modified fermionic isospectral flows $D_{n}$ (25) on the DB-transformed fermionic super-Lax operators (217) reads:

$$
D_{n} \widetilde{\mathcal{L}}=\left\{D_{n} \mathcal{T}_{\phi} \mathcal{T}_{\phi}^{-1}+\mathcal{T}_{\phi}\left(\mathcal{L}_{-}^{2 n-1}-X_{2 n-1}\right) \mathcal{T}_{\phi}^{-1} \widetilde{\mathcal{L}}\right\}
$$


where, using the identities (31), we have:

$$
\begin{array}{r}
D_{n} \mathcal{T}_{\phi} \mathcal{T}_{\phi}^{-1}+\mathcal{T}_{\phi}\left(\mathcal{L}_{-}^{2 n-1}-X_{2 n-1}\right) \mathcal{T}_{\phi}^{-1}=\mathcal{T}_{\phi}\left(D_{n} \phi+\left(\mathcal{L}_{-}^{2 n-1}-X_{2 n-1}\right)(\phi)\right) \mathcal{D}^{-1} \phi^{-1}+ \\
+\sum_{i=1}^{M} \sum_{s=0}^{2 n-2}(-1)^{s(|i|+2 n-1)} \mathcal{T}_{\phi}\left(\mathcal{L}^{2 n-2-s}\left(\Phi_{i}\right)\right) \mathcal{D}^{-1}(-1)^{|i|+s}\left(\mathcal{T}_{\phi}^{-1}\right)^{*}\left(\left(\mathcal{L}^{s}\right)^{*}\left(\Psi_{i}\right)\right)
\end{array}
$$

For the second choice (234) of super-DB transformations we find, using (74) and (91), that the r.h.s. of Eq. (243) becomes equal to $\widetilde{\mathcal{L}}_{-}^{2 n-1}-\widetilde{X}_{2 n-1}$ where $\widetilde{X}_{2 n-1}$ is of the same form as $X_{2 n-1}$ (26) with all (adjoint) super-eigenfunctions replaced by their DB-transformed counterparts. Thus, comparing with (25) we conclude that under the second type (234) of DB transformations on fermionic $\operatorname{SKP}_{\left(R ; M_{B}, M_{F}\right)}$ super-Lax operators the fermionic isospectral flows are preserved up to an overall minus sign:

$$
D_{n} \widetilde{\mathcal{L}}=+\left\{\widetilde{\mathcal{L}}_{-}^{2 n-1}-\widetilde{X}_{2 n-1}, \widetilde{\mathcal{L}}\right\}
$$

The situation with the first type of DB transformations (228)-(229) on fermionic $\operatorname{SKP}_{\left(R ; M_{B}, M_{F}\right)}$ hierarchies is slightly more complicated. First, let us consider the subclass of $S_{K P}(R ; 1,0)$ hierarchies defined by fermionic super-Lax operators $(R=2 r+1)$ :

$$
\mathcal{L} \equiv \mathcal{L}_{(R ; 1,0)}=\mathcal{D}^{R}+\sum_{j=0}^{R-1} v_{\frac{j}{2}} \mathcal{D}^{j}+\Phi \mathcal{D}^{-1} \Psi
$$

As already shown in ref. [14, the r.h.s. of Eq.(243) becomes in this case:

$$
-\sum_{s=0}^{2 n-2}(-1)^{s} \widetilde{\mathcal{L}}^{2 n-2-s}(\widetilde{\Phi}) \mathcal{D}^{-1}\left(\widetilde{\mathcal{L}}^{s}\right)^{*}(\widetilde{\Psi})=-\left(\left(\widetilde{\mathcal{L}}^{2 n-1}\right)_{-}-\widetilde{X}_{2 n-1}\right)
$$

where we have used $\phi \equiv \Phi, \widetilde{\Phi}=\mathcal{T}_{\Phi}(\mathcal{L}(\Phi)), \widetilde{\Psi}=\Phi^{-1}$ (cf. (222) ) and also the identities from [14] :

$$
\widetilde{\mathcal{L}}^{s}(\widetilde{\Phi})=\mathcal{T}_{\Phi}\left(\mathcal{L}^{s+1}(\Phi)\right) \quad, \quad\left(\widetilde{\mathcal{L}}^{s+1}\right)^{*}(\widetilde{\Psi})=(-1)^{s}\left(\mathcal{T}_{\Phi}^{-1}\right)^{*}\left(\left(\mathcal{L}^{s}\right)^{*}(\Psi)\right)
$$

Therefore, substituting the first term in the anti-commutator in (242) with the expression (246) we conclude that for fermionic $S K P_{(R ; 1,0)}$ hierarchies (245) the fermionic isospectral flows $D_{n}(25)$ are strictly preserved (no overall sign change) under first type of super-DB transformations (222).

In the more general case of fermionic $\operatorname{SKP}_{\left(R ; M_{B}, M_{F}\right)}$ hierarchies with $M=M_{B}+M_{F} \geq 2$ the r.h.s. of Eq. 243) becomes under the first type of DB transformations (222) :

$$
-\sum_{s=0}^{2 n-2}(-1)^{s} \widetilde{\mathcal{L}}^{2 n-2-s}\left(\widetilde{\Phi}_{i_{0}}\right) \mathcal{D}^{-1}\left(\widetilde{\mathcal{L}}^{s}\right)^{*}\left(\widetilde{\Psi}_{i_{0}}\right)+\sum_{i=1, i \neq i_{0}}^{M} \sum_{s=0}^{2 n-2}(-1)^{s|i|} \widetilde{\mathcal{L}}^{2 n-2-s}\left(\widetilde{\Phi}_{i}\right) \mathcal{D}^{-1}\left(\widetilde{\mathcal{L}}^{s}\right)^{*}\left(\widetilde{\Psi}_{i}\right)
$$

which is not equal to $\pm\left(\left(\widetilde{\mathcal{L}}^{2 n-1}\right)_{-}-\widetilde{X}_{2 n-1}\right)$ due to the opposite signs in front of both sums in (248). Therefore, fermionic isospectral flows $D_{n}$ are preserved under first type of super-DB transformations only for the subclass $\operatorname{SKP}_{(R ; 1,0)}(245)$ of fermionic constrained super-KP hierarchies.

Finally, let us recall that according to [14] the super-tau function (19) undergoes the following (adjoint-)DB transformations:

$$
\tau \longrightarrow \widetilde{\tau}=\frac{\phi}{\tau} \quad, \quad \tau \longrightarrow \widehat{\tau}=-\frac{1}{\psi \tau}
$$


The latter relations are to be constrasted with their counterparts in the ordinary "bosonic" case where $\widetilde{\tau}=\phi \tau, \widehat{\tau}=-\psi \tau$.

\subsection{Superspace Darboux-Bäcklund Transformations Preserving Additional Symmetries}

We are now interested in consistency of super-DB transformations of $\operatorname{SKP}_{\left(R ; M_{B}, M_{F}\right)}$ constrained super-KP hierarchies with the whole algebra of the additional non-isospectral symmetries (Sections 5,6 and 8 above). Acting with the pertinent additional symmetry flows of positive grades ((75), (66) $-($ 677) $)$ and of negative grades $((171)-(173))$ on the DB-transformed super-Lax operator (217) (we take fermionic super-Lax operator for definiteness) we have:

$$
\delta_{\mathcal{A}, \overline{\mathcal{A}}}^{( \pm \ell / 2)} \widetilde{\mathcal{L}}=\left[\widetilde{\mathcal{M}}_{\mathcal{A}, \overline{\mathcal{A}}}^{( \pm \ell / 2)}, \widetilde{\mathcal{L}}\right] \quad, \quad \delta_{\mathcal{F}, \overline{\mathcal{F}}}^{( \pm \ell / 2)} \widetilde{\mathcal{L}}=\left\{\widetilde{\mathcal{M}}_{\mathcal{F}, \overline{\mathcal{F}}}^{( \pm \ell / 2)}, \widetilde{\mathcal{L}}\right\}
$$

Here:

$$
\begin{aligned}
& \widetilde{\mathcal{M}}_{\mathcal{A}}^{(\ell / 2)} \equiv \delta_{\mathcal{A}}^{(\ell / 2)} \mathcal{T}_{\phi} \mathcal{T}_{\phi}^{-1}+\mathcal{T}_{\phi} \mathcal{M}_{\mathcal{A}}^{(\ell / 2)} \mathcal{T}_{\phi}^{-1}=\mathcal{T}_{\phi}\left(\mathcal{M}_{\mathcal{A}}^{(\ell / 2)}(\phi)-\delta_{\mathcal{A}}^{(\ell / 2)} \phi\right) \mathcal{D}^{-1} \phi^{-1}+ \\
& +\sum_{i, j=1}^{M} \mathcal{A}_{i j}^{(\ell / 2)} \sum_{s=0}^{\ell-1}(-1)^{s(|j|+\ell)} \mathcal{T}_{\phi}\left(\Phi_{j}^{(\ell-1-s) / 2)}\right) \mathcal{D}^{-1}(-1)^{|i|+s}\left(\mathcal{T}_{\phi}^{-1}\right)^{*}\left(\Psi_{i}^{(s / 2)}\right) \\
& \widetilde{\mathcal{M}}_{\mathcal{F}}^{(\ell / 2)} \equiv \delta_{\mathcal{F}}^{(\ell / 2)} \mathcal{T}_{\phi} \mathcal{T}_{\phi}^{-1}-\mathcal{T}_{\phi} \mathcal{M}_{\mathcal{F}}^{(\ell / 2)} \mathcal{T}_{\phi}^{-1}=\mathcal{T}_{\phi}\left(\delta_{\mathcal{F}}^{(\ell / 2)} \phi-\mathcal{M}_{\mathcal{F}}^{(\ell / 2)}(\phi)\right) \mathcal{D}^{-1} \phi^{-1}+ \\
& +\sum_{i, j=1}^{M} \mathcal{F}_{i j}^{(\ell / 2)} \sum_{s=0}^{\ell-1}(-1)^{s(|j|+\ell)} \mathcal{T}_{\phi}\left(\Phi_{j}^{(\ell-1-s) / 2)}\right) \mathcal{D}^{-1}(-1)^{|i|+s}\left(\mathcal{T}_{\phi}^{-1}\right)^{*}\left(\Psi_{i}^{(s / 2)}\right)
\end{aligned}
$$

for positive-grade additional symmetries, and:

$$
\begin{aligned}
& \widetilde{\mathcal{M}}_{\overline{\mathcal{A}}}^{(-\ell / 2)} \equiv \delta_{\overline{\mathcal{A}}}^{(-\ell / 2)} \mathcal{T}_{\phi} \mathcal{T}_{\phi}^{-1}+\mathcal{T}_{\phi} \mathcal{M}_{\overline{\mathcal{A}}}^{(-\ell / 2)} \mathcal{T}_{\phi}^{-1}=\mathcal{T}_{\phi}\left(\mathcal{M}_{\overline{\mathcal{A}}}^{(-\ell / 2)}(\phi)-\delta_{\overline{\mathcal{A}}}^{(-\ell / 2)} \phi\right) \mathcal{D}^{-1} \phi^{-1}+ \\
& \quad+\sum_{I, J=1}^{2(N+r+1)} \overline{\mathcal{A}}_{I J}^{(-\ell / 2)} \sum_{s=0}^{\ell-1}(-1)^{s(|J|+\ell)} \mathcal{T}_{\phi}\left(\phi_{J}^{(-(\ell-1-s) / 2)}\right) \mathcal{D}^{-1}(-1)^{|I|+s}\left(\mathcal{T}_{\phi}^{-1}\right)^{*}\left(\psi_{I}^{(-s / 2)}\right) \\
& \widetilde{\mathcal{M}}_{\overline{\mathcal{F}}}^{(-\ell / 2)} \equiv \delta_{\overline{\mathcal{F}}}^{(-\ell / 2)} \mathcal{T}_{\phi} \mathcal{T}_{\phi}^{-1}-\mathcal{T}_{\phi} \mathcal{M}_{\overline{\mathcal{F}}}^{(-\ell / 2)} \mathcal{T}_{\phi}^{-1}=\mathcal{T}_{\phi}\left(\delta_{\overline{\mathcal{F}}}^{(-\ell / 2)} \phi-\mathcal{M}_{\overline{\mathcal{F}}}^{(-\ell / 2)}(\phi)\right) \mathcal{D}^{-1} \phi^{-1}+ \\
& \quad+\sum_{I, J=1}^{2(N+r+1)} \overline{\mathcal{F}}_{I J}^{(-\ell / 2)} \sum_{s=0}^{\ell-1}(-1)^{s(|J|+\ell)} \mathcal{T}_{\phi}\left(\phi_{J}^{(-(\ell-1-s) / 2)}\right) \mathcal{D}^{-1}(-1)^{|I|+s}\left(\mathcal{T}_{\phi}^{-1}\right)^{*}\left(\psi_{I}^{(-s / 2)}\right)
\end{aligned}
$$

for negative-grade additional symmetries (recall $R=2 r+1, M \equiv M_{B}+M_{F}=2 N+1$ ).

Now, we can repeat the same steps as in the analysis in the previous subsection 10.1 of the consistency of super-DB transformations with the bosonic and fermionic isospectral flows in order to find the conditions under which the (adjoint-)DB transformations (relations (222)- 223) and (224)(225)) preserve also the additional non-isospectral symmetries of $\operatorname{SKP}_{\left(R ; M_{B}, M_{F}\right)}$ integrable hierarchies. In other words, we have to find the conditions under which the super-pseudo-differential operators (251)-(254), generating the additional symmetries of the DB-transformed $S K P_{\left(R ; M_{B}, M_{F}\right)}$ hierarchy, can be represented in the same form as (66)-(67) and (171)-(172), respectively, with all pertinent (adjoint) super-eigenfunctions replaced with their (adjoint) DB-transformed counterparts. We obtain the following results for fermionic $\operatorname{SKP}_{\left(R ; M_{B}, M_{F}\right)}$ hierarchies (24) : 
- Super-DB transformations of the first type (i) $((228)-(230))$ preserve (up to an overall sign change of the fermionic flows) the following subalgebra of additional non-isospectral symmetries:

$$
\left(\widehat{G L}\left(M_{B}-1, M_{F}\right)\right)_{+} \oplus\left(\widehat{G L}^{\prime}(N+r+1, N+r+1)\right)_{-}
$$

- Super-DB transformations of the second type (ii) ((234)-(236)) preserve (up to an overall sign change of the fermionic flows) the following subalgebra of additional symmetries:

$$
\left(\widehat{G L}\left(M_{B}, M_{F}\right)\right)_{+} \oplus(\widehat{G L}(N+r, N+r+1))_{-}
$$

(here the positive-grade part includes the Manin-Radul isospectral flows).

For bosonic $\operatorname{SKP}_{\left(R ; M_{B}, M_{F}\right)}$ hierarchies (33) we obtain similar results with (255) and 256) replaced by:

$$
\left(\widehat{G L}_{M_{B}-1, M_{F}}\right)_{+} \oplus\left(\widehat{G L}_{N+r, N+r}^{\prime}\right)_{-}
$$

and

$$
\left(\widehat{G L}_{M_{B}, M_{F}}\right)_{+} \oplus\left(\widehat{G L}_{N+r-1, N+r}\right)_{-}
$$

respectively.

\subsection{Iterations of Superspace Darboux-Bäcklund Transformations and Wronskian-like Super- Determinant Solutions}

The general super-Darboux-Bäcklund orbit consists of successive applications of the allowed DB (217) and adjoint-DB (220) transformations as defined in subsection 10.1 (see Eqs.(222)-(239)). In particular, pairs of successive DB and adjoint-DB transformations are called binary DB transformations. Let us consider an iteration of $n$ successive binary DB transformations followed by $2 m$ successive DB transformations applied on arbitrary initial bosonic super-eigenfunction $\Phi$ :

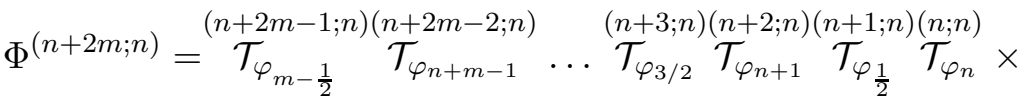

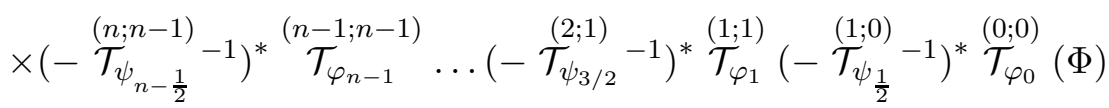

Recall that each (adjoint) DB transformation flips the Grassmann parity of the transformed object. Similarly, let us consider an iteration of $n$ successive binary DB transformations followed by $2 m+1$ successive DB transformations applied on arbitrary initial fermionic super-eigenfunction $F$ :

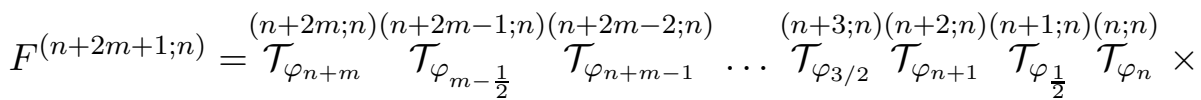

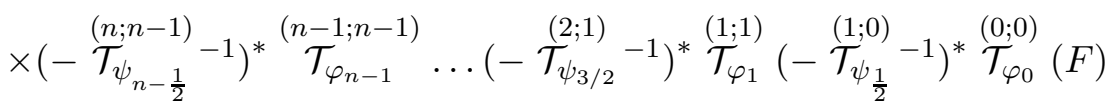

The upper indices $(k ; l)$ in (259)-(260) and below indicate iteration of (adjoint) DB transformations consisting of $k \mathrm{DB}$ steps and $l$ adjoint-DB steps. The objects entering each (adjoint) DB step in (259)-(260) are recurrsively defined as follows:

$$
\stackrel{(}{\mathcal{T}}_{\varphi_{k}}^{(k ; k)} \equiv \varphi_{k}^{(k ; k)} \mathcal{D} \frac{1}{\varphi_{k}^{(k ; k)}} \quad, \quad \stackrel{(}{\mathcal{T}}_{\psi_{k+\frac{1}{2}}}^{(k+1 ; k)} \equiv \psi_{k+\frac{1}{2}}^{(k+1 ; k)} \mathcal{D} \frac{1}{\psi_{k+\frac{1}{2}}^{(k+1 ; k)}}
$$




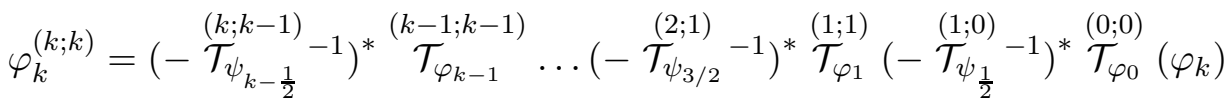

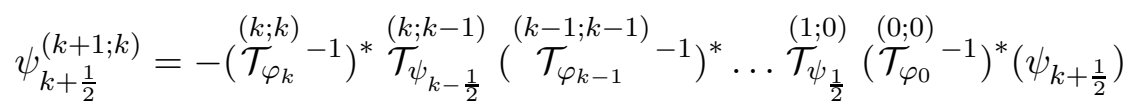

for $k=1, \ldots, n-1$, and:

$$
\begin{aligned}
& \stackrel{(n+2 l ; n)}{\mathcal{T}_{\varphi_{n+l}}} \equiv \varphi_{n+l}^{(n+2 l ; n)} \mathcal{D} \frac{1}{\varphi_{n+l}^{(n+2 l ; n)}} \quad, \quad \stackrel{(n+2 l+1 ; n)}{\mathcal{T}}_{\varphi_{l+\frac{1}{2}}} \equiv \varphi_{l+\frac{1}{2}}^{(n+2 l+1 ; n)} \mathcal{D} \frac{1}{\varphi_{l+\frac{1}{2}}^{(n+2 l+1 ; n)}}
\end{aligned}
$$

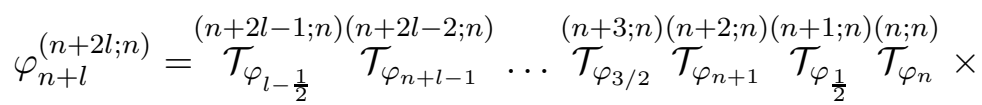

$$
\begin{aligned}
& \left.\times\left(-{\stackrel{(n ; n-1)}{\mathcal{T}} \psi_{n-\frac{1}{2}}}^{(-1}\right)^{*} \stackrel{(n-1 ; n-1)}{\mathcal{T}_{\varphi_{n-1}}} \ldots\left(-{\stackrel{\mathcal{T}}{\mathcal{T}_{3 / 2}}}_{\psi_{3}}^{(2 ; 1)}-1\right){\stackrel{(1 ; 1)}{\mathcal{T}_{\varphi_{1}}}}_{\left(-{\stackrel{\mathcal{T}}{\psi_{\frac{1}{2}}}}^{(1 ; 0)}-1\right.}\right)^{*} \stackrel{(0 ; 0)}{\mathcal{T}_{\varphi_{0}}}\left(\varphi_{n+l}\right)
\end{aligned}
$$

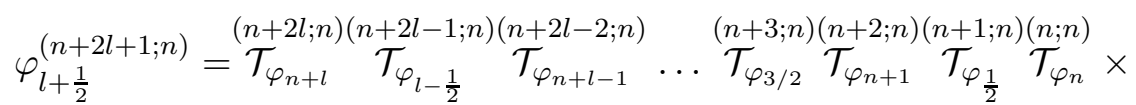

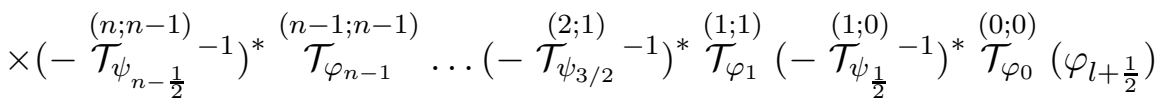

where $l=0,1, \ldots, m-1$. In (259)-(266) the sets $\left\{\varphi_{k}\right\}_{k=0}^{n+m}$ and $\left\{\varphi_{l-\frac{1}{2}}\right\}_{l=1}^{m}$ are bosonic/fermionic super-eigenfunctions, whereas $\left\{\psi_{k-\frac{1}{2}}\right\}_{k=1}^{n}$ are fermionic adjoint super-eigenfunctions. Let us also stress that DB-transformed superfunctions $F^{(n+2 m-1 ; n)}$ (260), $\varphi_{l+\frac{1}{2}}^{(n+2 l+1 ; n)}$ 266) and $\psi_{k+\frac{1}{2}}^{(k+1 ; k)}$ 263) are bosonic although the initial $F, \varphi_{l+\frac{1}{2}}$ and $\psi_{k+\frac{1}{2}}$ are fermionic.

During iteration of (adjoint) super-DB transformations we encounter Berezinians (super-determinants) whose matrix blocks possess the following special generalized Wronskian-like $k \times(m+n)$ matrix form:

$$
\begin{gathered}
\widetilde{W}_{k, m+n}^{(k ; n)}[\{\varphi\} \mid\{\psi\}] \equiv \widetilde{W}_{k, m+n}^{(k ; n)}\left[\varphi_{0}, \ldots, \varphi_{k-1} \mid \psi_{\frac{1}{2}}, \ldots, \psi_{n-\frac{1}{2}}\right]= \\
=\left(\begin{array}{cccc}
\varphi_{0} & \ldots & \cdots & \varphi_{k-1} \\
\vdots & \ddots & \ddots & \vdots \\
\partial^{m-1} \varphi_{0} & \cdots & \cdots & \partial^{m-1} \varphi_{k-1} \\
\mathcal{D}_{\theta}^{-1}\left(\varphi_{0} \psi_{\frac{1}{2}}\right) & \cdots & \cdots & \mathcal{D}_{\theta}^{-1}\left(\varphi_{k-1} \psi_{\frac{1}{2}}\right) \\
\vdots & \ddots & \ddots & \vdots \\
\mathcal{D}_{\theta}^{-1}\left(\varphi_{0} \psi_{n-\frac{1}{2}}\right) & \cdots & \cdots & \mathcal{D}_{\theta}^{-1}\left(\varphi_{k-1} \psi_{n-\frac{1}{2}}\right)
\end{array}\right)
\end{gathered}
$$

where $\{\varphi\} \equiv\left\{\varphi_{0}, \ldots, \varphi_{k-1}\right\}$ is a set of $k$ bosonic or fermionic superfunctions whereas $\{\psi\} \equiv$ $\left\{\psi_{\frac{1}{2}}, \ldots, \psi_{n-\frac{1}{2}}\right\}$ is a set of $n$ fermionic superfunctions. The generalized Wronskian-like matrix (267) is the supersymmetric generalization of the Wronskian-like block matrices entering the general Darboux-Bäcklund determinant solutions for the tau-functions of ordinary "bosonic" constrained KP hierarchies [26, 29]. In the special case of $n=0$ (267) reduces to the rectangular $k \times m$ Wronskian matrix:

$$
W_{k, m}\left[\varphi_{0}, \ldots, \varphi_{k-1}\right]=\left(\begin{array}{cccc}
\varphi_{0} & \cdots & \cdots & \varphi_{k-1} \\
\partial \varphi_{0} & \cdots & \cdots & \partial \varphi_{k-1} \\
\vdots & \ddots & \ddots & \vdots \\
\partial^{m-1} \varphi_{0} & \cdots & \cdots & \partial^{m-1} \varphi_{k-1}
\end{array}\right)
$$


In ref. 15] the explicit form of iterations of super-DB transformations not accompanied by adjoint-DB transformations, i.e., with $n=0$ in (259)-(260), has been derived:

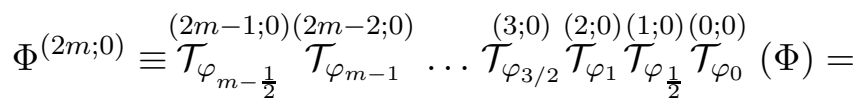

$$
\begin{aligned}
& =\frac{\operatorname{Ber}\left(\begin{array}{c|c}
W_{m+1, m+1}\left[\varphi_{0}, \ldots, \varphi_{m-1}, \Phi\right] & W_{m, m+1}\left[\varphi_{\frac{1}{2}}, \ldots, \varphi_{m-\frac{1}{2}}\right] \\
-------------- & ----------- \\
W_{m+1, m}\left[\mathcal{D}_{\theta} \varphi_{0}, \ldots, \mathcal{D}_{\theta} \varphi_{m-1}, \mathcal{D}_{\theta} \Phi\right] & W_{m, m}\left[\mathcal{D}_{\theta} \varphi_{\frac{1}{2}}, \ldots, \mathcal{D}_{\theta} \varphi_{m-\frac{1}{2}}\right]
\end{array}\right)}{\operatorname{Ber}\left(\begin{array}{c|c}
W_{m, m}\left[\varphi_{0}, \ldots, \varphi_{m-1}\right] & W_{m, m}\left[\varphi_{\frac{1}{2}}, \ldots, \varphi_{m-\frac{1}{2}}\right] \\
------------ & ----------------\mathcal{D}_{\theta} \\
W_{m, m}\left[\mathcal{D}_{\theta} \varphi_{0}, \ldots, \mathcal{D}_{\theta} \varphi_{m-1}\right] & W_{m, m}\left[\mathcal{D}_{\theta} \varphi_{\frac{1}{2}}, \ldots, \mathcal{D}_{\theta} \varphi_{m-\frac{1}{2}}\right]
\end{array}\right)} \\
& F^{(2 m+1 ; 0)} \equiv{\stackrel{(2 m ; 0)(2 m-1 ; 0)}{\mathcal{T}_{\varphi_{m}}}}_{\mathcal{T}_{\varphi_{n-\frac{1}{2}}}} \ldots{\stackrel{\mathcal{T}}{\mathcal{T}_{3 / 2}}}_{\mathcal{T}_{\varphi_{1}}}^{(3 ; 0)} \stackrel{(2 ; 0)}{\mathcal{T}_{\varphi_{1}}} \stackrel{(1 ; 0)}{\mathcal{T}}_{\varphi_{\frac{1}{2}}}^{(0 ; 0)} \mathcal{T}_{\varphi_{0}}(F)= \\
& =\frac{\operatorname{Ber}\left(\begin{array}{c|c}
W_{m+1, m+1}\left[\varphi_{0}, \ldots, \varphi_{m-1}, \varphi_{m}\right] & W_{m, m+1}\left[\varphi_{\frac{1}{2}}, \ldots, \varphi_{m-\frac{1}{2}}\right] \\
-------------- & ------------ \\
W_{m+1, m}\left[\mathcal{D}_{\theta} \varphi_{0}, \ldots, \mathcal{D}_{\theta} \varphi_{m-1}, \mathcal{D}_{\theta} \varphi_{m}\right] & W_{m, m}\left[\mathcal{D}_{\theta} \varphi_{\frac{1}{2}}, \ldots, \mathcal{D}_{\theta} \varphi_{m-\frac{1}{2}}\right]
\end{array}\right)}{\operatorname{Ber}\left(\begin{array}{c|c}
W_{m+1, m+1}\left[\varphi_{0}, \ldots, \varphi_{m}\right] & W_{m+1, m+1}\left[\varphi_{\frac{1}{2}}, \ldots, \varphi_{m-\frac{1}{2}}, F\right] \\
------------ & -------------------\mathcal{D}_{\theta} \\
W_{m+1, m+1}\left[\mathcal{D}_{\theta} \varphi_{0}, \ldots, \mathcal{D}_{\theta} \varphi_{m}\right] & W_{m+1, m+1}\left[\mathcal{D}_{\theta} \varphi_{\frac{1}{2}}, \ldots, \mathcal{D}_{\theta} \varphi_{m-\frac{1}{2}}, \mathcal{D}_{\theta} F\right]
\end{array}\right)}
\end{aligned}
$$

Following similar techniques as in ref. 15] we can similarly express the general (adjoint) DB iterations (259)-(260) in the form of ratios of Berezinians containing generalized Wronskian-like matrix blocks (267). The results are as follows:

$$
\Phi^{(n+2 m ; n)}=\frac{\operatorname{Ber}\left(\begin{array}{c|c}
\widetilde{W}_{n+m+1, n+m+1}^{(n+m+1 ; n)}[\{\varphi\}, \Phi \mid\{\psi\}] & \widetilde{W}_{m, n+m+1}^{(m ; n)}\left[\left\{\varphi_{\left(\frac{1}{2}\right)}\right\} \mid\{\psi\}\right] \\
-------------- & ---------- \\
W_{n+m+1, m}\left[\mathcal{D}_{\theta} \varphi_{0}, \ldots, \mathcal{D}_{\theta} \varphi_{n+m-1}, \mathcal{D}_{\theta} \Phi\right] & W_{m, m}\left[\mathcal{D}_{\theta} \varphi_{\frac{1}{2}}, \ldots, \mathcal{D}_{\theta} \varphi_{m-\frac{1}{2}}\right]
\end{array}\right)}{\operatorname{Ber}\left(\begin{array}{c|c}
\widetilde{W}_{n+m, n+m}^{(n+m ; m)}[\{\varphi\} \mid\{\psi\}] & \widetilde{W}_{m, n+m}^{(m ; n)}\left[\left\{\varphi_{\left(\frac{1}{2}\right)}\right\} \mid\{\psi\}\right] \\
------------- & ----------- \\
W_{n+m, m}\left[\mathcal{D}_{\theta} \varphi_{0}, \ldots, \mathcal{D}_{\theta} \varphi_{n+m-1}\right] & W_{m, m}\left[\mathcal{D}_{\theta} \varphi_{\frac{1}{2}}, \ldots, \mathcal{D}_{\theta} \varphi_{m-\frac{1}{2}}\right]
\end{array}\right)}
$$

$$
\begin{gathered}
F^{(n+2 m+1 ; n)}=(-1)^{n} \times \\
\operatorname{Ber}\left(\begin{array}{c:c}
\widetilde{W}_{n+m+1, n+m+1}^{(n+m+1 ; n)}\left[\{\varphi\}, \varphi_{n+m} \mid\{\psi\}\right] & \widetilde{W}_{m, n+m+1}^{(m ; n)}\left[\left\{\varphi_{\left(\frac{1}{2}\right)}\right\} \mid\{\psi\}\right] \\
---------------- & ----------- \\
W_{n+m+1, m}\left[\mathcal{D}_{\theta} \varphi_{0}, \ldots, \mathcal{D}_{\theta} \varphi_{n+m-1}, \mathcal{D}_{\theta} \varphi_{n+m}\right] & W_{m, m}\left[\mathcal{D}_{\theta} \varphi_{\frac{1}{2}}, \ldots, \mathcal{D}_{\theta} \varphi_{m-\frac{1}{2}}\right]
\end{array}\right) \\
\hline \operatorname{Ber}\left(\begin{array}{c:c}
\widetilde{W}_{n+m+1, n+m+1}^{(n+m+1 ; m)}\left[\{\varphi\}, \varphi_{n+m} \mid\{\psi\}\right] & \widetilde{W}_{m+1, n+m+1}^{(m+1 ; n)}\left[\left\{\varphi_{\left(\frac{1}{2}\right)}\right\}, F \mid\{\psi\}\right] \\
-------------- & -------------- \\
W_{n+m+1, m+1}\left[\mathcal{D}_{\theta} \varphi_{0}, \ldots, \mathcal{D}_{\theta} \varphi_{n+m}\right] & W_{m+1, m+1}\left[\mathcal{D}_{\theta} \varphi_{\frac{1}{2}}, \ldots, \mathcal{D}_{\theta} \varphi_{m-\frac{1}{2}}, \mathcal{D}_{\theta} F\right]
\end{array}\right)
\end{gathered}
$$


with the notations:

$$
\{\varphi\} \equiv\left\{\varphi_{0}, \ldots, \varphi_{n+m-1}\right\} \quad, \quad\left\{\varphi_{\left(\frac{1}{2}\right)}\right\} \equiv\left\{\varphi_{\frac{1}{2}}, \ldots, \varphi_{m-\frac{1}{2}}\right\} \quad, \quad\{\psi\} \equiv\left\{\psi_{\frac{1}{2}}, \ldots, \psi_{n-\frac{1}{2}}\right\}
$$

As above, $\{\varphi\}$ and $\left\{\varphi_{\left(\frac{1}{2}\right)}\right\}$ are sets of bosonic/fermionic super-eigenfunctions whereas $\{\psi\}$ is a set of fermionic adjoint super-eigenfunctions of the constrained super-KP hierarchy $\operatorname{SKP}_{\left(R ; M_{B}, M_{F}\right)}(24)$. Let us recall that all pertinent (adjoint) super-eigenfunctions are of the form (54)-(53).

While calculating super-tau functions on the general super-DB orbit we will also need the following iteration of (adjoint) DB transformations on fermionic adjoint super-eigenfunctions $\Psi_{F}$ :

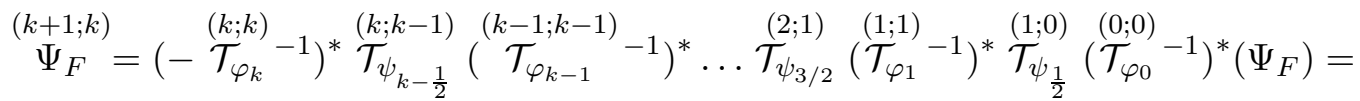

$$
\begin{aligned}
& \begin{array}{r||cccc}
\operatorname{det} \| & \mathcal{D}_{\theta}^{-1}\left(\varphi_{0} \psi_{\frac{1}{2}}\right) & \cdots & \mathcal{D}_{\theta}^{-1}\left(\varphi_{k-1} \psi_{\frac{1}{2}}\right) & \mathcal{D}_{\theta}^{-1}\left(\varphi_{k} \psi_{\frac{1}{2}}\right) \\
\vdots & \ddots & \vdots & \vdots \\
\mathcal{D}_{\theta}^{-1}\left(\varphi_{0} \psi_{k-\frac{1}{2}}\right) & \cdots & \mathcal{D}_{\theta}^{-1}\left(\varphi_{k-1} \psi_{k-\frac{1}{2}}\right) & \mathcal{D}_{\theta}^{-1}\left(\varphi_{k} \psi_{k-\frac{1}{2}}\right) \\
\mathcal{D}_{\theta}^{-1}\left(\varphi_{0} \Psi_{F}\right) & \cdots & \mathcal{D}_{\theta}^{-1}\left(\varphi_{k-1} \Psi_{F}\right) & \mathcal{D}_{\theta}^{-1}\left(\varphi_{k} \Psi_{F}\right)
\end{array}
\end{aligned}
$$

where $k=0,1, \ldots, n-1$.

Now, using relations (249) and taking into account (271)-(272) and (274), we derive the explicit expressions for the super-tau functions of constrained super-KP hierarchies on the general super-DB orbit:

$$
\begin{gathered}
\frac{\tau^{(0 ; 0)}}{\tau^{(n+2 m ; n)}}=(-1)^{m n+n(n-1) / 2} \times \\
\operatorname{Ber}\left(\begin{array}{c|c}
\widetilde{W}_{n+m, n+m}^{(n+m ; m)}[\{\varphi\} \mid\{\psi\}] & \widetilde{W}_{m, n+m}^{(m ; n)}\left[\left\{\varphi_{\left(\frac{1}{2}\right)}\right\} \mid\{\psi\}\right] \\
------------- & ----------- \\
W_{n+m, m}\left[\mathcal{D}_{\theta} \varphi_{0}, \ldots, \mathcal{D}_{\theta} \varphi_{n+m-1}\right] & W_{m, m}\left[\mathcal{D}_{\theta} \varphi_{\frac{1}{2}}, \ldots, \mathcal{D}_{\theta} \varphi_{m-\frac{1}{2}}\right]
\end{array}\right) \\
\operatorname{Ber}\left(\begin{array}{c|c}
\tau^{(n+2 m+1 ; n)} \tau^{(0 ; 0)}=(-1)^{m n+n(n-1) / 2} \times \\
---------------- & ---------- \\
W_{n+m+1, m}\left[\mathcal{D}_{\theta} \varphi_{0}, \ldots, \mathcal{D}_{\theta} \varphi_{n+m-1}, \mathcal{D}_{\theta} \varphi_{n+m}\right] & W_{m, m}\left[\mathcal{D}_{\theta} \varphi_{\frac{1}{2}}, \ldots, \mathcal{D}_{\theta} \varphi_{m-\frac{1}{2}}\right]
\end{array}\right) \\
\left(\begin{array}{c}
\widetilde{W}_{n+m+1, n+m+1}^{(n+m+1 ; n)}\left[\{\varphi\}, \varphi_{n+m} \mid\{\psi\}\right] \\
W_{m, n+m+1}\left[\left\{\varphi_{\left(\frac{1}{2}\right)}\right\} \mid\{\psi\}\right]
\end{array}\right.
\end{gathered}
$$

where again notaions (273) have been used.

\subsection{Examples: "Super-Soliton" Solutions}


Now, let us write down some explicit examples of Wronskian-like Berezinian solutions for the superspace tau-function 275)-276). We shall consider the simplest case of a constrained superKP hierarchy - the $S K P_{(1 ; 1,0)}$ hierarchy defined by the super-Lax operator:

$$
\mathcal{L} \equiv \mathcal{L}_{(1 ; 1,0)}=\mathcal{D}+f_{0}+\Phi \mathcal{D}^{-1} \Psi
$$

where $\Phi, \Psi$ are bosonic (adjoint) super-eigenfunctions. We take the initial $\tau^{(0 ; 0)}=$ const, i.e., the initial super-Lax operator is the "free" one $\mathcal{L}^{(0 ; 0)} \equiv \mathcal{L}_{\frac{1}{2}, \frac{1}{2}}^{(0 ; 0)}=\mathcal{D}$. The initial "free" supereigenfunction $\Phi^{(0 ; 0)} \equiv \Phi_{0}$ satisfies according to 27 :

$$
\begin{aligned}
\frac{\partial}{\partial t_{k}} \Phi_{0} & =\partial_{x}^{k} \Phi_{0} \quad, \quad \mathcal{D}_{n} \Phi_{0}=-\mathcal{D}_{\theta}^{2 n-1} \Phi_{0} \\
\Phi_{0}(t, \theta) & =\int d \lambda\left[\varphi_{B}(\lambda)+\left(\theta-\sum_{n \geq 1} \lambda^{n-1} \theta_{n}\right) \varphi_{F}(\lambda)\right] e^{\sum_{l \geq 1} \lambda^{l}\left(t_{l}+\theta \theta_{l}\right)}
\end{aligned}
$$

where $\varphi_{B}(\lambda), \varphi_{F}(\lambda)$ are arbitrary bosonic (fermionic) "spectral" densities.

Let us consider iterations of pure DB transformations (i.e., no mixed binary DB transformations). For the simplest $S K P_{(1 ; 1,0)}$ case this means substituting in the Berezinian expressions (269)-(270):

$$
\varphi_{k}=\partial_{x}^{k-1} \Phi_{0}, \varphi_{k-\frac{1}{2}}=\mathcal{D}_{\theta}^{k} \Phi_{0} \text { for } k=0,1, \ldots, m
$$

It is easy to show [14, 15] that in this case (269)-(270) reduce to the following ratios of ordinary Wronskian determinants:

$$
\begin{gathered}
\tau^{(2 m ; 0)=} \frac{\mathcal{W}_{m}\left[\partial_{x} \Phi_{0}, \ldots, \partial_{x}^{m} \Phi_{0}\right]}{\mathcal{W}_{m}\left[\Phi_{0}, \ldots, \partial_{x}^{(m-1)} \Phi_{0}\right]} \quad, \quad \tau^{(2 m+1 ; 0)}=\frac{\mathcal{W}_{m+1}\left[\Phi_{0}, \ldots, \partial_{x}^{m} \Phi_{0}\right]}{\mathcal{W}_{m}\left[\partial_{x} \Phi_{0}, \ldots, \partial_{x}^{m} \Phi_{0}\right]} \\
\mathcal{W}_{m}\left[\varphi_{0}, \ldots, \varphi_{m-1}\right] \equiv \operatorname{det} W_{m, m}\left[\varphi_{0}, \ldots, \varphi_{m-1}\right]
\end{gathered}
$$

where $\Phi_{0}$ is given by (279). In particular, choosing for the bosonic (fermionic) "spectral" densities in Eq. 279) $\varphi_{B}(\lambda)=\sum_{i=1}^{N} c_{i} \delta\left(\lambda-\lambda_{i}\right), \varphi_{F}(\lambda)=\sum_{i=1}^{N} \epsilon_{i} \delta\left(\lambda-\lambda_{i}\right)$, where $c_{i}, \lambda_{i}$ and $\epsilon_{i}$ are Grassmanneven and Grassmann-odd constants, respectively, we have for $\Phi_{0}$ :

$$
\Phi_{0}=\sum_{i=1}^{N}\left[c_{i}+\left(\theta-\sum_{n \geq 1} \lambda_{i}^{n-1} \theta_{n}\right) \epsilon_{i}\right] e^{\sum_{l \geq 1} \lambda_{i}^{l}\left(t_{l}+\theta \theta_{l}\right)}
$$

Substituting (282) into (281) we obtain the following "super-soliton" solutions for the super-tau function of simplest constrained super-KP hierarchy $\operatorname{SKP}_{(1 ; 1,0)}(277)$ :

$$
\begin{aligned}
& \tau^{(2 m+1 ; 0)}=\frac{\sum_{1 \leq i_{1}<\ldots<i_{m+1} \leq N}\left(\begin{array}{c}
N \\
m+1
\end{array}\right) \widetilde{c}_{i_{1}} \ldots \widetilde{c}_{i_{m+1}} E_{i_{1}} \ldots E_{i_{m+1}} \Delta_{m+1}^{2}\left(\lambda_{i_{1}}, \ldots, \lambda_{i_{m+1}}\right)}{\sum_{1 \leq j_{1}<\ldots<j_{m} \leq N}\left(\begin{array}{c}
N \\
m
\end{array}\right) \widetilde{c}_{j_{1}} \ldots \widetilde{c}_{j_{m}} E_{j_{1}} \ldots E_{j_{m}} \lambda_{j_{1}} \ldots \lambda_{j_{m}} \Delta_{m}^{2}\left(\lambda_{j_{1}}, \ldots, \lambda_{j_{m}}\right)} \\
& \widetilde{c}_{i} \equiv c_{i}+\left(\theta-\sum_{n \geq 1} \lambda_{i}^{n-1} \theta_{n}\right) \epsilon_{i} \quad, \quad E_{i} \equiv e^{\sum_{l \geq 1} \lambda_{i}^{l}\left(t_{l}+\theta \theta_{l}\right)} \\
& \Delta_{m}\left(\lambda_{i_{1}}, \ldots, \lambda_{i_{m}}\right) \equiv \operatorname{det}\left\|\lambda_{i_{a}}^{b-1}\right\|_{a, b=1, \ldots, m}
\end{aligned}
$$




\section{Outlook}

In the present paper we have provided a systematic derivation of the full algebra of additional non-isospectral symmetries of constrained (reduced) supersymmetric KP hierarchies of integrable ("super-soliton") nonlinear evolution equations in $N=1$ superspace, which turns out to be a semi-direct product of Virasoro algebra with a superloop superalgebra of the form given in (189)-(190) above. We also explicitly constructed the superspace analogues of (constrained) multicomponent KP hierarchies where the multi-component set of Manin-Radul-type isospectral evolution "times" can be viewed as special subsets of additional symmetry non-isospectral flows of ordinary one-component supersymmetric KP hierarchies. We also showed that the (constrained) multicomponent supersymmetric KP hierarchies contain the supersymmetric generalization of DaveyStewartson higher-dimensional nonlinear evolution equations. We studied in detail the conditions for (adjoint) super-Darboux-Bäcklund transformations to preserve both Manin-Radul isospectral flows as well as the algebra of additional non-isospectral symmeries of constrained super-KP hierarchies, and we presented the explicit Darboux-Bäcklund solutions for the pertinent super-tau functions ("super-soliton" solutions).

The results of the present work suggest a number of interesting problems for further research:

- Systematic study of the supersymmetric extended hierarchies (multi-component constrained supersymmetric KP hierarchies) introduced in Section 7 above, which are obtained from scalar (one-component) $S K P_{\left(R ; M_{B}, M_{F}\right)}$ hierarchies enhanced by Manin-Radul-like subsets of additional symmetry flows - of both "positive" and "negative" grades. This implies providing an explicit super-Lax and superspace tau-function description of the Manin-Radul-like subsets of additional symmetry flows, as it has been done in ref. 27, 29] for the ordinary "bosonic" case.

- Revealing other physically interesting nonlinear systems contained within the multi-component constrained supersymmetric KP hierarchies besides the supersymmetric Davey-Stewartson system (Section 7 ), such as supersymmetric extensions of the $N$-wave resonant wave system, supersymmetric Toda lattice etc.

- Systematic reformulation of the results of the present paper about additional non-isospectral symmetries, obtained in the framework of Sato super-pseudo-differential operator formalism, within the supersymmetric generalization of the Drinfeld-Sokolov algebraic "dressing" approach (for initial steps in this direction, see ref.[36])

- Systematic study of the physical properties and significance of the new very broad class of super-soliton-like solutions obtained in Section 10 above.

Acknowledgements We gratefully acknowledge support from U.S. National Science Foundation grant INT-9724747. We thank Prof. H. Aratyn and the Physics Department of University of Illinois at Chicago for hospitality during NATO Advanced Workshop on "Integrable Hierarchies and Modern Physical Theories" (July 2000) where the results of the present paper were first reported. We are grateful to H. Aratyn for collaboration in the initial stage of the project. This work is also partially supported by Bulgarian NSF grant F-904/99.

\section{References}


[1] L. Alvarez-Gaumé, H. Itoyama, J. Mañes and A. Zadra, Int. J. Mod. Phys. A7 (1992) 5337; L. Alvarez-Gaumé, K. Becker, M. Becker, R. Emperan and J. Mañes, Int. J. Mod. Phys. A8 (1993) 2297

[2] S. Stanciu, Commun. Math. Phys. 165 (1994) 261, hep-th/9407189;

J.M. Figueroa-O'Farrill and S. Stanciu, Phys. Lett. 316B (1993) 282

[3] M. Chaichain and P. Kulish, Phys. Lett. 78B (1978) 413;

P. Di Vecchia and S. Ferrara, Nucl. Phys. B130 (1977) 93

[4] Yu. Manin and A. Radul, Commun. Math. Phys. 98 (1985) 65

[5] K. Ueno and H. Yamada, Adv. Studies in Pure Math., 16 (1988) 373-426;

K. Ueno, H. Yamada and K. Ikeda, Commun. Math. Phys. 124 (1989) 57

[6] V. Kac and J. van de Leur, Ann. Inst. Fourier 37 (1987) 99;

V. Kac and E. Medina, Letters in Math. Phys. 37 (1996) 435;

A. LeClair, Nucl. Phys. B314 (1989) 425;

M. Mulase, J. Diff. Geom. 34 (1991) 651;

J. Rabin, Commun. Math. Phys. 137 (1991) 533

[7] L. Martinez Alonso and E. Medina Reus, J. Math. Phys. 36 (1995) 4898;

A. Ibort, L. Martinez Alonso and E. Medina Reus, J. Math. Phys. 37 (1996) 6157

[8] P. Mathieu, J. Math. Phys. 29 (1988) 2499;

S. Belluci, E. Ivanov, S. Krivonos and A. Pichugin, Phys. Lett. 312B (1993) 463 hepth/9305078);

F. Delduc, E. Ivanov and S. Krivonos, J. Math. Phys. 37 (1996) 1356 hep-th/9510033);

F. Toppan, Int. J. Mod. Phys. A11 (1996) 3257 (hep-th/9506133)

[9] Z. Popowicz, J. Physics A29 (1996) 1281 (hep-th/9510185);

J. C. Brunelli and A. Das, Phys. Lett. 337B (1994) 303 (hep-th/9406214);

L. Bonora, S. Krivonos and A. Sorin, Nucl. Phys. B477 (1996) 835 (hep-th/9604165);

F. Delduc and L. Gallot, Commun. Math. Phys. 190 (1997) 395 (solv-int/9609008);

J.-C. Shaw and M.-H. Tu, Mod. Phys. Lett. A13 (1998) 979 (solv-int/9805002);

Z. Popowicz, Phys. Lett. 459B (1999) 150 (hep-th/9903198)

[10] Q.P. Liu and M. Mañas, in Lecture Notes in Physics, vol. 502, Springer (1998) (solvint/9711002)

[11] F. Delduc, L. Gallot and A. Sorin, Nucl. Phys. B558 (1999) 545 (solv-int/9907004);

A. Leznov and A. Sorin, Nucl. Phys. (Proc. Suppl.) B56 (1997) 258

[12] H. Aratyn and C. Rasinariu, Phys. Lett. 391B (1997) 99 (hep-th/9608107);

H. Aratyn, A. Das and C. Rasinariu, Mod. Phys. Lett. A12 (1997) 2623 (solv-int/9704119)

[13] H. Aratyn, A. Das, C. Rasinariu and A.H. Zimerman, in "Supersymmetry and Integrable Models", H. Aratyn et.al. (Eds.), Springer-Verlag, 1998 (Lecture Notes in Physics 502)

[14] H. Aratyn, E. Nissimov and S. Pacheva, J. Math. Phys. 40 (1999) 2922 solv-int/9801021)

[15] H. Aratyn, E. Nissimov and S. Pacheva, "Topics in Theoretical Phys.", vol. II, pp. 17-24, H. Aratyn, L.A. Ferreira, J.F. Gomes (eds.), IFT-São Paulo, SP-1998 (solv-int/9808004) 
[16] O. Lechtenfeld and A. Sorin, Nucl. Phys. B566 (2000) 489 (solv-int/9907021);

F. Delduc and A. Sorin, Nucl. Phys. B577 (2000) 461 (solv-int/9911005);

O. Lechtenfeld and A. Sorin, solv-int/9912010, nlin.SI/0007009;

V. Kadyshevsky and A. Sorin, nlin.SI/0011009, to appear in "Integrable Hierarchies and Modern Physical Theories", NATO Science Series, H. Aratyn ed., Kluwer Acad. Publ.

[17] W. Oevel and B. Fuchsteiner, Phys. Lett. 88A (1982) 323;

B. Fuchsteiner, J. Math. Phys. 34 (1993) 514 (and references therein);

H. H. Chen, Y. C. Lee and J. E. Lin, Physica D9 (1983) 439

[18] A. Orlov and E. Schulman, Letters in Math. Phys. 12 (1986) 171;

P. Grinevich and A. Orlov, in "Problems of Modern Quantum Field Theory", A. Belavin et.al. (eds.), Springer (1989);

A. Orlov, in Nonlinear Processes in Physics: Proceedings of the III Potsdam-V Kiev Workshop, A. Fokas et.al. (eds.), Springer (1993)

[19] P. van Moerbeke, in "Lectures on Integrable Systems", eds. O. Babelon et.al., World Scientific (1994);

L. Dickey, Acta Applicandae Math. 47 (1997) 243

[20] D. Chen and H. Zhang, J. Physics A24 (1991) 377;

V.E. Adler, Theor. Math. Phys. 89 (1991) 323;

S. Aoyama and Y. Kodama, Phys. Lett. 278B (1992) 56; Phys. Lett. 295B (1992) 190;

T.J. Hollowood, J.L. Miramontes and J. J. Sánchez Guillén, J. Physics A27 (1994) 4629 (hepth/9311067);

M. Mañas, J. Geom. Phys. 17 (1995) 1

[21] H. Aratyn, E. Nissimov and S. Pacheva, Int. J. Mod. Phys. A12 (1997) 1265 (hep-th/9607234);

H. Aratyn, E. Nissimov and S. Pacheva, Phys. Lett. 228A (1997) 164 (hep-th/9602068)

[22] L. Dickey, Commun. Math. Phys. 167 (1995) 227 (hep-th/9312015);

M. Adler, T. Shiota and P. van Moerbeke, Commun. Math. Phys. 171 (1995) 547

[23] A. Orlov and P. Winternitz, Theor. Math. Phys. 113 (1997) 1393 solv-int/9701008)

[24] A. Orlov and D. Scherbin, nlin.SI/0001001

[25] H. Aratyn, E. Nissimov and S. Pacheva, Phys. Lett. 244A (1998) 245 (solv-int/9712012);

H. Aratyn, E. Nissimov and S. Pacheva, "Topics in Theoretical Phys.", vol. II, pp. 25-33, H. Aratyn, L.A. Ferreira, J.F. Gomes (eds.), IFT-São Paulo, SP-1998 solv-int/9808003)

[26] H. Aratyn, E. Nissimov and S. Pacheva, in "Bäcklund and Darboux Transformations: The Geometry of Soliton Theory", eds. P. Winternitz et.al., AMS Press, 2001 (solv-int/9904024)

[27] H. Aratyn, J.F. Gomes, E. Nissimov and S. Pacheva, Loop-Algebra and Virasoro Symmetries of Integrable Hierarchies of KP Type, in Applicable Analysis, special issue dedicated to R. Carroll's 70th birthday, Ch.Q. Bu ed., nlin.SI/0004040.

[28] E. Date, M. Jimbo, M. Kashiwara and T. Miwa, Transformation Groups for Soliton Equations. III. Operator Approach to the Kadomtsev-Petviashvili Equation, J. Phys. Soc. Japan 50 (1981) 3806 ; 
K. Ueno and K. Takasaki, Toda Lattice Hierarchy, in in Adv. Stud. Pure Math., vol. 4, NorthHolland, 1984, pp. 1-95;

V. Kac and J. van de Leur, The n-Component KP Hierarchy and Representation Theory, in Important Developments in Soliton Theory, A. Fokas and V.E. Zakharov (eds.), Springer (1993) (hep-th/9308137)

[29] E. Nissimov and S. Pacheva, Int. J. Mod. Phys. A16 (2001) (to appear) (hep-th/0009194)

[30] H. Aratyn, L.A. Ferreira, J.F. Gomes and A.H. Zimerman, J. Physics A33 (2000) L331 (nlin.SI/0007002)

[31] B. Konopelchenko and W. Strampp, J. Math. Phys. 33 (1992) 3676;

Y. Cheng, J. Math. Phys. 33 (1992) 3774;

B. Xu and Y. Li, J. Physics A25 (1992) 2957;

J. Sidorenko and W. Strampp, J. Math. Phys. 34 (1993) 1429;

W. Oevel and W. Strampp, Commun. Math. Phys. 157 (1993) 1;

Y. Cheng, W. Strampp and B. Zhang, Commun. Math. Phys. 168 (1995) 117

[32] W. Oevel, Physica A195 (1993) 533;

W. Oevel and W. Schief, Rev. Math. Phys. 6 (1994) 1301

[33] H. Aratyn, E. Nissimov and S. Pacheva, Phys. Lett. 314B (1993) 41 (hep-th/9306035);

H. Aratyn, E. Nissimov and S. Pacheva, Phys. Lett. 331B (1994) 82 (hep-th/9401058);

F. Yu, Letters in Math. Phys. 29 (1993) 175 hep-th/9301053);

H. Aratyn, E. Nissimov, S. Pacheva and A.H. Zimerman, Int. J. Mod. Phys. A10 (1995) 2537 (hep-th/9407117);

H. Aratyn, J. Gomes and A. Zimmerman, J. Math. Phys. 36 (1995) 3419 (hep-th/9408104)

[34] L. Bonora and C.S. Xiong, Int. J. Mod. Phys. A8 (1993) 2973 (hep-th/9209041); Nucl. Phys. B405 (1993) 191 (hep-th/9212070); J. Math. Phys. 35 (1994) 5781 (hep-th/9311070)

[35] F. Delduc and L. Gallot, solv-int/9802013;

J. Madsen and J. Miramontes, hep-th/9905103

[36] H. Aratyn, J.F. Gomes, E. Nissimov, S. Pacheva and A. Zimerman, nlin.SI/0012042, to appear in "Integrable Hierarchies and Modern Physical Theories", NATO Science Series, H. Aratyn ed., Kluwer Acad. Publ.

[37] J. Harnad and B. Kupershmidt, Commun. Math. Phys. 132 (1990) 315

[38] A. Das, E. Sezgin and S.J. Sin, Phys. Lett. 227B (1992) 435 (hep-th/9111054);

M. Manas, L. Martinez Alonso and E. Medina, Physica D87 (1995) 105

[39] H. Aratyn, E. Nissimov and S. Pacheva, Commun. Math. Phys. 193 (1998) 493 (solvint/9701017)

[40] L. Dickey, "Soliton Equations and Hamiltonian Systems", World Scientific (1991)

[41] F. Delduc, F. Gieres, S. Gourmelen and S. Theisen, Int. J. Mod. Phys. A14 (1999) 4043

[42] H. Aratyn, in Lecture Notes in Physics, vol. 502, Springer (1998);

H. Aratyn, J. Geom. Phys. 30 (1999) 295, and references therein 